\title{
A possible functional link between RNA degradation and transcription in Bacillus subtilis
}

\author{
Dissertation \\ for the award of the degree \\ "Doctor of Philosophy" \\ Division of Mathematics and Natural Sciences \\ of the Georg-August-University Göttingen \\ within the program "Microbiology and Biochemistry" \\ of the Georg-August University School of Science (GAUSS)
}

submitted by

Martin Benda

from Prague (Czech Republic)

Göttingen 2020 



\section{Thesis Committee}

Prof. Dr. Jörg Stülke (Supervisor and $1^{\text {st }}$ Reviewer)

Institute for Microbiology and Genetics, Department of General Microbiology, Georg-August-University Göttingen

Prof. Dr. Rolf Daniel (2 ${ }^{\text {nd }}$ Reviewer)

Institute for Microbiology and Genetics, Department of Genomic and Applied Microbiology, Georg-AugustUniversity Göttingen

Prof. Dr. Fabian M. Commichau

Institute for Biotechnology, Department of Synthetic Microbiology, Brandenburg University of Technology Cottbus-Senftenberg

\section{Additional members of the Examination Board}

Prof. Dr. Markus Bohnsack

Department of Molecular Biology, University Medical Center Göttingen

Prof. Dr. Patrick Cramer

Department of Molecular Biology, Max Planck Institute for Biophysical Chemistry, Göttingen

Prof. Dr. Stefanie Pöggeler

Institute for Microbiology and Genetics, Department of Genetics of Eukaryotic Microorganisms, GeorgAugust-University Göttingen

Date of oral examination: September $17^{\text {th }}, 2020$ 
I hereby declare that this Ph.D. thesis entitled "A possible functional link between RNA degradation and transcription in Bacillus subtilis" has been written independently and with no other sources and aids than quoted.

Martin Benda 


\section{Acknowledgements}

First of all, I would like to express my deep thanks to my supervisor Prof. Dr. Jörg Stülke for the great guidance during this work. He put his full trust in me and gave me the opportunity to work on this intricate, but engaging topic of RNA degradation. I could always turn to him with questions, I have been encouraged in moments of despair and supported in developing my own scientific ideas.

My immense thanks go to Prof. Dr. Fabian Commichau and Prof. Dr. Rolf Daniel for being members of my Thesis Advisory Committee and for broadening our view of the topic with meaningful comments and ideas. I would also like to thank Dr. Katrin Gunka who introduced me both to the project and to the lab and made the transition to a new working environment easier for me. I thank G2L Göttingen and especially Dr. Anja Poehlein for all the sequencing performed and discussion of the results. Many thanks go to Julia Busse and Gabriele Beyer for their help with the experimental work. Importantly, huge thanks go to Silvia Carillo-Castellón, all the work on this thesis would have been much more difficult without a constant supply of sterile tips, tubes or freshly prepared media.

I would also like to thank my students Simon Wölfel, Jonas Jennrich, Leon Daniau, Melin Güzel, Fabian Fiedler and Maxim Wintergoller, not only for their contribution to this thesis, but mainly for the great time I had working with all of them. I learned many new skills during that time and realized, quite unexpectedly, how much I like teaching.

My huge thanks go to all former and current members of the Stülke, Commichau and Rismondo labs I have met here. There were many of you and yet everyone kind, helpful in the laboratory and creating warm and friendly atmosphere not only in the laboratory but also outside.

I would also like to express my gratitude to Dr. Libor Krásný and all members of his lab. I learned the essential basics of laboratory and scientific work during my undergraduate time there and when this doctoral thesis turned its way towards his expertise, I received warm and great support again.

Finally and above all, I would like to thank my wife Klára for the daily unconditional support in all aspects of life. She always stands by my side and supports me, even if it meant moving abroad to a completely new environment and brought inconvenience to her own career. Without such a great support, I would have never been able to complete this thesis. Big thanks go also to my parents and my whole family, they have always been there for me since my first steps and only thanks to that I got up all the way to this thesis. 



\section{Table of contents}

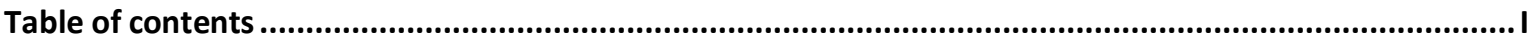

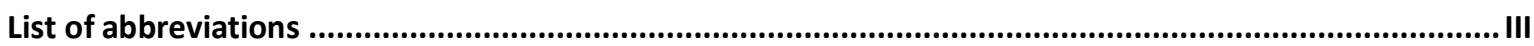

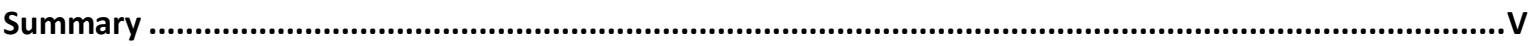

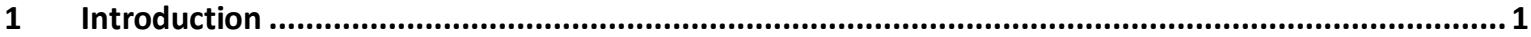

1.1 mRNA degradation and RNA degradosomes in bacteria................................................... 2

1.2 mRNA degradation and degradosome-like network of $B$. subtilis ..................................... 6

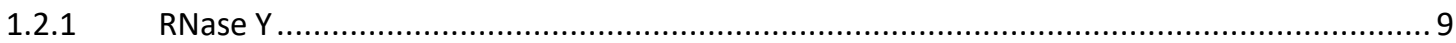

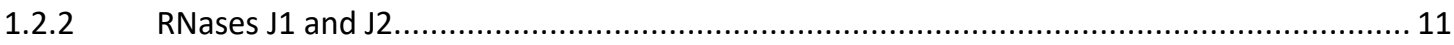

1.2.3 Polynucleotide phosphorylase (PNPase) ..................................................... 13

1.2.4 CshA, a DEAD-box RNA helicase ..................................................................... 14

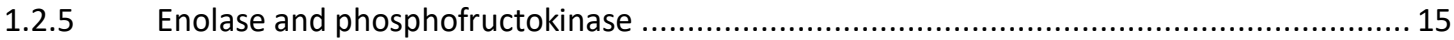

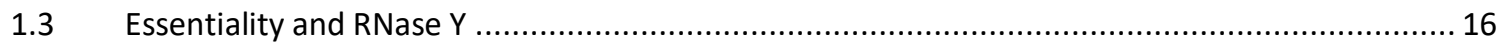

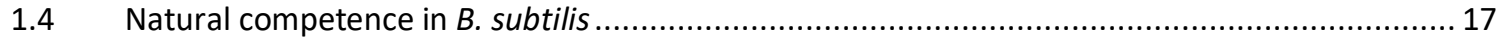

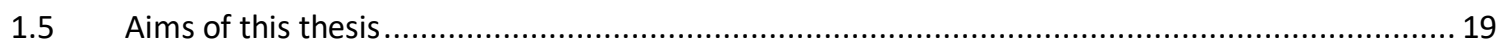

2 Quasi-essentiality of RNase $Y$ in Bacillus subtilis is caused by its critical role in the control of mRNA

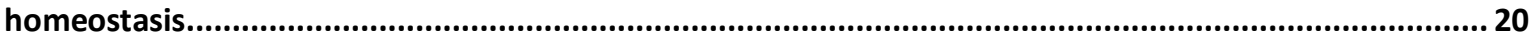

3 The YtrBCDEF ABC transporter is involved in the control of social activities in Bacillus subtilis ........ 46

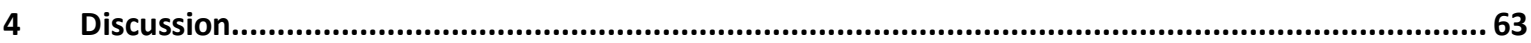

4.1 Suppressor mutant screen revealed initiation of bulk mRNA degradation as the pivotal function of RNase $Y .$.

4.2 Analysis of the rny suppressor mutants brings new insights into the regulation of the RNA

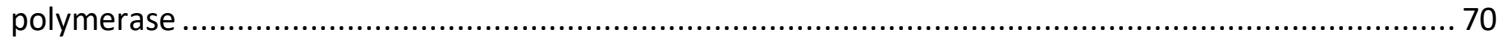

4.3 Loss of RNase Y leads to phenotypic effects independent of the total mRNA accumulation........ 73

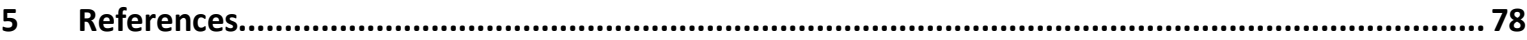

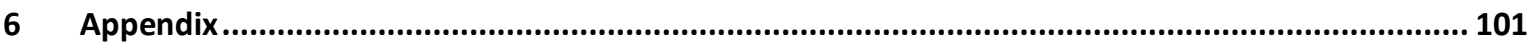

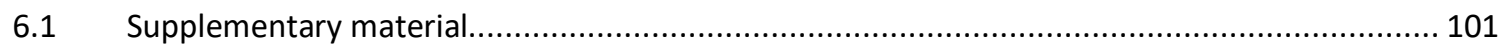

6.2 Bacterial strains, plasmids and oligonucleotides .................................................. 110

7 Curriculum Vitae. 


\section{List of abbreviations}

\section{General}

\begin{tabular}{|c|c|c|c|}
\hline A & adenosine & NTD & $\mathrm{N}$-terminal domain \\
\hline$A B C$ & ATP-binding cassette & OD & optical density \\
\hline Aco & aconitase & Orn & oligoribonuclease \\
\hline AMP & adenosine monophosphate & $\mathbf{P}$ & phosphate \\
\hline aphA3 & kanamycin resistance gene & $\mathbf{P}$ & promoter \\
\hline Asn & asparagine & PNPase & polynucleotide phosphorylase \\
\hline ATP & adenosine triphosphate & PCR & polymerase chain reaction \\
\hline B. & Bacillus & PFK & phosphofructokinase \\
\hline C & cytosine & $\mathrm{pH}$ & power of hydrogen \\
\hline c. & Caulobacter & RBS & ribosomal binding site \\
\hline cat & chloramphenicol resistance gene & rev & reverse \\
\hline CTD & C-terminal domain & RNA & ribonucleic acid \\
\hline DLN & degradosome-like network & RNase & ribonuclease \\
\hline DNA & deoxyribonucleic acid & rRNA & ribosomal RNA \\
\hline E. & Escherichia & S. & Staphylococcus \\
\hline e. $g$. & exempli gratia (for example) & S. & Streptococcus \\
\hline Eno & enolase & spc & spectinomycin resistance gene \\
\hline ermC & erythromycin resistance gene & SRNA & small RNA \\
\hline et al. & et alia (and others) & SRP & signal recognition particle \\
\hline FDR & false discovery rate & $\mathbf{T}$ & thymine \\
\hline Fig. & figure & Term. & transcriptional terminator \\
\hline fwd & forward & tRNA & transfer RNA \\
\hline G & guanosine & Trp & tryptophan \\
\hline Glu & glutamic acid & Tyr & tyrosine \\
\hline$H$. & Helicobacter & vs. & versus \\
\hline i. e. & id est (that is) & $\Delta$ & deletion \\
\hline IPTG & isopropyl $\beta$-D-1-thiogalactopyranoside & & \\
\hline kan & kanamycin & \multirow{2}{*}{\multicolumn{2}{|c|}{ Prefixes }} \\
\hline LB & lysogeny broth & & \\
\hline Leu & leucine & $\mathbf{k}$ & kilo \\
\hline LFH & long flanking homology & $\mathbf{m}$ & milli \\
\hline mRNA & messenger RNA & $\mu$ & micro \\
\hline$n$ & nucleotide & $\mathbf{n}$ & nano \\
\hline
\end{tabular}




\section{Units}

$\begin{array}{ll}{ }^{\circ} \mathbf{C} & \text { degree Celsius } \\ \text { bp } & \text { base pair } \\ \mathbf{g} & \text { gram } \\ \mathbf{g} & \text { standard gravity } \\ \mathbf{h} & \text { hour } \\ \mathbf{~} & \text { liter } \\ \mathbf{m i n} & \text { minute } \\ \mathbf{m o l} & \text { mol } \\ \mathbf{M} & \text { molar } \\ \mathbf{S} & \text { second }\end{array}$




\section{Summary}

Cellular levels of RNA depend on the rate of its synthesis and degradation. While synthesis is performed by RNA polymerase conserved in all domains of life, the enzymes responsible for RNA degradation are more unique even among organisms from the same domain. In the best studied bacterium, the gram-negative Escherichia coli, RNA degradation is achieved through a protein complex called RNA degradosome, which is assembled around the essential endoribonuclease

RNase E. However, RNase E is not present in the gram-positive model organism Bacillus subtilis. Instead, an enzyme called RNase $Y$ (rny) has been proposed as its functional counterpart responsible for the initiation of RNA degradation. Nevertheless, unlike RNase E of E. coli, it can be deleted from the genome, leaving an open question of its true significance and function. This project was designed to get a deeper understanding of the crucial process of RNA degradation in B. subtilis and of the role RNase $Y$ plays there. Although RNase $Y$ is dispensable for survival, the rny gene deletion leads to detrimental phenotypic effects, including filamentous growth, impaired cellular morphology or defects in the development of genetic competence and sporulation. The rny mutant strain also lyses rapidly and subsequently suppressor colonies appear. Using this natural force of suppressor evolution, we could demonstrate that no other RNase can take over the tasks of RNase Y. Conversely, all identified mutations were aimed to reduce RNA synthesis. This was achieved either by inactivation of transcription factors in conjunction with duplication of core RNA polymerase genes, which results in decreased number of correctly assembled RNA polymerase complexes, or, if the first suppressing mechanism was prevented, by mutations occurring directly in the RNA polymerase core genes, leading to orders of magnitude decrease in transcription. The fact that the mutations always affect RNA synthesis, a process on the opposite side of RNA life to the one RNase $Y$ acts, suggest close collaboration of RNase $Y$ with the RNA polymerase in establishing stable equilibrium between RNA synthesis and degradation. While the suppressor mutant analysis helped to identify the pivotal function of RNase $Y$, it did not necessarily provide an explanation for all the phenotypes associated with the deletion of the rny gene. In an attempt to better understand such phenotypes, RNA-sequencing analysis revealed global remodeling of gene expression in the rny strain. Furthermore, a screening system to recognize the reasons for the loss of genetic competence was established and helped to decipher the reasons for the loss of competence in the rny mutant as well as in other strains, among them in the $y$ trA mutant overexpressing putative $A B C$ transporter YtrBCDEF. This was shown to act in remodeling of the cell wall thickness, which hampers development of genetic competence as well as other lifestyles of $B$. subtilis. The possible influence of a disordered cell wall is also discussed as a potential reason for the loss of competence in the rny mutant. 


\section{Introduction}

All organisms are dependent on their ability to adapt to the surrounding environments and to use the available resources for their survival and reproduction. Due to their small size, bacteria are extremely vulnerable to changing environmental conditions and are therefore equipped with remarkable abilities to accommodate to the changing and challenging conditions. These abilities include short generation time, fast evolution, rapid modulation of gene expression or differentiation into specific cell types.

Crucial for fast adaptation is to regulate the amount and/or activity of proteins. This could be done either directly on the protein level or indirectly by modulating levels of messenger RNA (mRNA). The cellular level of mRNA is determined by the rate of its synthesis and degradation. Synthesis of mRNA is performed by a multi-subunit enzyme called RNA polymerase in process of transcription, which is subject to strict control and regulation. However, this control has a delayed onset of action and therefore mRNA levels must be also controlled by its degradation. Degradation of mRNA is thus one of the main mechanisms by which protein synthesis is regulated in all domains of life, since timely degradation of no longer necessary mRNAs is important to save energetic costs of translation and to release ribonucleotides for new rounds of condition adjusted transcription.

In conjunction with short generation time and fast adaptation, also half-lives of bacterial mRNAs are short, ranging from seconds to tens of minutes, with majority of transcripts from model bacterial organism Escherichia coli and Bacillus subtilis having mRNA half-lives shorter than 8 minutes (Hambraeus et al., 2003; Bernstein et al., 2004).

The enzymes responsible for the RNA degradation are called ribonucleases (RNases) and can be divided into two main groups (endo- and exo-ribonucleases) based on their mode of action. Endoribonucleases cleave RNA internally, while exoribonucleases attack the RNA molecule from its $5^{\prime}$ or $3^{\prime}$ ends. Whereas some RNases do have a very narrow substrate specificity and act on a limited number of transcripts, others are responsible for a broad degradation of cellular mRNAs. Those ribonucleases are often localized into multi-enzyme complexes to achieve high degree of effective cooperation. Such protein complexes can be found in all domains of life, as exosomes in eukaryotes and archaea (Mitchell et al., 1997; Evguenieva-Hackenberg et al., 2014), or as so-called RNA degradosomes in bacteria. These complexes have already been found in many bacterial species and will be further described in the following chapter. 


\section{1 mRNA degradation and RNA degradosomes in bacteria}

Degradation of mRNA is generally a very fast process once it starts, so it is the initial cleavage event which determines the degradation rate (Laalami et al., 2014). In theory, RNA degradation could be initiated by three different ways, by exoribonucleolytic degradation from either the $3^{\prime}$ or the $5^{\prime}$ end of RNA molecule or by internal endoribonucleolytic cleavage. However, mRNAs are often equipped with protective structures to prevent premature and uncontrolled degradation. The $3^{\prime}$ ends are usually protected from the action of exoribonucleases by secondary stem loop structures, moreover degradation from the $3^{\prime}$ end would be energetically very inefficient process, since the degradation would proceed in opposite direction than translation, thus leading to creation of truncated proteins (Laalami et al., 2014). The $5^{\prime}$ ends are mainly protected by a triphosphate group, although there is an increasing evidence about presence of other $5^{\prime}$ end protecting molecules such as nicotinamide adenine dinucleotide (NAD) (Cahová et al., 2015; Frindert et al., 2018). Therefore, due to the above-mentioned protections, initiation by exoribonuclease accounts only for minority of transcripts and it is the endoribonucleolytic attack, which usually initiates the degradation pathway (Mohanty and Kushner, 2018).

The endoribonuclease responsible for the initial cleavage in the best studied model organism E. coli is called RNase E. This enzyme is capable to initiate RNA degradation by direct endoribonucleolytic cleavage of single stranded mRNAs protected both on the $5^{\prime}$ and $3^{\prime}$ ends; however, this is the case only for some transcripts. Activity of RNase E, although it is an endoribonuclease, is in fact also affected by the phosphorylation state of the $5^{\prime}$ end, as RNase $E$ was shown in vitro to preferentially cleave transcripts with monophosphorylated $5^{\prime}$ ends, which rarely occur in nature (Mackie, 1998). In order to overcome this problem, E. coli is equipped with an additional enzymatic activity that alters the phosphorylation state of the $5^{\prime}$ end and creates monophosphorylated RNA molecules, thus facilitating the initial cleavage by RNase E. We can therefore define two different pathways by which the degradation is initiated, the $5^{\prime}$ end dependent pathway and the $5^{\prime}$ end independent pathway (see Fig. 1).

In the first case, the $5^{\prime}$ end dependent pathway is initiated by cleavage of two phosphates from the $5^{\prime}$ end, which leads to creation of $5^{\prime}$ monophosphorylated RNA molecule. An enzyme called RppH was traditionally thought to be responsible for this dephosphorylation (Deana et al., 2008). However, recent studies suggested that the dephosphorylation is a sequential process and that $\mathrm{RppH}$ can efficiently catalyze only the second reaction from diphosphate to monophosphate, leaving a possibility that another, as yet undiscovered enzyme, may be involved in this pathway (Luciano et al., 2017). When a 5' monophosphorylated RNA molecule is created, the presence of the monophosphate group stimulates endoribonucleolytic activity of RNase $\mathrm{E}$, leading to creation 
of two fragments. The first fragment does no longer have a stem loop structure on the $3^{\prime}$ end and therefore could be easily degraded by $3^{\prime}$-to- $5^{\prime}$ directed exoribonucleases like polynucleotide phosphorylase (PNPase). The second fragment is, thanks to its monophosphorylated $5^{\prime}$ end, a great substrate for further cleavage by RNase E. The whole RNA is this way gradually degraded up to di-nucleotides, which are then degraded into the individual nucleotides reusable in new round of transcription by an enzyme called Oligoribonuclease (Orn) (Kim et al., 2019).

The second pathway, $5^{\prime}$ end independent or sometimes also called direct entry pathway, is initiated by cleavage by RNase E. In this case RNase E directly accesses and cleaves an internal site of the mRNA molecule independently from the phosphorylation state of its $5^{\prime}$ end. Although this pathway seemed to be less likely due to the in vitro preference of RNase E for $5^{\prime}$ monophosphorylated RNAs, in reality it was shown to be the major initiating pathway in vivo in E. coli (Mackie, 1998; Clarke et al., 2014). The endoribonucleolytic cleavage here results again in two fragments, the first one contains the original $5^{\prime}$ end, but does no longer have a stem loop structure on the $3^{\prime}$ end and therefore, as in the $5^{\prime}$ end dependent pathway, is accessible for degradation by $3^{\prime}-5^{\prime}$ directed exoribonucleases. The second fragment, on the other hand, still contains a stem loop structure on the $3^{\prime}$ end, but is monophosphorylated on its $5^{\prime}$ end and therefore more susceptible for further cleavage events by RNase E. The RNA molecule is this way again further fragmented until dinucleotides are produced and degraded by Orn (Kim et al., 2019).

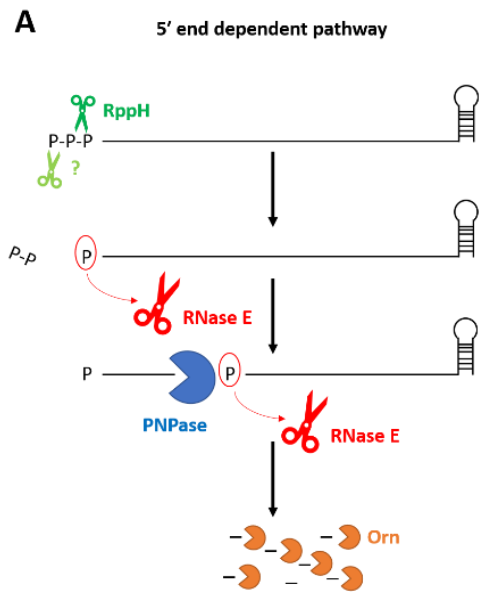

B 5' end independent pathway

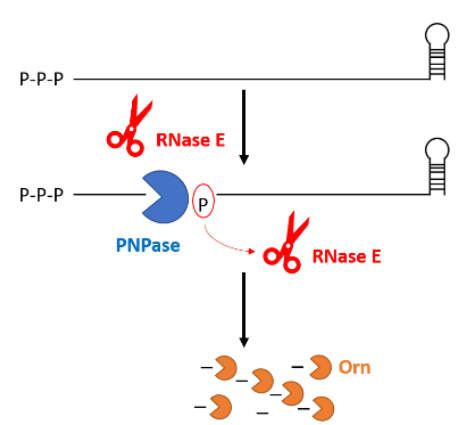

Figure 1: Schematic depiction of mRNA degradation pathways in E. coli

(A) In the $5^{\prime}$ end dependent pathway, pyrophosphate is first removed from the RNA molecule by RppH (dark green) and possible other enzyme (light green), monophosphorylated $5^{\prime}$ end activates RNase E (red), in further steps PNPase (blue) degrades RNA from the $3^{\prime}$ end. Finally, degradation of dinucleotides is achieved by Orn (orange). (B) In the $5^{\prime}$ end independent pathway, degradation is initiated directly by cleavage of RNase E, followed by actions of PNPase and Orn as described in A. 
As already mentioned, the enzymes involved in the degradation are often organized in complexes called RNA degradosomes. The enzymes present in the degradosomes as well as their amounts are varying between bacterial species. The only conserved requirement for the RNA degradosome is the presence of at least one RNase and one RNA helicase of the DEAD-box family, which supports the degradation by unwinding of complex RNA structures. Such a minimalistic two component degradosome could be found in the gastric pathogen Helicobacter pylori (Redko et al., 2013), however we can also find degradosomes with several components (for overview of some known bacterial degradosomes and their components see Table 1). The best studied degradosome is the one of the gram-negative model organism E. coli, where the core of this complex is composed of four proteins: RNase E, PNPase, RNA helicase RhIB and the glycolytic enzyme enolase.

Table 1: Comparison of proteins present in different bacterial RNA degradosomes.

Endoribonucleases are indicated with blue background, 5'-to-3' directed exoribonucleases with pink, 3'-to$5^{\prime}$ with orange, RNA helicases with green and metabolic enzymes with grey background. The table was constructed based on (Carpousis, 2007; Lehnik-Habrink et al., 2010; Hardwick et al., 2011; Redko et al., 2013; Płociński et al., 2019). Organisms are indicated as follows: E. coli = Escherichia coli, B. subtilis = Bacillus subtilis, M. tuberculosis = Mycobacterium tuberculosis, $C$. crescentus = Caulobacter crescentus, H. pylori = Helicobacter pylori.

\begin{tabular}{|lccccc|}
\hline & E. coli & B. subtilis & M. tuberculosis & C. crescentus & H. pylori \\
RNase E & $\checkmark$ & - & $\checkmark$ & $\checkmark$ & - \\
RNase Y & - & $\checkmark$ & $\checkmark$ & - & $\checkmark$ \\
RNase J & - & $\checkmark$ & $\checkmark$ & $\checkmark$ & - \\
PNPase & $\checkmark$ & $\checkmark$ & $\checkmark$ & $\checkmark$ \\
DEAD-box RNA helicase & $\checkmark$ & $\checkmark$ & - & Aco \\
Metabolic enzyme & Eno & Eno, PFK & - & $\checkmark$
\end{tabular}

The RNA degradosome of $E$. coli is assembled around the central essential ribonuclease RNase E (Carpousis, 2007). Whereas its N-terminal domain (NTD) contains the active center with endoribonuclease activity, important for initiation of mRNA degradation, the interactions to other degradosome components are mediated through the unstructured C-terminal domain (CTD). Furthermore, the CTD also contains an amphipathic helix through which is RNase E attached to the membrane (Khemici et al., 2008). Although the membrane localization of RNase $E$ and thus of the whole degradosome is not conserved among bacteria with RNase E homologues, and cytoplasmic degradosomes associated with the nucleoid were reported (Montero Llopis et al., 2010; Yan et al., 2020), it was recently shown to be important for precise regulation of RNA 
degradation in E. coli (Hadjeras et al., 2019). The detachment of RNase E from the membrane here leads to destabilization of the enzyme, slowdown of mRNA degradation, decreased growth rates as well as missing regulations by membrane associated proteins (Hadjeras et al., 2019).

Other degradosome components of $E$. coli are the polynucleotide phosphorylase (PNPase), which has 3'-to-5' exoribonucleolytic activity; the DEAD-box RNA helicase RhIB, which helps unwinding secondary structures in RNA and thus makes them accessible for the RNases; and the glycolytic enzyme enolase (Carpousis et al., 1994; Py et al., 1996; Miczak et al., 1996). The precise role of enolase in the complex is not fully understood, although there are reports suggesting that enolase is able to sense levels of glucose 6-phosphate and oxygen, respectively, to modulate RNase E action by promoting its disassociation from the membrane (Morita et al., 2004; Murashko and Lin-Chao, 2017).

In addition to those enzymes forming the core of the RNA degradosome complex, there are also other proteins associating with RNA degradosome only temporally or depending on conditions. For example, when RNA secondary structures are stabilized at low temperatures, the RNA degradosome can acquire additional DEAD-box RNA helicases to cope with an increased demand for resolving these structures to allow continuing RNA degradation, as shown not only for E. coli but also for Caulobacter crescentus (Prud'homme-Généreux et al., 2004; Khemici et al., 2004; Aguirre et al., 2017). Furthermore, Poly (A) polymerase I can associate with the degradosome to facilitate the RNA degradation, and RNA chaperone Hfq associates with the degradosome to aid in cleavage of sRNA tagged mRNA species (Carabetta et al., 2010; Bruce et al., 2018). Similarly, CspA and CspB, RNA binding cold shock proteins (Bae et al., 2000), were found to be associated to the degradosome complex in Mycobacterium tuberculosis (Płociński et al., 2019). In this organism, also the RNA polymerase can interact with the degradosome components, suggesting possible direct cooperation to establish the mRNA equilibrium (Płociński et al., 2019). Proteins RraA and RraB were further shown to interact with the degradosome to module its composition and activity (Lee et al., 2003; Gao et al., 2006) and also ribosomes were proposed to influence the degradosome activity by direct binding (Tsai et al., 2012; Redko et al., 2013). Many other proteins interact with the degradosome in a non-stoichiometric manner, for instance helicases SrmB and HrpA or RNase R of E. coli, however it is not clear whether these interactions do have a physiological role or whether they are just stochastic (Carabetta et al., 2010). Interestingly, association of the first and last enzymes of the degradation pathways ( $\mathrm{RppH}$ and Orn) was never observed. Since this thesis is focused on the model gram-positive organism Bacillus subtlis, the following parts will discuss more in depth mRNA degradation in this organism. 


\section{2 mRNA degradation and degradosome-like network of $B$. subtilis}

Due to the general importance of mRNA processing and degradation, it could be assumed that the key components are highly conserved among individual bacteria species. It was therefore surprising, that the gram-positive model organism B. subtilis does not contain any homolog of RNase $\mathrm{E}$, the central enzyme of mRNA degradation in E. coli. This also brought a question of whether there is an RNA degradosome in B. subtilis and if so, what does it look like?

This question was later addressed by the discovery of an enzyme called RNase $Y$ (Commichau et al., 2009; Shahbabian et al., 2009). Although RNase $Y$ does not have any sequence homology to RNase $E$ of $E$. coli, it was proposed to be the scaffolding protein of B. subtilis RNA degradosome based on interactions with other RNases, RNA helicase and glycolytic enzymes (Commichau et al., 2009; Lehnik-Habrink et al., 2010). Except these interactions, RNase $Y$ has also other striking functional similarities to RNase E of E. coli, since it also possesses endoribonuclease activity and is localized to the cytoplasmic membrane (Shahbabian et al., 2009; Cascante-Estepa et al., 2016). Apparently, the key players of the mRNA degradation process have evolved independently to fulfill very similar roles in the cells. This is further supported by the fact that the essential RNase E of $E$. coli could be substituted with RNase $Y$ of B. subtilis (Tamura et al., 2017).

The proposed RNA degradosome complex of $B$. subtilis built around central RNase $Y$ (see Fig. 2) is further composed of two other RNases showing endoribonuclease activity in vitro, the paralogues proteins RNases J1 and J2 (Even et al., 2005). In addition, those two RNases were also shown to have $5^{\prime}$-to- $3^{\prime}$ directed exoribonuclease activity, which is an activity completely missing in E. coli (Mathy et al., 2007). Furthermore, the proposed RNA degradosome contains $3^{\prime}$-to-5' directed exoribonuclease PNPase and a DEAD-box RNA helicase called CshA. Like the degradosome of $E$. coli, also this one contains the glycolytic enzyme enolase and on top of that another glycolytic enzyme, phosphofructokinase. Their role in the complex, however, remains mysterious.

In contrast to the RNA degradosome of $E$. coli, the degradosome of $B$. subtilis was never successfully purified as a complex and interactions between the individual components were only shown via bacterial-two hybrid studies or cross-linking pull down experiments (Coburn et al., 1999; Worrall et al., 2008; Commichau et al., 2009; Lehnik-Habrink et al., 2011a). In combination with data showing that the degradosome components localize mainly in the cytoplasm and do not co-localize with RNase $Y$ at the membrane (Cascante-Estepa et al., 2016), the existence of true degradosome in $B$. subtilis is questioned. Hence, recent literature is rather talking about degradosome-like network (DLN), since the interactions are probably just transient and highly dynamic (Durand and Condon, 2018). 


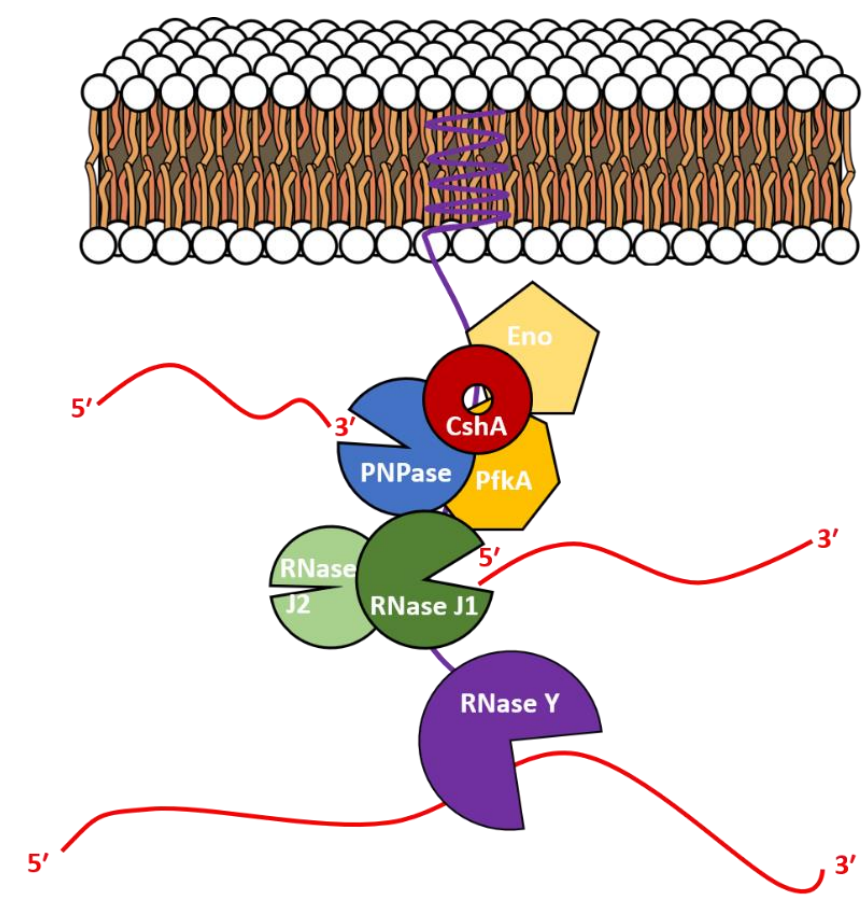

Figure 2: The proposed RNA degradosome complex of B. subtilis

The complex is anchored to the membrane through the N-terminus of RNase $\mathrm{Y}$, which also serves as a scaffold for the other components, complex of RNases J1/J2, PNPase, DEAD-box RNA helicase CshA and glycolytic enzymes enolase (Eno) and phosphofructokinase (PfkA). Modified from (Cho, 2017) and (Wölfel, 2018).

Initiation of mRNA degradation in $B$. subtilis can also occur by different pathways that are similar to those from E. coli (see Fig. 3). The $5^{\prime}$ end dependent pathway starts with dephosphorylation of RNA molecule by a phosphohydrolase also called $\mathrm{RppH}$, although this does not have a high degree of homology to the one from E. coli. RppH of B. subtilis can efficiently remove phosphates step by step as orthophosphates and thus, in contrast to $E$. coli, there is no need for additional enzymes (Richards et al., 2011). Nevertheless, there are reports about other enzymes capable of $5^{\prime}$ end dephosphorylation, which might be involved in this pathway as well (Frindert et al., 2019). The dephosphorylation step is followed either by complete exoribonucleolytic degradation of RNA by RNase $\mathrm{J} 1$ in $5^{\prime}$-to- $3^{\prime}$ direction (5' end dependent exopathway) or by endoribonucleolytic cleavage by RNase $Y$ ( $5^{\prime}$ end dependent endo-pathway), which has also preference for substrates with 5' monophosphates (Shahbabian et al., 2009; Richards et al., 2011). Fragments created by RNase $Y$ cleavage could be then rapidly degraded by action of exoribonucleases RNase J1 and PNPase. The final degradation step is not done by Orn enzyme as in E. coli, instead B. subtilis has at least two so-called nanoRNases encoded by the genes $n r n A$ and $n r n B$, which were shown to degrade short oligoribonucleotides up to $5 \mathrm{nt}$ long from the $3^{\prime}$ end. However, some capacity to complete the decay of RNA was also found in RNase J1 itself and 3'-to$5^{\prime}$ exoribonuclease YhaM, so it is possible that this function in B. subtilis is redundantly distributed among various enzymes (Mechold et al., 2007; Fang et al., 2009). 
Similarly to $E$. coli, B. subtilis can also initiate RNA degradation by a $5^{\prime}$ end independent pathway. Despite the fact that RNase $\mathrm{Y}$ has preference for $5^{\prime}$ monophosphorylated substrates, it was shown to efficiently initiate degradation of ermC mRNA regardless of the $5^{\prime}$ end phosphorylation state (Shahbabian et al., 2009; Yao et al., 2011). Taken together, the repertoire of degradation pathways is extended in the gram-positive model organism by the action of $5^{\prime}$-to$3^{\prime}$ directed exoribonuclease RNase J1.

A

5 ' end dependent exo-pathway

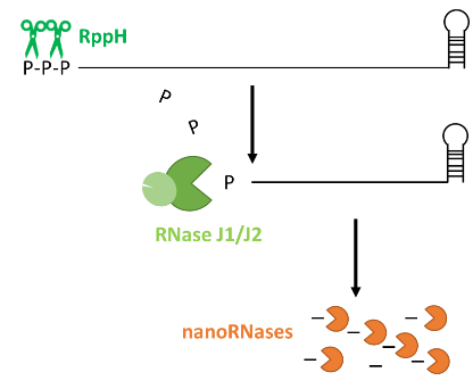

B

5 ' end dependent endo-pathway

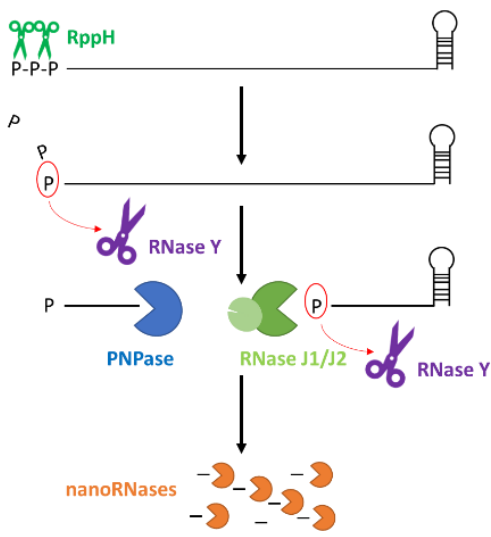

C

5 ' end independent pathway

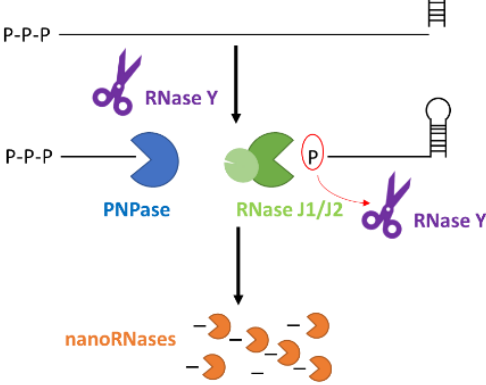

Figure 3: Schematic depiction of mRNA degradation pathways in B. subtilis

(A) In the $5^{\prime}$ end dependent exo-pathway, two orthophosphates are first removed from the RNA molecule by RppH (green scissors), monophosphorylated $5^{\prime}$ end activates RNases J1/J2 (green) to degrade the RNA exoribonucleolytically, followed by the degradation of short RNA fragments by nanoRNases (orange) (B) In the $5^{\prime}$ end dependent endo-pathway, RppH creates monophosphorylated $5^{\prime}$ end, which activates RNase $Y$ (purple scissors) for endoribonuclease cleavage, in further steps PNPase (blue) degrades RNA from the $3^{\prime}$ end and complex of the RNases $\mathrm{J} 1 / \mathrm{J} 2$ from the $5^{\prime}$ end. Finally, short RNA fragments are degraded by nanoRNAses. (C) In the $5^{\prime}$ end independent pathway, RNase $Y$ cleaves the transcript internally without a requirement for removal of phosphates from the $5^{\prime}$ end, this cleavage is followed by action of exoribonucleases as in B.

An obvious question which might appear is why there is no pathway initiating mRNA decay from the $3^{\prime}$ end? Although mRNAs are generally protected by stem loop structures at this terminus as already discussed, especially considering collaboration of the PNPase with RNA helicase present in the degradosome, this protective structure does not necessarily have to be a complete obstacle for such a pathway. Results obtained in previous studies, however, suggest that this is not the case, since absence of PNPase does not lead to strong global effect on gene expression and pnpA deletion strain accumulates only degradation fragments and not full length transcripts, as would be expected if PNPase is involved in the decay initiation (Luttinger et al., 1996; Oussenko et al., 2005). Therefore this possible initiation pathway seems to play only a minor role, if any, possibly in degradation of transcripts with Rho dependent terminators, which are rare in B. subtilis (Ingham et al., 1999; Liu et al., 2016). 


\subsubsection{RNase $Y$}

RNase $\mathrm{Y}$, encoded by the gene $r n y$, previously called $y m d A$, is the decay initiating enzyme and the scaffolding protein of the degradosome-like network (Commichau et al., 2009; Shahbabian et al., 2009). RNase $\mathrm{Y}$ is composed of four main domains, the $\mathrm{N}$-terminal domain which is responsible for anchoring of the enzyme to the membrane, an unstructured coiled-coil domain, which is likely a place for interactions with the other DLN components, the KH domain (ribonucleoprotein $\mathrm{K}$ homology), responsible for RNA binding, and the HD domain (His Asp), responsible for the endoribonucleolytic cleavage (Aravind and Koonin, 1998; Grishin, 2001; Shahbabian et al., 2009; Lehnik-Habrink et al., 2011a; Cho, 2017).

Except the interaction with other proteins, RNase $Y$ also interacts with itself and forms oligomers (Lehnik-Habrink et al., 2011a). Multimeric complexes of RNase $Y$ located in the membrane were recently spotted as dynamic foci using total internal reflection fluorescence microscopy (Hamouche et al., 2020). Those multimeric foci were proposed to contain less active form of the enzyme in absence of substrate (Hamouche et al., 2020), in contrast to the situation of RNase E of E. coli, where oligomers represent the more active form of the enzyme (Strahl et al., 2015).

The importance of the membrane localization of RNase $Y$ is not yet completely clear, it was initially shown that a membrane detached variant of RNase $Y$ is not able to complement for the membrane bound protein (Lehnik-Habrink et al., 2011a), however recent evidence suggests that membrane anchoring is not essential nor required for endoribonucleolytic activity. Its importance thus likely lays in spatial restriction of the enzymatic activity and/or in regulation of interactions with other proteins (Khemici et al., 2015; Hamouche et al., 2020).

As described above, RNase $Y$ participates in initiation of degradation of many transcripts, and in agreement with that, depletion of RNase $\mathrm{Y}$ led to stabilization and differential expression of huge amount of transcripts in three independent transcriptomic studies (Lehnik-Habrink et al., 2011b; Durand et al., 2012a; Laalami et al., 2013). Importantly, all those studies were performed with only a depletion of RNase $Y$, since by the time of their publication, the gene rny was thought to be essential.

Except its role in global degradation of mRNA, RNase $Y$ is also responsible for specific maturation events of functional RNAs, as shown for the RNA component of the RNAse $P$ ribozyme, scRNA or rnaC (Gilet et al., 2015; DeLoughery et al., 2018). RNase Y cleavage is also important for uncoupling expression of genes from some single operons, as it is the case for instance for infC-rpml-rplT, cggR-gapA-pgk-tpi-pgm-eno or glnR-glnA operons (Commichau et al., 2009; Bruscella et al., 2011; DeLoughery et al., 2018). 
As already mentioned, the rny gene was thought for a long time to be essential, however, in 2013 it was deleted by Figaro and coworkers and this was later reproduced in another study (Figaro et al., 2013; Koo et al., 2017). Nevertheless, deletion of rny gene leads to severe phenotypic defects. Colonies are small and smooth, quickly lysing and forming suppressor mutants (see Fig. 4). The doubling times are more than doubled as compared to the wild type, cell separation is impaired, so the rny mutant cells grow in chains (see Fig. 5). Furthermore, the strain is cold sensitive, its peptidoglycan layer is disordered, and also sporulation and development of genetic competence are abolished (Figaro et al., 2013).

A

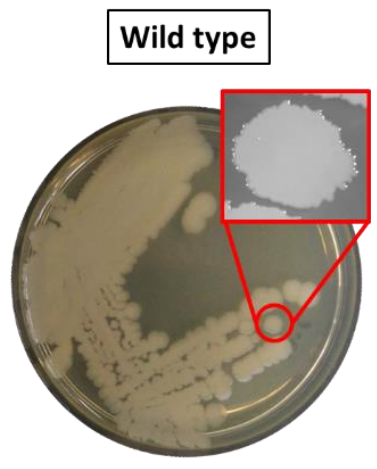

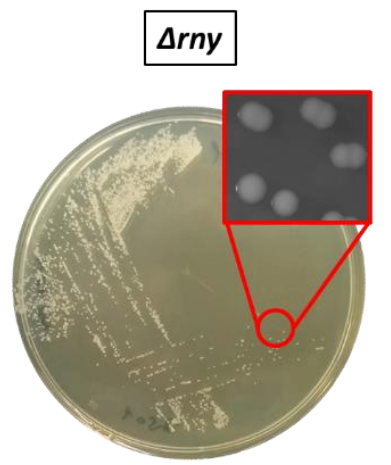

B

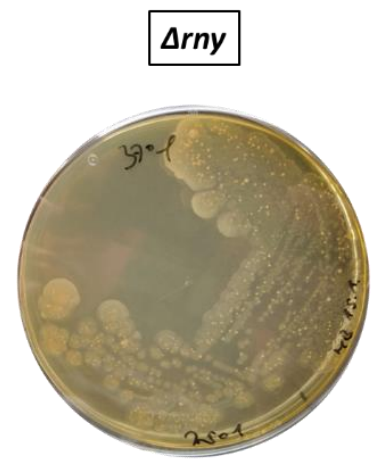

Figure 4: Colony morphology and suppressor formation of the rny mutant

(A) Comparison of colony morphology of wild type strain 168 and deletion mutant of rny gene. Plates were grown for 2 days $37^{\circ} \mathrm{C}$. All images were taken at the same magnification. (B) Suppressor mutants appear on the surface of lysing $\Delta r n y$ colonies. The picture was taken after 12 days of incubation at $37^{\circ} \mathrm{C}$.

RNase $Y$ is an endoribonuclease with a preference for $5^{\prime}$ monophosphorylated ends (Shahbabian et al., 2009). However, it is a matter of discussion, whether there is any sequence specificity for RNase Y cleavage events. In related organisms, preferential cleavage downstream of guanosine was reported both for Staphylococcus aureus and Streptococcus pyogenes (Khemici et al., 2015; Broglia et al., 2020). Furthermore, presence of double stranded secondary structure $6 \mathrm{nt}$ downstream of the cleavage site was reported to be decisive for cleavage of saePQRS operon mRNA in S. aureus (Marincola and Wolz, 2017). Concerning RNase Y from B. subtilis, no sequence preference for guanosine was identified so far, on the other hand presence of secondary structure might be the determinant also for the $B$. subtilis enzyme, as it was shown for Sadenosylmethionine riboswitches, where RNase $Y$ cleaves 6 nt downstream from the riboswitch aptamer structure (Shahbabian et al., 2009). Nevertheless, such a structural requirement was not identified in a whole transcriptome approach and might be specific only for certain transcripts (DeLoughery et al., 2018).

Except the proteins proposed to be part of the degradosome-like network, RNase $\mathrm{Y}$ also interacts with three additional proteins (YlbF, YmcA and YaaT) that form the so called Y-complex. 
This complex is necessary for RNase $Y$ cleavage (DeLoughery et al., 2016) and involved in the majority of known cleavage events. However, the phenotypes connected with the deletion of enzymes from this complex are far less severe than those of rny deletion, so the complex likely acts as a sort of specificity factor involved in some cleavage events. However, any sequence or other determinant of its action is yet to be discovered (DeLoughery et al., 2018). Although the mode of action of the $\mathrm{Y}$-complex is not clear, recent studies suggest that the complex modulates self-association of RNase $Y$ and thereby its activity (Hamouche et al., 2020).

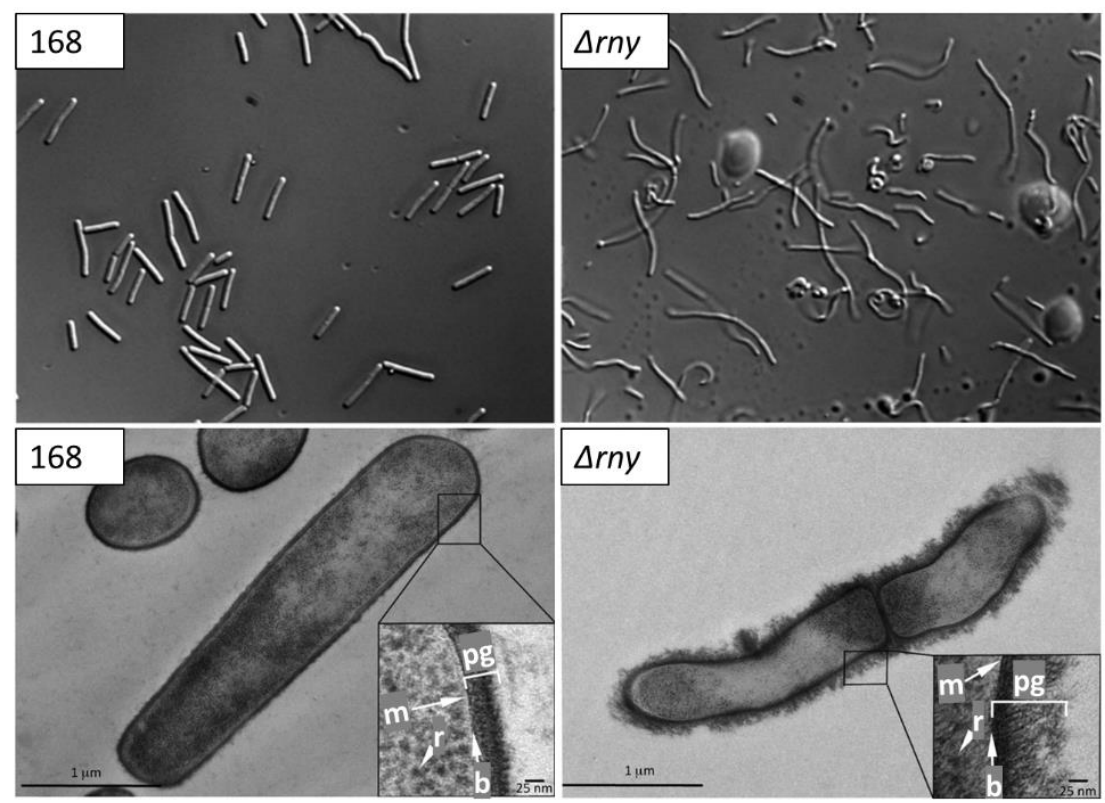

Figure 5: Phenotypic comparison of individual cells and their cell walls between wild type and $\Delta r n y$ The upper panel shows light microscopy images of wild type strain 168 (left) and $\Delta r n y$ cell morphology (right). The lower panel shows transmission electron microscopy of the altered cell wall of $\Delta r n y$ (right) comparing to wild type strain 168 (left). (pg) - peptidoglycan layer, (m) - cellular membrane, (r) ribosomes, (b) - base of the peptidoglycan layer. Modified from (Figaro et al., 2013).

\subsubsection{RNases J1 and J2}

RNases $\mathrm{J} 1$ and $\mathrm{J} 2$ (encoded by the genes $r n j A$ and $r n j B$ ) are paralogous proteins originally discovered during the search for possible functional homologs of RNase E in gram-positive bacteria thanks to their endoribonuclease activity in vitro (Even et al., 2005). However, later studies demonstrated that RNase J1 has unique bifunctional properties, since except the endoribonuclease activity it was also shown to degrade RNA exoribonucleolytically in $5^{\prime}$-to- $3^{\prime}$ direction. This is an activity that was at the time of the discovery thought to be absent from the bacterial domain of life (Mathy et al., 2007). Later on, the exoribonuclease activity was proposed to be the main one for RNase J1, based on the structural data showing that accommodation of a substrate for endoribonuclease cleavage into the active center is physically impossible without further conformational changes (Newman et al., 2011). 
After the discovery of RNase $J$ in B. subtlis, this enzyme was found to be conserved in different, mainly gram-positive bacterial species, but orthologues of RNase J could be also found in some archaea (Even et al., 2005; Clouet-d'Orval et al., 2018). This is striking since there are no homologs outside of bacteria for RNases $\mathrm{Y}$ and $\mathrm{E}$, the two degradation initiating enzymes in B. subtilis and E. coli, respectively.

Both RNases $\mathrm{J} 1$ and $\mathrm{J} 2$ are able to cleave substrates endoribonucleolytically in vitro with equal specificity and efficiency (Even et al., 2005), however the exoribonuclease activity of RNase J2 is about 100 times weaker than of RNase J1 (Mathy et al., 2010). That brings a question of RNase $\mathrm{J} 2$ relevance in vivo, especially since deletion of $r n j B$ gene does not lead to a significant phenotypic effect in B. subtilis. Since RNases J1 and J2 form a heterotetrametric complex in vivo (Mathy et al., 2010; Newman et al., 2011) it is possible that the main role of RNase J2 lays in altering cleavage site preferences of the $\mathrm{J} 1 / \mathrm{J} 2$ complex, which was shown to be different comparing to preferences of RNase J1 and RNase J2 alone (Mathy et al., 2010). The assumption that the ribonuclease activity is not the main role of RNase $\mathrm{J} 2$ is further supported by the fact that in S. aureus, where deletion of both genes for RNases J1 and J2 leads to strong phenotypic effects, only active site mutation of RNase $\mathrm{J} 1$ leads to the same phenotypes as deletion, whereas it is not the case for active site mutations of RNase J2 (Linder et al., 2014).

Similar to RNases $\mathrm{E}$ and $\mathrm{Y}$, activity of RNase $\mathrm{J} 1$ is also affected by the phosphorylation state of the $5^{\prime}$ end of its substrates, with preference for monophosphorylated RNAs (Mathy et al., 2007). RNase J1 is directly responsible for maturation of the $5^{\prime}$ end of 16S rRNA (Britton et al., 2007) and also for some specific cleavage events, as for instance cleavage of the yflS mRNA (Durand et al., 2017). It was also shown to participate in the turnover of the trp leader sequence and both maturation and degradation of $h b s$ mRNA (Deikus et al., 2008; Daou-Chabo et al., 2009; Deikus and Bechhofer, 2009). Although it is able to initiate mRNA degradation following $5^{\prime}$ end dephosphorylation (see Fig. 3), the global relevance of this pathway seems to be rather small, as assumed from non-altered global mRNA stability in double mutant lacking both RNases J1 and J2 (Even et al., 2005; Laalami et al., 2014). On the other hand, the role of RNase J1 in subsequent steps of mRNA degradation, following initial cleavage by RNase $Y$, seems to be crucial, since depletion of RNases J1 and J2 influences abundance of hundreds of transcripts (Mäder et al., 2008; Durand et al., 2012a).

Corresponding to its important role in RNA degradation, the rnjA gene was for a long time thought to be essential, and although it could be later deleted from the genome, its deletion leads to similar phenotypic effects as deletion of rny (Figaro et al., 2013). Thanks to the mutual interaction of RNase J1 with RNase Y, PNPase and phosphofructokinase (PFK), RNases J1 and J2 are proposed to be part of the degradosome-like network, although RNase J2 interacts only with 
RNase J1 (Commichau et al., 2009). Localization studies revealed that RNase J1 is mainly localized around the nucleoid (Cascante-Estepa et al., 2016), suggesting more pleiotropic role of RNase J1 in the cell than just being part of the degradosome-like network. Indeed, in agreement with the nucleoid localization, latest finding suggested its role in recovering of stalled RNA polymerases (Šiková et al., 2020).

\subsubsection{Polynucleotide phosphorylase (PNPase)}

PNPase is one of the four $3^{\prime}-$ to- $5^{\prime}$ exoribonucleases encoded in the genome of B. subtilis, together with RNase R, RNase PH and YhaM, and seems to be the most important one for the global mRNA degradation. This is based on the observation that accumulation of $5^{\prime}$ end precursors is not compensated by the other enzymes in a pnpA mutant (Oussenko et al., 2005; Liu et al., 2014). Furthermore, transcriptomic analysis showed that degradation of about $10 \%$ of transcripts is fully dependent on action of this 3'-to-5' exoribonuclease (Liu et al., 2014). Relevance of this enzyme for global mRNA degradation is even supported by the fact that PNPase was found to interact with other components of so-called degradosome-like network of B. subtilis (Commichau et al., 2009).

Unlike other components of the degradosome-like network, PNPase is widely conserved across bacterial species as well as eukaryotic organelles (Lin-Chao et al., 2007). Except its 3'-to-5' exoribonuclease activity, PNPase can also reverse the reaction and is able to polymerase RNA by addition of unspecific polyA tails on the $3^{\prime}$ ends of RNA molecules. In fact, this is the activity it was initially discovered for (Grunberg-Manago et al., 1956; Mohanty and Kushner, 2000).

Although PNPase is required for degradation of some specific transcripts, its activity was shown to be blocked by the presence of secondary structures on the RNA, which likely limits its role in the mRNA decay to downstream path after initial endoribonucleolytic cleavage (Farr et al., 1999). Initiation of mRNA degradation by PNPase itself is thus limited to few exceptional transcripts with Rho dependent terminators, as shown for s/rA mRNA (Liu et al., 2016). PNPase is also involved in maturation processes of some tRNAs (Bechhofer and Deutscher, 2019).

In addition to the role in RNA degradation, also other functions within the cell were proposed for PNPase, since PNPase can also degrade DNA molecules and the substrate specificity (DNA vs. RNA) is supposed to be determined by the energetic status of the cell. Furthermore PNPase is likely involved in double stranded break repair and homologous recombination processes, where its degradative and polymerizing activities are required to cooperate with RecN and RecA proteins (Cardenas et al., 2009; Cardenas et al., 2011). 
Deletion of pnpA gene is possible, however absence of PNPase leads to some phenotypic effects similar to those observed for the rny and rnjA mutants, i.e. strongly decreased transformation rates, growth in long filaments of cells, extremely poor growth at cold temperatures or increased sensitivity to tetracycline (Luttinger et al., 1996; Wang and Bechhofer, 1996; Figaro et al., 2013).

\subsubsection{CshA, a DEAD-box RNA helicase}

Another component of the degradosome-like network is a DEAD-box RNA helicase called CshA (cold shock helicase-like protein $\underline{A}$ ). This was initially described as a cold-shock response RNA helicase, since its expression seemed to be increased in low temperatures (Beckering et al., 2002; Hunger et al., 2006). However, later studies showed that cshA is expressed stably at different temperatures, media, as well as growth stages (Lehnik-Habrink et al., 2010; Nicolas et al., 2012).

Despite this condition independent expression, the role of CshA seems to be indeed more important at low temperatures under $22^{\circ} \mathrm{C}$, as could be judged from the impaired growth of the deletion mutant and curly phenotype reminiscent of the phenotpyes from mutants of other DLN components genes (for $\Delta r n y$, see Fig. 5) (Lehnik-Habrink et al., 2013; Figaro et al., 2013). The reason for the increased need for CshA during cold likely lies in the fact that under cold temperatures RNA secondary structures are more stable and therefore unwinding of these complex RNA structures is of higher importance.

DEAD-box helicases are in general composed of two RecA like domains consisting of 12 sequence motifs responsible for binding of ATP and RNA, respectively, and for subsequent remodeling of the RNA at the expanse of an ATP molecule (Linder and Jankowsky, 2011). Although most of the DEAD-box helicases are monomeric, CshA of B. subtilis forms a homodimer, which likely aids the enzyme to stay associated with the RNA molecule during multiple cycles of ATP hydrolysis. This can then result in an effective unwinding of RNA target providing substrate for action of RNA degrading enzymes, as it was shown for CshA of closely related organism Geobacillus stearothermophilus (Lehnik-Habrink et al., 2010; Huen et al., 2017).

CshA was proposed to be member of the DLN based on its interactions with RNase $Y$, PNPase, enolase and phosphofructokinase (Lehnik-Habrink et al., 2010). Except its general role in RNA degradation, CshA is also required for correct rRNA processing and thereby also ribosome biogenesis. Furthermore, deletion of cshA specifically affects expression of more than 200 genes (Lehnik-Habrink et al., 2013).

Interestingly, CshA was recently shown to be involved in activation of some alternative sigma factors. CshA is in the presence of glucose acetylated on two lysine residues and this 
acetylation seems to be crucial for $\sigma^{\mathrm{M}}$ and $\sigma^{\mathrm{X}}$ activation. Although the exact mechanisms is not known, this effect is independent from the presence of RNase $\mathrm{Y}$, which provides another evidence for a broader role of CshA in B. subtilis physiology (Ogura and Asai, 2016). This is even supported by the fact that CshA was also found to be associated with the RNA polymerase, where it could, for instance, stimulate expression from alternative sigma factor promoters (Delumeau et al., 2011).

In addition to CshA, other RNA helicases from the DEAD-box family are also present in the genome of $B$. subtilis. Despite the fact that these genes had been likely evolved by duplication, overexpression of the individual RNA helicases cannot complement for each other suggesting very specific role for each RNA helicase (González-Gutiérrez et al., 2018). Whether the other helicases except CshA also play a role in RNA degradation is not yet clear, however it is possible that one or more of them associates with the complex in condition dependent manner in analogy to similar situation in E. coli (Prud'homme-Généreux et al., 2004; Lehnik-Habrink et al., 2010).

\subsubsection{Enolase and phosphofructokinase}

The last two components of the degradosome-like network of $B$. subtilis are the glycolytic enzymes enolase (Eno) and phosphofructokinase (PFK), which were found both to interact with other DLN components as well as with each other (Commichau et al., 2009; Lehnik-Habrink et al., 2010; Lehnik-Habrink et al., 2011a; Newman et al., 2012). These two enzymes have a known role in glycolysis, where PFK phosphorylates fructose-6-phosphate to fructose-1,6-bisphosphate and enolase catalyzes conversion of 2-phosphoglycerate to phosphoenolpyruvate. In agreement with their main role outside of the RNA degradation, both are localized in the cytoplasm, with enolase aggregating at cell poles of some cells (Cascante-Estepa et al., 2016; El Najjar et al., 2018). Enolase is also part of the degradosome in E. coli and generally metabolic enzymes seem to be conserved among most of the RNA degradation machines (see 1.1). Nevertheless, the roles of metabolic enzymes in RNA degradation and specifically of Eno and PFK in the degradosome-like network of B. subtilis are rather unclear. Based on some initial studies about the role of enolase in the RNA degradosome of $E$. coli, it is likely that these enzymes can monitor the energetic status of the cell and adjust RNA degradation accordingly (Morita et al., 2004; Murashko and Lin-Chao, 2017). However, simple control of RNA degradation based on the energetic status of the cells would be much easier through direct binding of regulatory molecules (e.g. ATP, (p)ppGpp, c-di-AMP) to the RNA degrading enzymes, therefore the role of these glycolytic enzymes in the DLN is presumably more complex and will need further investigation in the future (Cho, 2017) 


\subsection{Essentiality and RNase $Y$}

Defining of the minimal necessary genetic equipment for sustainable and autonomous life on earth has long been one of the fundamental scientific topics. However, with the increased number of sequenced genomes it becomes more and more apparent that such a conserved set of essential genes does not exist even within one domain of life. Instead, essential functions seems to be more universal, but often performed by genes without any mutual sequence homology. Contradictory reports concerning essential genes have been published even about the same organisms, likely due to the slight difference between laboratory strains and/or conditions used for the screens (Lagesen et al., 2010; Martínez-Carranza et al., 2018).

It is also not easy to define what an essential gene actually is, because many genes might be essential under certain conditions, but dispensable under others. Despite that, several studies focusing on essentiality of $B$. subtilis genes have been performed. These were defined as genes that cannot be deleted from the genome to sustain laboratory growth at rich medium at $37^{\circ} \mathrm{C}$ (Kobayashi et al., 2003; Commichau et al., 2013). A recent whole genome study addressing gene essentiality exactly in these conditions identified 257 essential genes, SubtiWiki database currently defines even less essential genes in the genome of $B$. subtilis, specifically 251 protein coding and 2 sRNA coding (Koo et al., 2017; Zhu and Stülke, 2018). These numbers are however likely underestimated concerning minimal requirements for living cells, since they do not consider genes of redundant function and even the smallest autonomously replicating organism contains 473 genes (Hutchison et al., 2016).

RNase $E$ and RNase $Y$ of $E$. coli and B. subtilis, respectively, are in many aspects striking examples of convergent evolution, thanks to their similar structure, cellular localization and function. For a long time, it was thought that there is another similarity between these two enzymes, their essentiality, since any of the two genes could not be deleted from the genome in the respective studies (Kobayashi et al., 2003; Baba et al., 2006). However, in 2013 the rny gene was deleted from the chromosome of B. subtilis (Figaro et al., 2013) and this result was later reproduced by another independent study (Koo et al., 2017). Although this deletion leads to severe phenotypes as shown before, the rny gene is since then considered as non-essential.

This is a striking difference, since one might expect that initiation of mRNA degradation would be equally important and thus essential function in both model organisms. The difference might be most easily explained by the fact, that $B$. subtilis contains another ribonuclease RNase J1, which could also initiate some mRNA degradations events (see Fig. 3 ) in addition to RNase $Y$ and therefore initiation of mRNA degradation is not fully dependent on RNase $\mathrm{Y}$ in B. subtlis, whereas it is fully dependent on RNase E in E. coli. 
Regardless of the fact, that deletion of rny gene is possible, this leads to severe phenotypic defects and genomic instability (see Fig.4) suggesting that although not completely essential, it is inevitable for the rny strain to undergo further genetic adjustments for stable life. This is interestingly not the case for some even closely related organisms as Streptococcus pyogenes or Staphylococcus aureus (Marincola et al., 2012; Chen et al., 2013) bringing up an question, why is deletion of RNase $Y$ so harmful for $B$. subtilis. This has not yet been discovered and thus it remains possible that these phenotypes are caused because an essential cleavage event is missing, as found for instance for RNase III which is essential due to its cleavage of prophage encoded toxins (Durand et al., 2012b) or due to some general effect on total levels of multiple mRNA species.

\subsection{Natural competence in B. subtilis}

Loss of competence is not only a problem for the cellular survival in its natural habitat, but also major obstacle for the laboratory work. Since this thesis is focused on RNase $Y$ and the response of the cell to its absence, it is important to note that rny mutant strain has lost its ability to become competent (Figaro et al., 2013; Koo et al., 2017). That does not only bring a slowdown during the experimental work, but also a question why?

Competence of $B$. subtilis is evolved in a subpopulation of cells in response to increased cellular density and nutritional starvation. This is fully dependent on the levels of the master transcription regulator ComK (van Sinderen et al., 1995). Its expression is regulated in response to extra- and intra-cellular signals by various regulators on the level of gene expression, mRNA stability, as well as protein stability and only those cells, where ComK levels reach certain threshold become competent in an all or nothing scenario thanks to a ComK auto activation loop (Serror and Sonenshein, 1996; Turgay et al., 1998; Hoa et al., 2002; Hamoen et al., 2003b; Gamba et al., 2015).

There are various mechanism translating the signals into molecular responses. The cellular density is for instance sensed by the quorum sensing ComPA two component system, which can respond to the levels of the ComX pheromone (Weinrauch et al., 1990; Magnuson et al., 1994). Nutritional limitation is sensed by the transcription regulator CodY, which responds to levels of GTP and branched-chain amino acids (Serror and Sonenshein, 1996; Shivers and Sonenshein, 2004).

Interestingly, also other transcription regulators play a role in activation of competence (for instance SpoOA) and they are often shared between competence and development of other social behaviors in B. subtilis, like sporulation or biofilm formation (for review see (López et al., 
2009). When the master regulator ComK is present in sufficient amount, it activates expression of more than 100 genes responsible for the DNA uptake and the recombination itself (Berka et al., 2002; Hamoen et al., 2002; Ogura et al., 2002; Boonstra et al., 2020).

Not only absence of RNase $\mathrm{Y}$ leads to the loss of competence, there are many more genes whose deletion leads to the same phenotype (Koo et al., 2017). Reasons and mechanism for the loss of competence may be different. This can be a direct block of the DNA uptake or its further incorporation into the genome, as it is the case for deletion of the comGA and recA genes, respectively (Briley et al., 2011; Yadav et al., 2013). Alternatively, deletion of a gene can interfere with proper activation of the ComK master regulator. This is exactly the case for instance for the degU mutant, where absence of DegU blocks the competence development by dysregulating of comK expression (Shimane and Ogura, 2004). This is likely to be the case also in some of the uncharacterized competence mutants, since regulation of ComK is tightly controlled and finetuned on multiple levels and even small interferences with the regulation process might completely prevent development of genetic competence. Whether this is the case for loss of competence of rny mutant is to be discovered, however there is an indication that it could be, since comK expression is downregulated in the rny depletion strain (Lehnik-Habrink et al., 2011b; Laalami et al., 2013).

During transformation, DNA must pass some physical barriers such as the cell wall and the membrane. The gram-positive cell wall is known to be composed of a thick peptidoglycan layer, which consists of glycan chains cross-linked with peptides, and teichoic acids that can be attached either to the membrane (lipoteichoic acids) or to the peptidoglycan itself (wall teichoic acids). These passes through the top of the peptidoglycan and forms the uppermost layer of the cell wall (Silhavy et al., 2010). Interestingly, recent findings suggest that wall teichoic acids are specifically modified during development of genetic competence and that this is important for DNA binding, which could be blocked by the action of some wall teichoic acids targeting antibiotics (Mirouze et al., 2018). Furthermore, when the cell wall is too thick, DNA binding proteins might be masked by the peptidoglycan layer and thus be unable to efficiently bind DNA to the transport machinery. Since the rny mutant has indeed a thicker and disordered cell wall, these might be another reasons for the absence of competence. Lastly, it was also shown that DNA is preferentially bound to the cell poles, but the rny mutant grows in unseparated chains and cell poles are therefore not exposed to the environment, which might also prevent the DNA binding and transformation (Figaro et al., 2013; Mirouze et al., 2018). 


\subsection{Aims of this thesis}

Turnover of mRNA is a key regulatory process in all domains of life. RNase $\mathrm{Y}$ is the enzyme initiating this process in the well-studied model organism B. subtilis, yet it could be deleted from the genome and therefore is, by definition, considered not to be essential. However, such a deletion leads to severe phenotypes affecting many cellular processes and to high genetic instability. In the presented work the essentiality of RNase $Y$ and reasons for the deleterious phenotypes are addressed.

Analysis of suppressor mutants is used to identify the maintenance of equilibrium between RNA synthesis and degradation as the quasi-essential function missing in the rny mutant. Furthermore, speed of evolutionary forces and natural selection between variants present in a bacterial population is shown. Subsequent transcriptomic analysis is used to confirm the enormous influence of RNase $Y$ on $B$. subtilis physiology and to reveal possible causes for some specific rny related phenotypes.

In addition, a new experimental set up is established to assess the reasons for the loss of genetic competence not only in the rny mutant strain, but also in some other previously uncharacterized competence mutants of $B$. subtilis. This way, the reason for the loss of competence as well as other social behaviors of the mutant overproducing unknown $A B C$ transporter YtrBCDEF is described and further investigated. 


\section{Quasi-essentiality of RNase $Y$ in Bacillus subtilis is caused by its critical role in the control of mRNA homeostasis}

The results of this chapter are published in the following pre-print:

Benda, M., Woelfel, S., Gunka, K., Klumpp, S., Poehlein, A., Kálalová, D., Šanderová, H., Daniel, R., Krásný, L. and Stülke, J. (2020) Quasi-essentiality of RNase Y in Bacillus subtilis is caused by its critical role in the control of mRNA homeostasis. bioRxiv 2020.05.20.106237.

Author contribution:

$\mathrm{MB}, \mathrm{SW}$ and KG constructed the strains, evolved suppressors and assessed growth. SW performed CRISPR genome editing. MB purified the RNA polymerase, performed in vitro transcription assays and the evolution experiment. DK and HŠ constructed pBSURNAP. AP and RD sequenced the genomes. $\mathrm{MB}$ analyzed the sequences. $\mathrm{MB}, \mathrm{AP}$ and $\mathrm{RD}$ performed transcriptome analyses. SK build the RNA polymerase composition model. MB, KG, LK and JS designed the study. MB, LK and JS wrote the manuscript. 


\begin{abstract}
RNA turnover is essential in all domains of life. The endonuclease RNase $Y(r n y)$ is one of the key components involved in RNA metabolism of the model organism Bacillus subtilis. Essentiality of RNase $Y$ has been a matter of discussion, since deletion of the rny gene is possible, but leads to severe phenotypic effects. In this work, we demonstrate that the rny mutant strain rapidly evolves suppressor mutations to at least partially alleviate these defects. All suppressor mutants had acquired a duplication of an about $60 \mathrm{~kb}$ long genomic region encompassing genes for all three core subunits of the RNA polymerase $-\alpha, \beta, \beta^{\prime}$. When the duplication of the RNA polymerase genes was prevented by relocation of the $r p o A$ gene in the $B$. subtilis genome, all suppressor mutants carried distinct single point mutations in evolutionary conserved regions of genes coding either for the $\beta$ or $\beta^{\prime}$ subunits of the RNA polymerase that were not tolerated by wild type bacteria. In vitro transcription assays with the mutated polymerase variants showed a severe decrease in transcription efficiency. Altogether, our results suggest a tight cooperation between RNase $\mathrm{Y}$ and the RNA polymerase to establish an optimal RNA homeostasis in B. subtilis cells.
\end{abstract}

\title{
Introduction
}

Among all organisms, bacteria are the ones multiplying most rapidly. Under optimal conditions, the model bacteria Escherichia coli and Bacillus subtilis have generation times of 20 to 30 minutes. On the other hand, bacteria are exposed to a variety of changing environmental conditions, and due to their small size, the impact of environmental changes is particularly severe for bacterial cells. To adapt to these potentially rapidly changing conditions, bacteria have evolved a huge arsenal of systems to sense and respond to the environment. Especially in the competition between microorganisms, it is crucial that these responses are both rapid and productive. However, while regulatory events may be very rapid, there is an element of retardation in the system, and this is the stability of mRNA and protein molecules. If the continued activity of a protein may become harmful to the bacteria, it is important not only to prevent expression of the corresponding gene but also to take two important measures: (i) switch off the protein's activity and (ii) degrade the mRNA to exclude further production of the protein. The inactivation or even degradation of proteins is well documented in the model bacteria. For example, in both E. coli and B. subtilis the uptake of toxic ammonium is limited by a regulatory interaction of the ammonium transporter with GInK, a regulatory protein of the PII family (Coutts et al., 2002; Detsch and Stülke, 2003). Similarly, the uptake of potentially toxic potassium can be prevented by inhibition 
of potassium transporters at high environmental potassium concentrations, either by the second messenger cyclic di-AMP or by interaction with a dedicated modified signal transduction protein, PtsN (Lee et al., 2007; Corrigan et al., 2013; Gundlach et al., 2019). To prevent the accumulation of potentially harmful mRNAs, bacteria rely on a very fast mRNA turnover. Indeed, in E. coli and $B$. subtilis more than $80 \%$ of all transcripts have average half-lives of less than 8 minutes, as compared to about 30 minutes and 10 hours in yeast or human cells, respectively (Hambraeus et al., 2003; Yang et al., 2003; Bernstein et al., 2004; Geisberg et al., 2014). Thus, the mRNA turnover is much faster than the generation time. The high mRNA turnover rate in bacteria contributes to the fast adaptation even in rapidly growing cells. The rapid mRNA turnover is therefore a major factor to resolve the apparent growth speed-adaptation trade-off.

RNases are the key elements to achieve the rapid mRNA turnover in bacteria. Theses enzymes can degrade bulk mRNA in a rather unspecific manner, just depending on the accessibility of the RNA molecules as well as perform highly specific cleavages that serve to process an RNA molecule to its mature form. In all organisms, RNA degradation involves an interplay of endo- and exoribonucleases as well as other proteins such as RNA helicases that resolve secondary structures (Lehnik-Habrink et al., 2012; Durand et al., 2015; Redder, 2018; Tejada-Arranz et al., 2020). Often, these proteins form a complex called the RNA degradosome. In $E$. coli, the RNA degradosome is organized around the essential endoribonuclease RNase E (Carpousis, 2007; Mackie, 2013). RNase E consists of two parts, the N-terminal endoribonuclease domain that harbors the enzymatic activity and the C-terminal macromolecular interaction domain that serves as the scaffold for the degradosome components and is responsible for the binding of RNase E to the cell membrane (Khemici et al., 2008; Mackie, 2013). As mentioned above, RNase $E$ is essential for viability of the bacteria. An analysis of the contributions of the two parts of RNase $\mathrm{E}$ to its essentiality revealed that the enzymatically active $\mathrm{N}$-terminal domain is essential whereas the C-terminal interaction domain is dispensable (Kido et al., 1996). This suggests that the endoribonucleolytic attack on mRNA molecules is the essential function of RNase $E$, whereas the interaction with other degradosome components is not required for viability. This conclusion is supported by the fact, that the other components of the E. coli degradosome are also dispensable (Carpousis, 2007).

RNase $\mathrm{E}$ is widespread in proteobacteria, cyanobacteria, and actinobacteria, but absent from many firmicutes, ?-proteobacteria, or from bacteria of the Deinococcus-Thermus class. However, an efficient RNA-degrading machinery is important also for these bacteria to allow both rapid growth and adaptation. Indeed, these bacteria possess a different endoribonuclease, RNase Y (Commichau et al., 2009; Shahbabian et al., 2009). A depletion of RNase Y results in a two-fold increase of the average mRNA half-life in B. subtilis (Shahbabian et al., 2009). Similar to RNase E, 
RNase $\mathrm{Y}$ is a membrane protein, and it is capable of interacting with several proteins involved in RNA degradation. Among these proteins are the 5'-to-3' exoribonunclease RNase J1, polynucleotide phosphorylase, the RNA helicase CshA, the glycolytic proteins enolase and phosphofructokinase, and a protein complex composed of YaaT, YlbF, and YmcA (Commichau et al., 2009; Shahbabian et al., 2009; Lehnik-Habrink et al., 2011a; Newman et al., 2012; DeLoughery et al., 2016; Salvo et al., 2016). Many of these interactions are likely to be transient as judged from the distinct localization of RNase $Y$ and its interaction partners in the cell membrane and in the cytoplasm, respectively (Cascante-Estepa et al., 2016).

We are interested in the identification of the essential cellular components that are required for the viability of $B$. subtilis cells with the aim to construct strains that harbor only the minimal set of genes to fulfill the essential cellular functions (Commichau et al., 2013; Reuß et al., 2016; Reuß et al., 2017). For B. subtilis, RNase $Y$ and RNase J1 were originally described as being essential (Kobayashi et al., 2003; Hunt et al., 2006; Mathy et al., 2007; Commichau et al., 2009; Shahbabian et al., 2009). Interestingly, these two RNases are also present in the most genomereduced independently viable organism, Mycoplasma mycoides JCVI-syn3.0 (Hutchison et al., 2016). Both RNase J1 and RNase $Y$ are involved in the processing and degradation of a large number of RNA molecules in B. subtilis (Mäder et al., 2008; Lehnik-Habrink et al., 2011b)(Durand et al., 2012a; Laalami et al., 2013; DeLoughery et al., 2018). However, more recent studies demonstrated the possibility to delete the rnjA and rny genes, encoding the two RNases (Figaro et al., 2013; Šiková et al., 2020) and the dispensability of RNase $Y$ was confirmed in a global approach to inactivate all genes of $B$. subtilis (Koo et al., 2017).

Comprehensive knowledge on essential genes and functions is the key to construct viable minimal genomes. By definition, essential genes cannot be individually deleted in a wild type genetic background under standard growth conditions (Commichau et al., 2013). In this study, we have addressed the essentiality of RNase $Y$ in $B$. subtilis. While the rny gene could indeed be deleted, this was accompanied by the rapid acquisition of suppressor mutations that affect the transcription apparatus. We demonstrate that a strongly reduced transcription activity is required to allow stable growth of $B$. subtilis in the absence of RNase $Y$. Our results suggest that the accumulation of mRNA that cannot be degraded is the growth-limiting factor in strains lacking RNase $Y$. 


\section{Results}

\section{Inactivation of the rny gene leads to evolution of suppressor mutations affecting transcription}

RNase Y had been considered to be essential (Kobayashi et al., 2003; Commichau et al., 2009); however, two studies reported that the rny gene could be deleted from the genome (Figaro et al., 2013; Koo et al., 2017). The deletion leads to severe growth defects and morphological changes (Figaro et al., 2013). In an attempt to get a better understanding of the importance of RNase $Y$ for $B$. subtilis physiology, we deleted the rny gene in the genetic background of $B$. subtilis 168 . The colonies of the resulting strain, GP2501, were small and lysed rapidly. Moreover, the cells grew very slowly at low temperatures (below $22^{\circ} \mathrm{C}$ ). However, we observed the appearance of suppressor mutants after a few days. By analysis of such mutants we wished to gain a better understanding of the growth-limiting problem of the rny mutant. For this purpose, we isolated suppressor mutants in different experimental setups. First, the rny mutant GP2501 was adapted to growth in liquid LB medium at $22^{\circ} \mathrm{C}$ since the rny mutants had a severe growth defect at low temperatures. After the adaptation experiment, the culture was plated at $22^{\circ} \mathrm{C}$, and two colonies were isolated for further investigation. In addition to the adaptation experiment in liquid medium, we also evolved suppressors on solid LB agar plates both at $22^{\circ} \mathrm{C}$ and $37^{\circ} \mathrm{C}$. We isolated two mutants under each condition (see Fig. $6 \mathrm{~A}$ ).

Growth of the isolated strains was verified (see Fig. 6B, and Supplementary Figures S2 and S3), and for each selection scheme, one mutant was analysed by whole genome sequencing. In all cases, this confirmed the deletion of the rny gene and revealed the presence of an additional mutations. Strikingly, there was one feature common for all the suppressors tested, regardless of the isolation condition, which was not present in the progenitor strain GP2501: It was an identical genomic duplication of the approximately $60 \mathrm{~kb}$ long cts $R-p d a B$ region. This genomic segment is flanked by clusters of ribosomal RNA operons. Upstream of the duplicated region are the $r r n J$ and $r r n W$ operons, and downstream the $r r n l, r r n H$, and $r r n G$ operons (see Fig. 7A). This duplicated region contains 76 genes encoding proteins of various functions, among them proteolysis (ClpC), signal transduction (DisA), RNA modification (YacO, TruA), RNases (MrnC, Rae1), translation factors (EF-G, IF-1, EF-Tu), several ribosomal proteins, and proteins involved in transcription (NusG, RpoA, RpoB, RpoC, SigH). Strikingly, the genes for all three main subunits of the RNA polymerase $-r p o A, r p o B$ and $r p o C$ were present in the duplicated region. The observation, that this duplication was observed irrespective of the selective condition used to isolate suppressor 
mutants suggests that this duplication is relevant to overcome the poor growth associated with the loss of RNase Y. However, in addition, for each selection scheme we found additional mutations that affect genes involved in transcription.

A

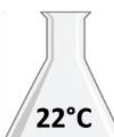

$22^{\circ} \mathrm{C}$

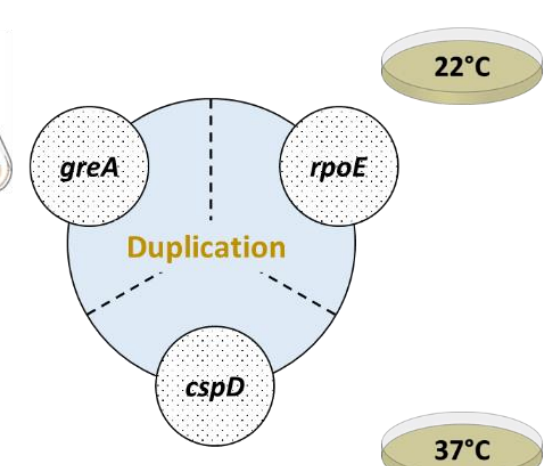

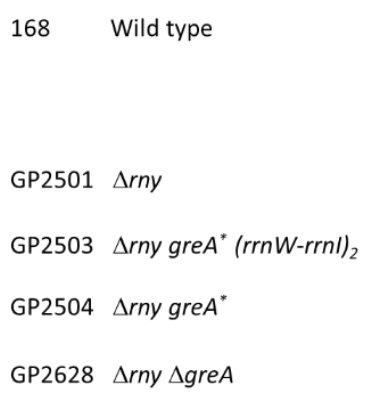

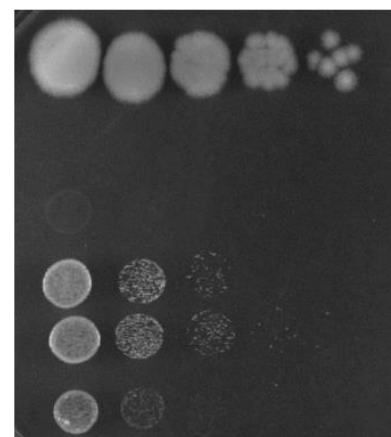

Figure 6: Suppressors of rny show increased growth at $22^{\circ} \mathrm{C}$

(A) Schematic depiction of different single nucleotide polymorphisms identified in the initial suppressor screen and their overlap with the duplication of $c t s R-p d a B$ region. (B) Serial drop dilutions comparing growth of the wild type strain 168, the rny mutant GP2501, its greA suppressors (GP2503, greA (Ser125Leu) $\left(\right.$ rrnW-rrnl) 2 ; GP2504, greA (Glu57Stop)) and the rny greA double mutant GP2628 on LB-agar plate at $22^{\circ} \mathrm{C}$. The picture was taken after 2 days of incubation.

For the selection in liquid medium at $22^{\circ} \mathrm{C}$, the suppressor mutant GP2503 had a point mutation that resulted in an amino acid substitution (S125L) in the greA gene encoding a transcription elongation factor (Kusuya et al., 2011). For the other suppressor mutant (GP2504) isolated under the same selective conditions, we sequenced the greA gene to test whether it had also acquired a mutation in this gene. Indeed, we found a different mutation in greA, resulting in the introduction of a premature stop codon after E56. Moreover, we evolved two additional suppressor mutants applying this adaptive scenario, and both contained frameshift mutations in greA that resulted in premature stop codons after amino acid 23 and 137 (GP2539 and GP2538, respectively; see Table S3).

The strain isolated on LB plates at $22^{\circ} \mathrm{C}$ (GP2637) had a deletion of the skin element, an amino acid substitution (Y55N) in the AdeR activator protein (Lin et al., 2012), and a short internal deletion in the rpoE gene encoding the $\delta$ subunit of RNA polymerase, which resulted in a frameshift after residue G66 (Juang and Helmann, 1994; Rabatinová et al., 2013). For the second mutant isolated at $22^{\circ} \mathrm{C}$ (GP3210), we re-sequenced the adeR and rpoE genes. While the adeR gene was identical to the wild type, we found an insertion of an adenine residue after position 87 of $r p o E$, resulting in a frameshift after 29 amino acids and premature stop codon after 38 amino acids. Therefore, the $r p o E$ but not the adeR mutation is likely to be required for the suppressor phenotype. 
The suppressor evolved at $37^{\circ} \mathrm{C}$ on LB plates (GP2636) contained a mutation resulting in the introduction of a premature stop at the eighth codon of the $\operatorname{cspD}$ gene encoding an RNA binding protein which has transcription antitermination activity in E. coli (Graumann et al., 1997; Bae et al., 2000). Sanger sequencing of the second suppressor isolated under the same condition (GP2678) also identified a mutation affecting $\operatorname{cspD}$, but this time in its ribosomal binding site (GGAGGA $\rightarrow$ GGAAGA).

Taken together, the duplication of the ctsR-pdaB genomic region was accompanied by specific additional suppressor mutation affecting transcription in every single suppressor mutant analysed. These mutations result in the inactivation of the greA gene in liquid medium at $22^{\circ} \mathrm{C}$, whereas the selective pressure on agar plates at $22^{\circ} \mathrm{C}$ and $37^{\circ} \mathrm{C}$ was directed at the inactivation of the RNA polymerase subunit RpoE or the RNA binding protein CspD, respectively (see Fig. 6A). It is therefore tempting to speculate that the inactivation of these genes combined with the ctsR-pdaB genomic duplication is causative for the suppression.

In order to test whether the inactivation of the $g r e A, r p o E$, or $\operatorname{cspD}$ genes alone is sufficient for the suppression of the rny mutant strain, we constructed the corresponding double mutants. As both rny and greA mutants are defective in genetic competence (Koo et al., 2017), the greA rny double mutant was obtained by transforming the wild type strain 168 with DNA molecules specifying both deletions simultaneously (see Table S3). For the greA and rpoE deletions, the double mutants did not phenocopy the original suppressor mutants, instead the gene deletions conferred only partial suppression (see Fig. 6B for the rny greA double mutant GP2628, and Supplementary Figure S2 for the rny rpoE double mutant GP3217). In the case of the rny $\operatorname{cspD}$ double mutant GP2615, complete suppression was observed (see Supplementary Figure S3). However, we cannot exclude that the mutant had already acquired the duplication of the $c t s R-p d a B$ genomic region. Thus, we conclude that the suppression depends on both, the duplication of the ctsR-pdaB region and the concomitant mutations that inactivate genes involved in transcription.

\section{Transcriptome analysis of the rny mutant and a suppressor strain}

As mentioned above, the deletion of greA allowed only partial suppression of the growth defect caused by the loss of RNase Y. However, the rny greA double mutant GP2628 eventually gave rise to a better suppressing mutant, GP2518. Whole genome sequencing of this strain revealed that in addition to the greA deletion it had only acquired the duplication of the ctsR$p d a B$ genomic region. Again, this highlights the relevance of the combination of the greA deletion and the $c t s R-p d a B$ duplication for suppression. 
To get insights into the global consequences of the suppressing mutations, we compared the transcriptomes of the wild type strain 168, the rny mutant GP2501, and the suppressor mutant GP2518 by RNA-Seq analysis. We identified 1,102 genes (corresponding to about $25 \%$ of all genes of B. subtilis) with at least two-fold differential expression in the $\Delta r n y$ strain GP2501 as compared to the wild type 168. It should be noted that the number of differentially expressed genes is likely to be underestimated, since about $50 \%$ of all genes are not or only very poorly expressed during vegetative growth (Rasmussen et al., 2009; Reuß et al., 2017). The rny gene is encoded within an operon with the $y m d B$ gene (Diethmaier et al., 2011); however, there was no polar effect on the expression of $y m d B$, suggesting that the observed changes are a direct result of the loss of RNase $Y$.

From the dataset mentioned above, 587 and 515 genes were down- and upregulated, respectively, in the rny strain. The most severe difference (more than 100-fold decrease) was observed for the $y x k C$ gene. This gene codes for protein of unknown function and is part of the $\sigma^{D}$ regulon (Serizawa et al., 2004). Interestingly, 14 out of the 30 most strongly downregulated genes are $\sigma^{\mathrm{D}}$ dependent (see Supplementary Table S1). This may be the result of the reduced expression of the $\operatorname{sig} D$ gene itself. Since $\sigma^{D}$ controls the expression of many genes responsible for motility as well as peptidoglycan autolysins (lytA, lytB,lytC, lytD and lytF) this reduced expression of target genes might cause the disordered cell wall of the rny deletion strain (Figaro et al., 2013). Among the most strongly upregulated genes (see Supplementary Table S1), many are members of the general stress response factor $\sigma^{B}$ regulon. Another set of upregulated genes is controlled by the sporulation specific sigma factors $\sigma^{\mathrm{F}}$ and $\sigma^{G}$, whose genes are also more than 4-fold upregulated. This is especially striking taking into an account that the rny mutant strain is not able to form spores (Figaro et al., 2013).

Importantly, we wanted to test whether the suppressor mutant had restored a wild typelike expression of genes that were affected by the loss of RNase Y. We found 461 genes with differential expression between the suppressor mutant GP2518 and the rny mutant GP2501. Of these, however, only some were returned towards the expression levels of the wild type (176 genes, see Supplementary Table S2), while for others, the mRNA levels were even more distant from the wild type. In total 115 genes upregulated in the rny strain showed reduced expression in the suppressor mutant. On the other hand, also 61 genes which were downregulated in the rny mutant, had increased their expression again in the suppressor mutant GP2518 (see Supplementary Table S2). Among these genes with restored expression, four (murAA, $\operatorname{tag} A, \operatorname{tag} B$, $y w p B)$ are essential, and only the expression of $y w p B$ encoding an enzyme of fatty acid biosynthesis is 2.4 -fold reduced in the rny mutant. This weak regulation suggests that fatty acid biosynthesis is not the growth-limiting factor for the rny mutant. In contrast, many of these genes 
with (partially) restored expression belong to prophage PBSX or are required for rather specific metabolic pathways. In conclusion, the evaluation of the genes which had their expression restored as a result of the suppressing mutations did not give a clear clue to the reason of suppression.

\section{Genomic separation of the genes encoding the core subunits of RNA polymerase}

As mentioned above, the region duplicated in all suppressor mutants contained genes encoding RNA modification enzymes, translation factors, ribosomal proteins, RNases, and proteins involved in transcription. MrnC and Rae1 are RNase Mini-III required for the maturation of $23 \mathrm{~S}$ rRNA and ribosome-associated A site endoribonuclease, respectively (Redko et al., 2008; Leroy et al., 2017). As our suppressor screen identified additional mutations related to transcription, we assumed that the translation-specific RNases encoded in this region might not be relevant for the suppression of the rny deletion. Therefore, we hypothesized that the duplication of the genes encoding the main three subunits of RNA polymerase made a major contribution to the selective advantage provided by the duplication.

A Chromosomal arrangement in wild type or GP2501 respectively

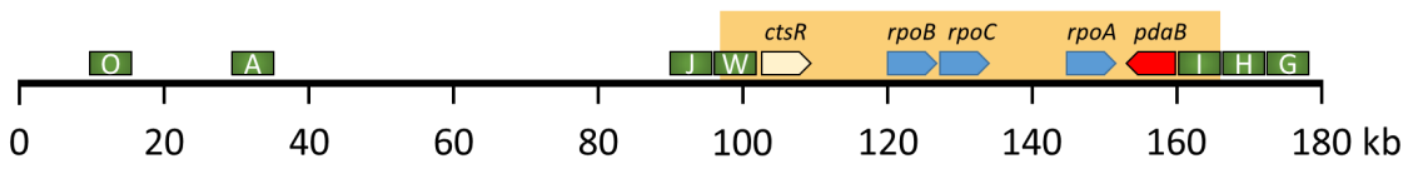

B

Chromosomal arrangement in newly created strain GP2903

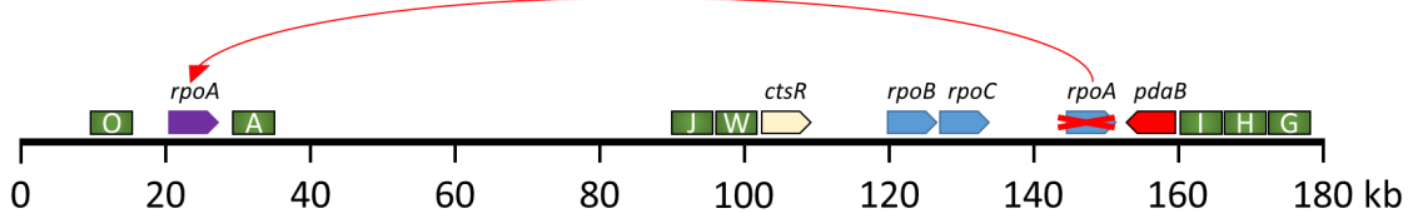

Figure 7: Genomic organization of the duplicated genomic region

(A) Schematic representation of the first $180 \mathrm{~kb}$ of the $B$. subtilis chromosome. The orange box indicates the duplicated region in the suppressors of rny strain GP2501. rRNA operons are depicted as green rectangles, RNA polymerase genes $r p o A, r p o B, r p o C$ as blue arrows, the $c t s R$ and $p d a B$ genes are shown in yellow and red, respectively. (B) Chromosomal relocation of the $r p o A$ gene. For the colour code, see above; the relocated $r p o A$ is shown as a purple arrow.

To test the idea that simultaneous duplication of all three genes for the RNA polymerase core subunits is the key for the suppression of the loss of RNase $Y$, we decided to interfere with this possibility. The duplicated region is located between two highly conserved rrn gene clusters which may facilitate the duplication event (see Fig. 7A). Therefore, we attempted to separate the core RNA polymerase genes by relocating the rpoA gene out of this genomic region flanked by the 
rrn operons. We assumed that if RNA polymerase was indeed the key to the original suppression, such a duplication would not be likely in the new background with relocated rpoA, since simultaneous duplication of all three RNA polymerase subunit genes would be disabled there. For this purpose, the rpoA gene kept under the control of its natural promoter $\mathrm{P}_{r p s}$ was placed between the $d g k$ and yaaH genes, and the original copy of rpoA was deleted (see Fig. 7B, Experimental procedures for details). We then compared the growth of the wild type strain 168 and the strain with the relocated rpoA GP2903 using a drop-dilution assay. No differences were observed, thus excluding a possible negative impact of the rpoA relocation on $B$. subtilis physiology (see Fig. S4).

Strain GP2903 was then used to delete the rny gene, and to isolate suppressor mutants. Indeed, even with the genomically separated RNA polymerase genes, suppressor mutations appeared upon the deletion of the rny gene encoding RNase $\mathrm{Y}$. There were three possibilities for the outcome of the experiment. First, the same genomic region as in the original suppressors might duplicate thus falsifying our hypothesis that the simultaneous duplication of all three genes encoding the core subunits of RNA polymerase is required for suppression. Second, both regions containing the $r p o A$ and $r p o B C$ genes might be duplicated. Third, in the new genetic background completely new suppressing mutations might evolve. Two of these suppressor mutants were subjected to whole genome sequencing. None of them had the duplication of the ctsR-pdaB region as in the original suppressors. Similarly, none of the mutants had the two regions containing the $r p o A$ and the $r p o B C$ genes duplicated. Instead, both mutants had point mutations in the RNA polymerase subunit genes that resulted in amino acid substitutions (GP2912: RpoC, R88H; GP2913: RpoB, G1054C; see Table S3). A mutation affecting RNA polymerase was also evolved in one strain (GP2915) not subjected to whole genome sequencing. In this case, the mutation resulted in an amino acid substitution (G45D) in RpoC.

An analysis of the localization of the amino acid substitutions in $\mathrm{RpoB}$ and RpoC revealed that they all affect highly conserved amino acid residues (see Fig. 8A). G1054 of RpoB and G45 of RpoC are universally conserved in RNA polymerases in all domains of life, and R88 of RpoC is conserved in the bacterial proteins. This high conservation underlines the importance of these residues for RNA polymerase function. The mutations G45D and R88H in RpoC affect the $\mathrm{N}$ terminal $\beta^{\prime}$ zipper and the zinc-finger like motif of the $\beta^{\prime}$ subunit, respectively, that are required for the processivity of the elongating RNA polymerase (Nudler et al., 1996; Nudler, 2009). G1054C in RpoB is located in the $\mathrm{C}$-terminal domain of the $\beta$ subunit that is involved in transcription termination (Clerget et al., 1995). In the three-dimensional structure of RNA polymerase, these regions of the $\beta$ and $\beta^{\prime}$ subunits are located in close vicinity opposite to each other in the region of 
the RNA exit channel which guides newly transcribed RNA out of the enzyme (see Fig. 8B; Nudler, 2009), and they are both in direct contact with DNA (Nudler et al., 1996).

The fact that several independent mutations affecting RNA polymerase were obtained in the suppressor screen strongly supports the idea that RNA polymerase is the key for the suppression. As the mutations affect highly conserved residues, they are likely to compromise the enzyme's activity. Based on the structural information, the mutations might weaken RNA polymerase-nucleic acid interactions and therefore, destabilize the transcription elongation complex which may result in increased premature termination and reduced RNA polymerase processivity. However, RNA polymerase is essential, therefore the mutations cannot inactivate the protein completely.

A
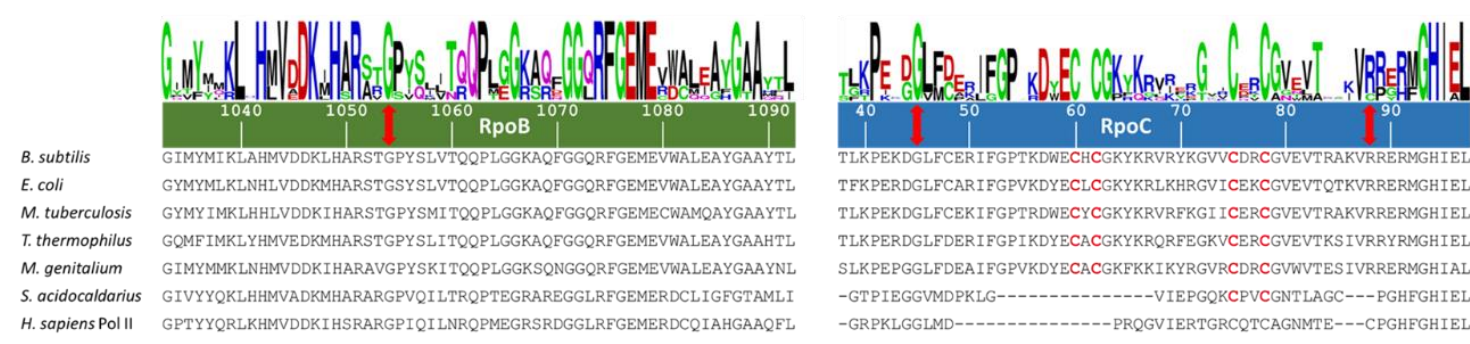
TFKPERDGLFCARIFGPVKDYECLCGKYKRLKHRGVICEKCGVEVTQTKVRRERMGHIEL TLKPEKDGLFCEKIFGPTRDWECYCGKYKRVRFKGI ICERCGVEVTRAKVRRERMGHIEL TLKPERDGLFDERIFGPIKDYECACGKYKRQRFEGKVCERCGVEVTKS IVRRYRMGHIEL SLKPEPGGLFDEAIFGPVKDYECACGKFKKIKYRGVRCDRCGVWVTESIVRRERMGHIAL -GTPIEGGVMDPKLG-------------VIEPGQKCPVCGNTLAGC---PGHFGHIEL -GRPKLGGLMD--------------PRQGVIERTGRCQTCAGNMTE---CPGHFGHIEL

B

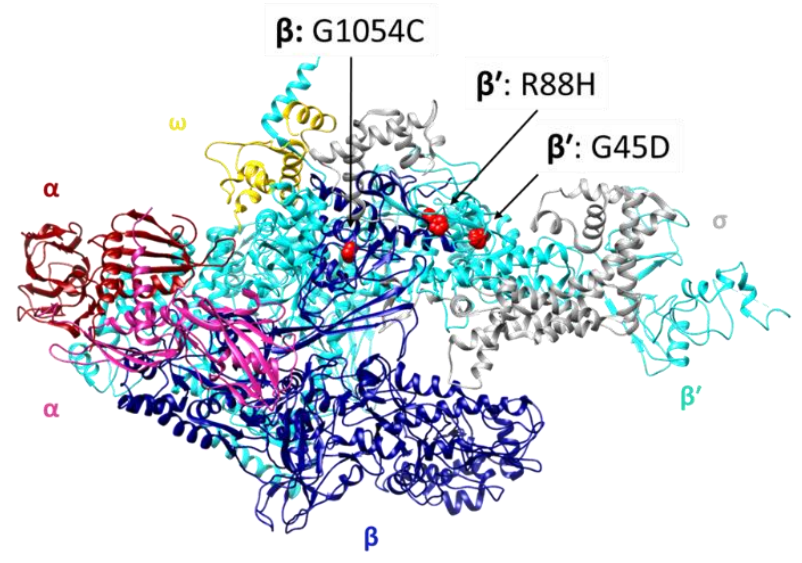

Figure 8: Suppressor mutations in RNA polymerase localize to evolutionary conserved regions

(A) Multiple sequence alignment of $\mathrm{RpoB}$ and $\mathrm{RpoC}$ sequences from various species, the numbering of amino acid residues is based on the $B$. subtilis sequence. The positions of mutations are indicated with red double head arrows, conserved cysteines involved in Zn-finger formation are shown in red. Logos were created as described (98). Abbreviations: B. subtilis, Bacillus subtilis; E. coli, Escherichia coli; M. tuberculosis, Mycobacterium tuberculosis; T. thermophilus, Thermus thermophilus; M. genitalium, Mycoplasma genitalium; S. acidocaldarius, Sulfolobus acidocaldarius; H. sapiens, Homo sapiens. (B) Localization of the mutations (indicated as red spheres) in the RNA polymerase shown at their corresponding position in the structure of $T$. thermophilus (PDB ID: 1IW7; 99). The two $\alpha$ subunits are shown in dark red and violet, respectively, the $ß$ subunit is shown in dark blue, $\beta^{\prime}$ in cyan, $\omega$ in gold and the $\sigma$ subunit is shown in grey. The image was created using UCSF Chimera (Pettersen et al., 2004). 


\section{Establishing the rpoB and rpoC mutations in wild type background}

Based on the essentiality of transcription, we expected that the mutations in $r p o B$ and rpoC that we have identified in the suppressor screen with the rny mutant and genomically separated RNA polymerase genes might adjust some of the properties of RNA polymerase. To study the consequences of these mutations for the RNA polymerase and hence also for the physiology of $B$. subtilis, we decided to introduce one of them (RpoC-R88H) into the wild type background of $B$. subtilis 168 . For this purpose, the CRISPR/Cas9 system designed for use in $B$. subtilis was employed (Altenbuchner, 2016). As a control, we used the same procedure to introduce a mutation in the rae1 gene, which is located nearby on the chromosome. Although this system readily allowed the introduction of a frameshift mutation (introduction of an extra T after $32 \mathrm{bp}$ ) in rae1 (strain GP2901), we failed to isolate genome-edited clones expressing the RpoC$\mathrm{R} 88 \mathrm{H}$ variant in multiple attempts. This failure to construct the RpoC-R88H variant in the wild type background suggests that the properties of the protein are altered in a way that is incompatible with the presence of an intact RNA degradation machine.

\section{Mutated RNA polymerases have highly decreased activity in vitro}

Since our attempts to study the effect of the mutations in vivo failed, we decided to test the properties of the mutant RNA polymerases using in vitro transcription. B. subtilis RNA polymerase is usually purified from a strain expressing His-tagged RpoC (Qi and Hulett, 1998). However, the loss of competence of the rny mutant and the lethality of the $r p o C$ mutation in the wild type background prevented the construction of a corresponding strain. To solve this problem, we used an approach to purify B. subtilis RNA polymerase from E. coli that had been successful before for RNA polymerase of Mycobacterium smegmatis (Kouba et al., 2019). Briefly, plasmid pBSURNAP containing genes rpoA, rpoB, rpoC, rpoE, rpoY, and rpoZ for the RNA polymerase subunits under control of an IPTG inducible promoter was constructed in a way that each individual gene for a subunit could be cleaved out using unique restriction sites and replaced with its mutant counterpart, yielding pGP2181 (RpoC-R88H) and pGP2182 (RpoB-G1054C) (for details of the construction, see Experimental procedures). The variant RNA polymerases were expressed in E. coli BL21 and purified via affinity chromatography and subsequent size exclusion chromatography.

We purified the wild type and two mutant RNA polymerases (RpoC-R88H and RpoBG1054C) and assessed their activity by in vitro transcription on three different templates, containing well-studied promoters of the veg and ilvB genes and the P1 promoter of the rrnB 
operon (Krásný and Gourse, 2004; Krásný et al., 2008). In agreement with previous results with wild type RNA polymerase (Sojka et al., 2011), this enzyme performed well on all three substrates. In contrast, the mutated variants of RNA polymerase exhibited a drastic decrease of transcription activity on all three promoters; for the RpoB-G1054C variant the transcripts were only barely detectable (Fig. 9A).

On many promoters, including the P1 promoter of the $\operatorname{rrnB}$ operon, B. subtilis RNA polymerase is sensitive to the concentration of the first transcribed nucleotide both in vitro and in vivo (Krásný and Gourse, 2004). This prompted us to compare the response of the wild type and the RpoC-R88H variant RNA polymerases to different concentrations of GTP, the initiation NTP for the $r r n B$ P1 transcript. As described before, transcription with the wild type enzyme increased gradually in response to the GTP concentration (Krásný and Gourse, 2004). In contrast, the mutated variant was saturated with a relatively low GTP concentration, suggesting that this important regulatory mechanism is not functional here (see Fig. 9B).
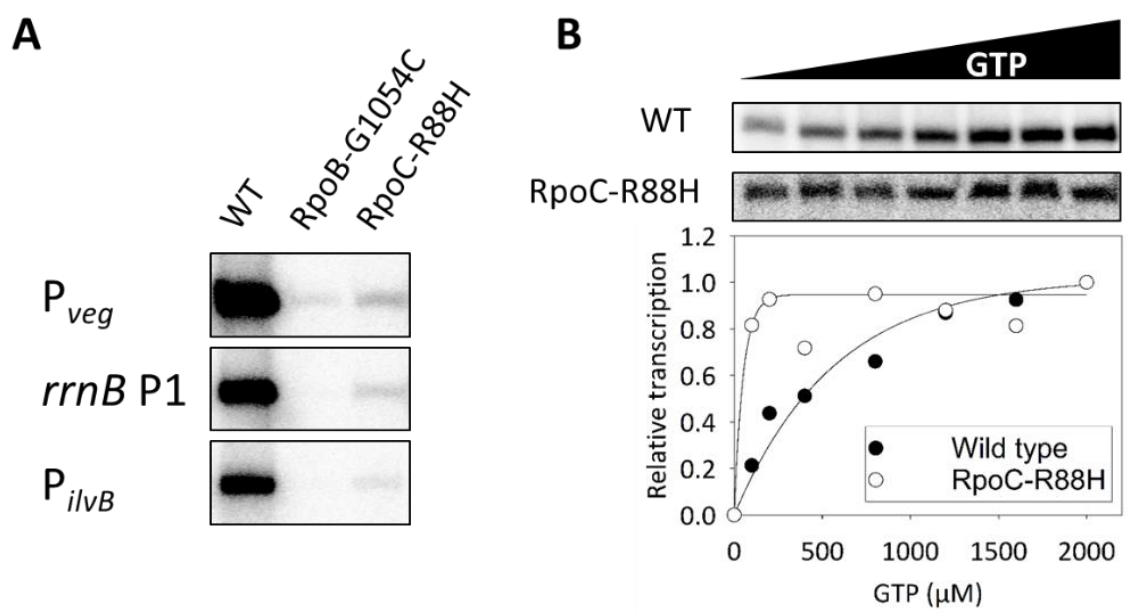

Figure 9: Comparison of transcriptional activity between RNA polymerase variants

(A) The RNA polymerase variants $(64 \mathrm{nM})$ were reconstituted with saturating concentrations of $\sigma^{A}(1: 10)$. Holoenzymes were used to initiate transcription on three promoters as indicated. A representative image from three independent experiments is shown. (B) Transcription from the $r$ rnB P1 promoter in dependence on increasing concentration of iNTP (GTP). The intensity of the transcripts generated by RNA polymerase containing RpoC-R88H was adjusted for better visibility. The relative activity of this mutant RNA polymerase was $2.5 \%$ of the wild type RNA polymerase at $2,000 \mu \mathrm{M}$ GTP. The graph shows average of two replicates normalized for maximal transcription of each polymerase (set as 1 ).

Taken together, our results suggest that a reprogramming of the properties of RNA polymerase as indicated by a substantial reduction in RNA polymerase activity and its altered ability to be regulated by iNTPs allows the suppressor mutants to overcome the loss of RNase $\mathrm{Y}$. 


\section{A pre-existing duplication of the genomic region containing rpoA and $r p o B C$ is fixed in response to the deletion of $r n y$}

The screen for suppressor mutations that facilitate growth of strains lacking RNase $Y$ yielded two classes of mutants: the first set harboured mutations in genes involved in transcription ( $g r e A, r p o E$, or $\operatorname{cspD}$ ) in addition to a duplication of the chromosomal region encoding the core subunits of RNA polymerase. The second class had point mutations affecting the $\beta$ or $\beta^{\prime}$ subunits of RNA polymerase that result in strongly decreased transcription activity. At a first glance, these results seem to be conflicting. Considering RNA degradation as the function of RNase $Y$, it seemed plausible that the selective pressure caused by deletion of $r n y$ should result in alleviating the stress from mRNA accumulation. This seems to be the case in the second class of suppressors (see above), whereas the logic behind the duplication seems to be less obvious. Importantly, this duplication was always accompanied by one of the other aforementioned mutations affecting transcription. In an attempt to determine the order of the evolutionary events in these suppressors we established a method to detect the presence of the duplication without whole genome sequencing. For this, we made use of a pair of oligonucleotides that binds to the $p d a B$ and $c t s R$ genes giving a product of about $10 \mathrm{~kb}$, if the region is duplicated or amplified but no product in the absence of duplication or amplification (see Fig. 10A). This PCR product was very prominent for the strain GP2636 that is known to carry the duplication. However a band was also observed in the wild type strain 168, indicating that the duplication is present in a part of the population independent from the selective pressure exerted by the rny deletion (Fig. 10B).

It is well-established that genomic duplications or amplifications occur frequently in bacterial populations, even in the absence of selective pressure (Andersson and Hughes, 2009). In Salmonella typhimurium, rrn operons have been shown to be a hotspot of gene duplications or amplifications (Anderson and Roth, 1981). Since evolution of such a genomic duplication is dependent on homologous recombination, we performed the PCR also on the recA mutant GP2542, which is defective in homologous recombination and thus unable to amplify chromosomal regions (Dormeyer et al., 2017; Reuß et al., 2019). Indeed, in this case we did not obtain even a faint band. Interestingly, the genomic duplication can also be observed in cells having the core subunits of RNA polymerase at distinct genomic regions (GP2903). For the derived suppressor mutant GP2912 that carries a point mutation in rpoC, the band indicating the presence of the duplication was also detectable by PCR analysis although the duplication could not be detected by genome sequencing. This apparent discrepancy is most easily resolved by assuming that the duplication was present only in a small subpopulation (as observed for the wild type strain) and therefore only detectable by the very sensitive PCR assay. 
$\underline{\text { WT }}$

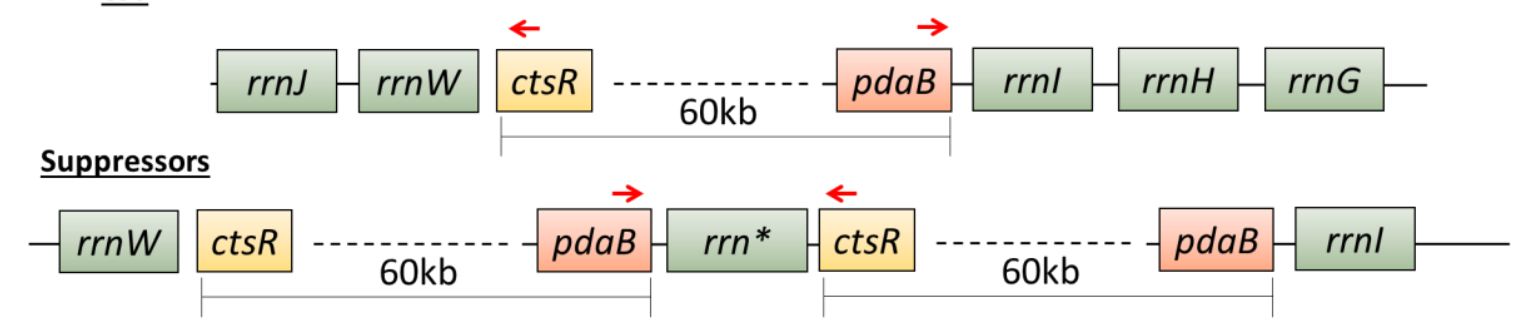

B

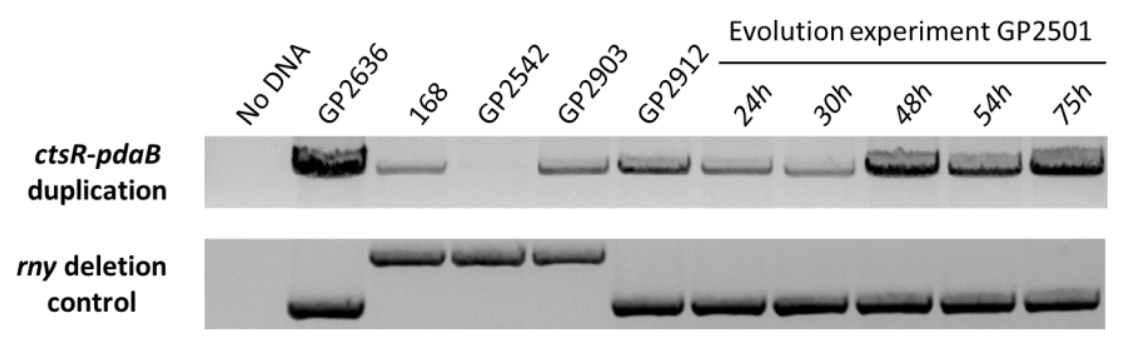

Figure 10: Duplication of the ctsR-pdaB region in suppressors of the rny mutant GP2501

(A) Schematic representation of the ctsR-pdaB region and its duplication in suppressors of GP2501. In the suppressors, a chimeric $r r n$ operon (shown as $r r n^{*}$ ) is located between the $p d a B$ and ctsR genes. The binding sites of the oligonucleotides used for the PCR detection of the duplication is indicated by red arrows. (B) Upper panel: The PCR product obtained by PCR using primers binding to $p d a B$ and ctsR genes indicating presence of the duplication. Lower panel: The PCR product for the amplification of the rny region. Note the $5 \mu \mathrm{l}$ of the PCR product were loaded in the upper panel, and $1 \mu \mathrm{l}$ in the lower panel

Obviously, the different genomic and genetic backgrounds of the rny mutants generate distinct selective forces: While the duplication is not fixed in strains with separated rpo genes, it seems to become fixed in the suppressor mutants that have the rpo genes in one genomic region. To investigate the order of evolutionary events, we cultivated the rny mutant strain GP2501 for 75 hours and monitored the status of the rpoA-rpoBC chromosomal region by PCR (see Fig. 10B). The initial sample for the rny mutant GP2501 that was used for the experiment, already revealed the presence of the duplication in a small sub-population similar to the wild type strain. This supports the finding that the duplication is present irrespective of any selection. The band corresponding to the duplicated pdaB-ctsR region became more and more prominent in the course of the experiment, after 75 hours it was comparable to the signal obtained with strain GP2636 that carries the duplication. As a control, we also amplified the genomic region of the rny gene. In the wild type strain, this PCR product has a size of $2.5 \mathrm{~kb}$, whereas the replacement of rny by a spectinomycin resistance gene resulted in a product of $2 \mathrm{~kb}$. Importantly, the intensity of this PCR product did not change during the course of the evolution experiment, thus confirming that the increased intensity of the product for the pdaB-ctsR region represents the spread of the duplication in the bacterial population. To verify the duplication and to check for the presence of accompanying mutations, we subjected genomic DNA of the strain obtained in this evolution experiment after 75 hours (GP3211) to whole genome sequencing. The sequencing confirmed 
presence of the duplication, but did not reveal any additional suppressor mutation. Based on this result, we can assume that upon deletion of rny the bacteria first fixed the duplication of the $p d a B$-ctsR region and then, later, may acquire the point mutations affecting greA, $r p o E$, or $\operatorname{csp} D$.

\section{Perturbing stoichiometry of transcription complexes reduces RNA polymerase activity}

In the investigation of suppressor mutants we have found suppressor mutants that exhibited severely reduced RNA polymerase activity as well as suppressor mutants with increased copy number of core RNA polymerase subunit genes. In the latter mutants, one might expect that the increased copy number of RNA polymerase core subunit genes would result even in increased transcription, apparently in contradiction to the other set of suppressors. However, the outcome of gene duplication may just be the opposite: The RNA polymerase is a complex multi-protein machine that contains several important proteins in addition to the core subunits. As these factors, including the sigma factor and other subunits like RpoE, RpoY and RpoZ (Juang and Helmann, 1994; Doherty et al., 2010; Delumeau et al., 2011; Rabatinová et al., 2013; Keller et al., 2014) as well as transcription factors like GreA and NusA (Davies et al., 2005; Kusuya et al., 2011) bind to the RNA polymerase via the core subunits, the perturbation of the normal evolved equilibrium between the RNA polymerase core subunits and transcription factors is likely to result in the formation of abortive incomplete complexes that are not fully active in transcription. To obtain a quantitative estimate for the formation of incomplete complexes, we turned to modelling.

We estimated the stoichiometry of the complexes in the wild type from proteomic mass fractions of the components (Reuß et al., 2017), calculating the number ratio of the subunit or transcription factor to the core RNA polymerase. These data indicate that GreA and the RpoZ subunit are in excess of core RNA polymerase, but not NusA, $\sigma^{A}$ as well as the RpoE and RpoY subunits (Fig. 11A). Since $\sigma^{A}$ is needed during initiation of transcription and NusA during elongation, we make the simplifying assumption that these two factors bind to the core RNA polymerase subsequently with NusA replacing $\sigma^{A}$ during transcription elongation, such that only one of them is present in the complex and their numbers can effectively be summed up ( $O^{\prime}$ Reilly et al., 2020). Taken together, their number is only slightly smaller than that of core RNA polymerases $(90 \%)$. This means that, in the wild type, $90 \%$ of all core RNA polymerases can form a complete complex including GreA, RpoZ and either $\sigma^{A}$ or NusA depending on the stage of transcription.

This fraction is strongly reduced if the core subunits are duplicated relative to the other subunits: To see that we make the assumption that the small subunits and transcription factors 
bind to the core RNA polymerase independently of each other. Upon duplication, the core RNA polymerase is in excess of all subunits and factors and thus a variety of partial complexes can be formed. The probability that a complex contains a specific set of factors is obtained by interpreting the stoichiometric ratio of a subunit to core as the probability that a core RNA polymerase will bind the subunit. The combinatorics of those probabilities give the fractions of the various complexes. For a complete complex consisting of core RNA polymerase, GreA, RpoZ and $\sigma^{A} /$ NusA, this leads to $0.6 \times 0.85 \times(0.15+0.3) \approx 0.23$, indicating that a duplication of the core subunits may result in a reduction of the fraction of complete complexes down to $23 \%$ of the core RNA polymerases in contrast to $90 \%$ in the wild type strain. This will result in reduced transcription activity even if there are twice as many core RNA polymerases than in the wild type since a variety of incomplete complexes containing different subsets of the subunits and transcription factors are formed (Fig. 11B). In the same way, we can estimate the fraction of complexes that contain the RpoE and RpoY subunits in addition. These complexes make up only $59 \%$ of all core RNA polymerases already in the wild type and their fraction is reduced down to $8 \%$ upon core duplication. Thus, a duplication of the core subunit genes is indeed expected to result in a strong decrease of the transcription activity.

A

\begin{tabular}{ll|l|l|l|l|l|l} 
Rpoz RpoE, RpoY & \multicolumn{6}{l}{ Relative abundance in WT } \\
\hline core & $\begin{array}{l}\text { core } \\
\left(\alpha_{2} \beta \beta^{\prime}\right)\end{array}$ & $\sigma^{A}$ & NusA & GreA & Rpoz & RpoE & RpoY \\
\cline { 2 - 8 } & 1 & 0.3 & 0.6 & 1.2 & 1.7 & 0.3 & 0.5
\end{tabular}

B

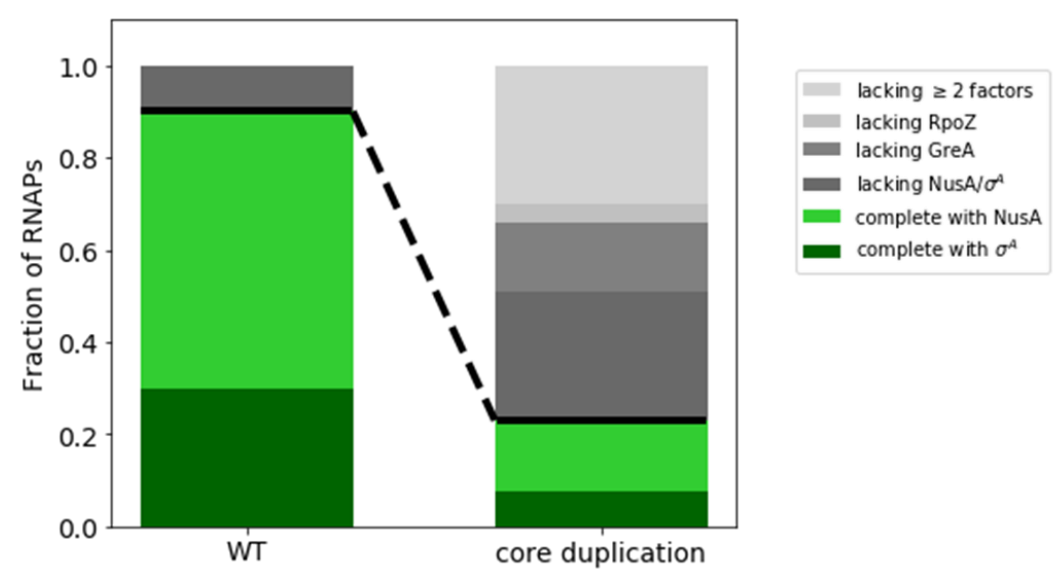

Figure 11: The duplication of the genes for core RNA polymerase is likely to result in the formation of incomplete RNA polymerase complexes

(A) Relative abundance/stoichiometry of RNA polymerase subunits and associated factors from proteomics data (Reuß et al., 2017). (B) Fractions of core RNA polymerase in different complete (green) and incomplete (grey) complexes estimated based on the relative abundance in (A) for the wild type and for the core duplication strain, where the relative abundance of core subunits is doubled compared to all other subunits. 


\section{Discussion}

RNases $\mathrm{E}$ and $\mathrm{Y}$ are the main players in RNA degradation in E. coli and B. subtilis, respectively. Recently, it has been estimated that about $86 \%$ of all bacteria contain either RNase $\mathrm{E}$ or RNase Y (or, sometimes, both) supporting the broad relevance of these two enzymes (TejadaArranz et al., 2020). While RNase E of E. coli is essential (Hammarlöf et al., 2015), conflicting results concerning the essentiality of RNase $\mathrm{Y}$ have been published (Kobayashi et al., 2003; Hunt et al., 2006; Commichau et al., 2009; Figaro et al., 2013; Koo et al., 2017). In this study, we have examined the properties of $B$. subtilis mutants lacking RNase $Y$ due to deletion of the corresponding rny gene. We observed that the rny mutant grew poorly, and rapidly acquired secondary mutations that suppressed, at least partially, the growth defect caused by the deletion of the rny gene. Thus, we conclude that RNase $Y$ is in fact quasi-essential (Hutchison et al., 2016) for $B$. subtilis, since the mutant cannot be stably propagated on complex medium without acquiring suppressor mutations.

A lot of effort has been devoted to the understanding of the reason(s) of the (quasi)essentiality of RNases $E$ and $Y$ for $E$. coli and B. subtilis, respectively. Initially, it was assumed that the essentiality is caused by the involvement of these RNases in one or more key essential processing event(s) that may affect the mRNAs of essential genes as has been found for $B$. subtilis RNase III and E. coli RNase P (Lehnik-Habrink et al., 2011b; Durand et al., 2012a; Durand et al., 2012b; Laalami et al., 2013; Mohanty et al., 2020). However, such a target was never identified. Instead, different conclusions were drawn from suppressor studies with $E$. coli rne mutants lacking RNase E: some studies reported suppression by the inactivation or overexpression of distinct genes, such as deaD encoding a DEAD-box RNA helicase and ppsA encoding phosphoenolpyruvate synthetase, respectively (Tamura et al., 2012; Tamura et al., 2016). In addition, the processing and degradation of the essential stable RNAs, such as tRNAs and rRNAs was shown to be an essential function of RNase E (Sulthana et al., 2016). Yet another study suggested that mRNA turnover is the growth-limiting factor of the E. coli rne mutant (Hammarlöf et al., 2015). The results presented here lend strong support to the idea that the main task of RNase $\mathrm{Y}$ in $B$. subtilis is the control of intracellular mRNA concentration via the initiation of mRNA degradation. The transcriptome analysis with the rny mutant and a suppressor mutant revealed that only a limited number of genes shows restored expression in the suppressor mutant. Moreover, most of these genes are part of the prophage PBSX or encode very specific metabolic functions. In addition, irrespective of the conditions used in the different suppressor screens, we identified a coherent set of mutations that resulted in improved growth of the B. subtilis rny mutant. The initial mutants carry a duplication of the chromosomal region that contains the genes 
for the core subunits of RNA polymerase (RpoA, RpoB, RpoC) and point mutations in greA, rpoE, and $\operatorname{csp} D$ that all affect transcription. If this duplication was prevented by genomically separating the RNA polymerase genes, we found suppressor mutants affecting the core subunits of RNA polymerase which result in strongly compromised transcription activity. Taken together, these findings suggest that the (quasi)-essentiality of RNases $E$ and $Y$ is related to their general function in initiating mRNA turnover rather than to the processing of specific RNA species. This idea is further supported by two lines of evidence: First, mutations that mimic a stringent response and therefore reduce RNA polymerase activity suppressed the growth defect of an rne mutant, and second, artificial expression of RNase $\mathrm{Y}$ or of the ribonucleases RNase $\mathrm{J} 1$ or $\mathrm{J} 2$ from B. subtilis partially suppressed the $E$. coli strain lacking RNase $E$, but only under specific growth conditions (Tamura et al., 2017; Himabindu and Anupama, 2017).

With the initiation of global mRNA degradation as the (quasi)-essential function of RNases $E$ and $Y$ in $E$. coli and B. subtilis, respectively, one might expect that the overexpression of other RNases might compensate for their loss. By analogy, such a compensation has been observed for the essential DNA topoisomerase I of $B$. subtilis, which could be replaced by overexpression of topoisomerase IV (Reuß et al., 2019). However, in all the seven suppressor mutants analyzed by whole genome sequencing (see Table S3), we never observed a mutation affecting any of the known RNases of $B$. subtilis. Similarly, no such compensatory mutations resulting from overexpression of other cognate RNases have been found in suppressor screens for $E$. coli RNase E. While RNase $\mathrm{Y}$ does not have a paralog in B. subtilis, E. coli possesses the two related RNases $\mathrm{E}$ and G. However, not even the overexpression of RNase $\mathrm{G}$ allowed growth of an E. coli rne mutant (Deana and Belasco, 2004; Chung et al., 2010) suggesting that RNase $G$ has a much more narrow function than RNase $E$ and that none of the other RNases in either bacterium is capable of initiating global mRNA degradation. Interestingly, as mentioned above, RNase J1 could partially replace RNase E in E. coli (Tamura et al., 2017), whereas it is not able to replace RNase $Y$ in $B$. subtilis. This difference could be due to the fact that RNase J1 provides an additional pathway to initiate mRNA degradation in B. subtilis, which is not naturally present in E. coli. This idea is further supported by the observation that a $B$. subtilis strain lacking both RNases $Y$ and $J 1$ could never be constructed (Figaro et al., 2013).

An interesting result of this study was the apparent contradiction between the isolation of suppressor mutants with increased copy number of core RNA polymerase subunit genes in one setup, intuitively suggesting increased transcription activity, and the isolation of mutants that exhibited severely reduced RNA polymerase activity in the other setup. We therefore tested with a theoretical model whether duplication of the core subunits leads to abortive incomplete complexes, as the composition of the RNA polymerase complex might be perturbed by the 
duplication of the core. The calculations indicate that most (90\%) of the core RNA polymerases in the wild type are associated with GreA and RpoZ as well as either sigma or NusA, depending on their stage in the transcription process, while upon core subunit gene duplication, the fraction of complete complexes, i.e. complexes associated to all these factors, is strongly reduced (to $23 \%$ ). Thus, the model shows that perturbing the stoichiometry of the transcription machinery results in a strong reduction of the fraction of core RNA polymerases that assemble a complete complex. As a consequence, a duplication of the core subunit genes is indeed expected to result in a strong decrease of the transcription activity, resolving the apparent contradiction.

In each organism, an optimal trade-off between RNA synthesis and degradation must be adjusted to allow optimal growth. Obviously, the loss of the major RNA decay-initiating enzyme will bring this adjustment out of equilibrium. This idea is supported by the observation that reduced RNA degradation in $B$. subtilis is accompanied by the acquisition of mutations that strongly reduce transcription activity of the RNA polymerase. Actually, the reduction of activity was so strong that it was not tolerated in a wild type strain with normal RNA degradation. This indicates that the suppressor mutants have reached a new stable equilibrium between RNA synthesis and degradation, which, however, is not optimal as judged from the reduced growth rates of the suppressor mutants as compared to the wild type strain. It has already been noticed that generation times and RNA stability are directly related (Yang et al., 2003; Rustad et al., 2013). This implies that a stable genetic system requires a balance between transcription and RNA degradation to achieve a specific growth rate. In bacteria, rapid growth requires high transcription rates accompanied by rapid RNA degradation. The association between RNA polymerase and components of the RNA degrading machinery, as shown for B. subtilis and Mycobacterium tuberculosis might be a factor to achieve this coupling between RNA synthesis and degradation (Delumeau et al., 2011; Płociński et al., 2019).

In conclusion, our study suggests that the initiation of mRNA degradation to keep the equilibrium between RNA synthesis and degradation is the function of RNase $Y$ that makes it quasi-essential for B. subtilis. In addition to RNase $Y$, RNase $J 1$ is also quasi-essential for this bacterium. In the future, it will be interesting to understand the reasons behind the critical role of this enzyme as well in order to get a more comprehensive picture of the physiology of RNA metabolism. 


\section{Experimental procedures}

\section{Bacterial strains, plasmids and growth conditions}

All B. subtilis strains used in this study are listed in Table S3. All strains are derived from the laboratory strain 168 (trpC2). B. subtilis and E. coli cells were grown in Lysogeny Broth (LB medium; Sambrook et al., 1989). LB plates were prepared by addition of $17 \mathrm{~g}$ Bacto agar/I (Difco) to LB (Sambrook et al., 1989). The plasmids are listed in Table S4. Oligonucleotides are listed in Table S5.

\section{DNA manipulation and genome sequencing}

B. subtilis was transformed with plasmids, genomic DNA or PCR products according to the two-step protocol (Sambrook et al., 1989; Kunst and Rapoport, 1995). Transformants were selected on LB plates containing erythromycin $(2 \mu \mathrm{g} / \mathrm{ml})$ plus lincomycin $(25 \mu \mathrm{g} / \mathrm{ml})$, chloramphenicol $(5 \mu \mathrm{g} / \mathrm{ml})$, kanamycin $(10 \mu \mathrm{g} / \mathrm{ml})$, or spectinomycin $(250 \mu \mathrm{g} / \mathrm{ml})$. Competent cells of E. coli were prepared and transformed following the standard procedure (Sambrook et al., 1989) and selected on LB plates containing kanamycin $(50 \mu \mathrm{g} / \mathrm{ml})$. S7 Fusion DNA polymerase (Mobidiag, Espoo, Finland) was used as recommended by the manufacturer. DNA fragments were purified using the QIAquick PCR Purification Kit (Qiagen, Hilden, Germany). DNA sequences were determined by the dideoxy chain termination method (Sambrook et al., 1989). Chromosomal DNA from B. subtilis was isolated using the peqGOLD Bacterial DNA Kit (Peqlab, Erlangen, Germany). To identify the mutations in the suppressor mutant strains GP2503, GP2518, GP2636, GP2637, GP2912, GP2913, and GP3211 (see Table S3), the genomic DNA was subjected to whole-genome sequencing. Concentration and purity of the isolated DNA was first checked with a Nanodrop ND1000 (PeqLab, Erlangen, Germany) and the precise concentration was determined using the Qubit $^{\circledR}$ dsDNA HS Assay Kit as recommended by the manufacturer (Life Technologies GmbH, Darmstadt, Germany). Illumina shotgun libraries were prepared using the Nextera XT DNA Sample Preparation Kit and subsequently sequenced on a MiSeq system with the reagent kit v3 with 600 cycles (Illumina, San Diego, CA, USA) as recommended by the manufacturer. The reads were mapped on the reference genome of B. subtilis 168 (GenBank accession number: NC_000964) (Barbe et al., 2009). Mapping of the reads was performed using the Geneious software package (Biomatters Ltd., New Zealand) (Kearse et al., 2012). Frequently occurring hitchhiker mutations (Reuß et al., 2019) and silent mutations were omitted from the screen. The resulting genome sequences were compared to that of our in-house wild type strain. Single nucleotide 
polymorphisms were considered as significant when the total coverage depth exceeded 25 reads with a variant frequency of $\geq 90 \%$. All identified mutations were verified by PCR amplification and Sanger sequencing. Copy numbers of amplified genomic regions were determined by dividing the mean coverage of the amplified regions by the mean coverage of the remaining genome as described previously (Dormeyer et al., 2017; Reuß et al., 2019).

\section{Construction of deletion mutants}

Deletion of the $r n y, r p o A$, and $\operatorname{cspD}$ genes was achieved by transformation with PCR products constructed using oligonucleotides to amplify DNA fragments flanking the target genes and intervening antibiotic resistance cassettes as described previously (Youngman, 1990; Guérout-Fleury et al., 1995; Wach, 1996). The identity of the modified genomic regions was verified by DNA sequencing.

\section{Chromosomal relocation of the rpoA gene}

To construct a strain in which the genes for the core subunits of RNA polymerase are genomically separated, we decided to place the rpoA gene between the $\mathrm{dgk}$ and yaaH genes, and then to delete the original copy of the gene. First, the rpoA gene was fused in a PCR reaction with its cognate promoter and a chloramphenicol resistance gene at the $5^{\prime}$ and $3^{\prime}$ ends, respectively. In addition, the amplified $d g k$ and yaaH genes were fused to this construct to direct the integration of the construct to the dgk-yaaH locus. The fusion of PCR products was achieved by overlapping primers. The final product was then used to transform B. subtilis 168. Correct insertion was verified by PCR amplification and sequencing. The resulting strain was B. subtilis GP2902. In the second step, the original rpoA gene was replaced by a kanamycin resistance gene as described above, leading to strain GP2903.

\section{Genome editing}

Introduction of genetic changes in genes for RNA polymerase subunit RpoC or the nonessential RNase Rae1 at their native locus was attempted using CRISPR editing as described (Altenbuchner, 2016). Briefly, oligonucleotides encoding a 20 nucleotide gRNA with flanking Bsal sites and a repair fragment carrying mutations of interest with flanking Sfil restriction sites were cloned sequentially into vector pJOE8999 (Altenbuchner, 2016). The resulting plasmids pGP2825 and pGP2826 were used to transform recipient $B$. subtilis strain 168 and cells were plated on 10 $\mu \mathrm{g} / \mathrm{ml}$ kanamycin plates with $0.2 \%$ mannose. Transformation was carried out at $30^{\circ} \mathrm{C}$ since 
replication of pJOE8999 derivatives is temperature-sensitive. The transformants were patched on LB agar plates and incubated at the non-permissive temperature of $50^{\circ} \mathrm{C}$. The loss of the vector was verified by the inability of the bacteria to grow on kanamycin plates. The presence of the desired mutation in rae1 or $r p o C$ was checked via Sanger sequencing. While the desired mutation could be introduced into the rae1 gene, this was not the case for rpoC.

\section{Construction of the expression vector pBSURNAP}

To facilitate the purification of different variants of $B$. subtilis RNA polymerase, we expressed and purified the core subunits of the RNA polymerase and the sigma factor separately in E. coli. For the expression of the core subunits, we cloned the corresponding B. subtilis genes into the backbone of a pET28a derivative as follows. The pRMS4 vector (a pET28a derivative, Kouba et al., 2019) containing Mycobacterium smegmatis RNA polymerase core subunit genes was used as a template to create an analogous vector containing the genes $r p o A, r p o Z, r p o E, r p o Y$, and $r p o B C$. The construct was designed to allow removal/substitution of each gene via unique restriction sites (see Fig. S1). DNA encoding rpoA, rpoZ, rpoE and rpoY genes was cloned as one single fragment (purchased as Gene Art Strings from Invitrogen) via Xbal and Notl restriction sites. The $r p o B$ and $r p o C$ genes were amplified by PCR using genomic DNA of $B$. subtilis 168 as a template and inserted into the plasmid via Notl and Ncol or Ncol and Kpnl restriction sites, respectively. The $r p o C$ gene was inserted with a sequence encoding a $8 x H i s$ tag on the $3^{\prime}$ end. The cloned construct was verified by DNA sequencing. The final vector, pBSURNAP, encodes a polycistronic transcript for expression of all six RNA polymerase core subunits. Expression is driven from an IPTG-inducible T7 RNAP-dependent promoter. Each gene is preceded by a ShineDalgarno sequence (AGGAG) except for rpoC. RpoB-RpoC are expressed as one fused protein connected by a short linker ( 9 amino acid residues) to decrease the possibility that $E$. coli subunits would mix with B. subtilis subunits as done previously for RNA polymerase from Mycobacterium bovis (Czyz et al., 2014). The full sequence of pBSURNAP has been deposited in GenBank under Accession No. MT459825. The mutant alleles of $r p o B$ and $r p o C$ were amplified from the mutant strains GP2913 and GP2912 and introduced into pBSURNAP by replacing the wild type alleles as $\mathrm{Notl} / \mathrm{Ncol}$ and $\mathrm{Ncol} / \mathrm{Kpnl}$ fragments, respectively. The resulting plasmids were pGP2181 (RpoC$\mathrm{R} 88 \mathrm{H})$ and pGP2182 (RpoB-G1054C). 


\section{Purification of $B$. subtilis RNA polymerase from $E$. coli cells}

For purification, E. coli BL21 carrying pBSURNAP or the plasmids specifying the mutant alleles was cultivated in LB medium containing kanamycin $(50 \mu \mathrm{g} / \mathrm{ml})$. Expression was induced by the addition of IPTG (final concentration $0.3 \mathrm{mM}$ ) to logarithmically growing cultures $\left(\mathrm{OD}_{600}\right.$ between 0.6 and 0.8 ), and cultivation was continued for three hours. Cells were harvested and the pellets from $1 \mathrm{I}$ of culture medium were washed in $50 \mathrm{ml}$ buffer $\mathrm{P}(300 \mathrm{mM} \mathrm{NaCl}, 50 \mathrm{mM}$ $\mathrm{Na}_{2} \mathrm{HPO}_{4}, 3 \mathrm{mM} \beta$-mercaptoethanol, $1 \mathrm{mM} \mathrm{PMSF}$, 5\% glycerol) and the pellets were resuspended in $30 \mathrm{ml}$ of the same buffer. Cells were lysed using a HTU DIGI-F Press (18,000 p.s.i., 138,000 kPa, two passes, G. Heinemann, Germany). After lysis, the crude extracts were centrifuged at $41,000 x$ $g$ for $30 \mathrm{~min}$ at $4^{\circ} \mathrm{C}$, and the RNA polymerase was purified from the supernatant via the His-tagged RpoC as described (Qi and Hulett, 1998). The RNA polymerase-containing fractions were pooled and further purified by size exclusion chromatography. For this purpose, the complex was applied onto a HiLoad 16/600 Superdex 200 column (GE Healthcare) in buffer P. The buffer was filtered (0.2 $\mathrm{m}$ filters) prior to protein separation on an Äkta Purifier (GE Healthcare). The fractions containing RNA polymerase were pooled and dialyzed against RNA polymerase storage buffer (50 $\mathrm{mM}$ Tris- $\mathrm{HCl}$, pH 8.0, $3 \mathrm{mM} \beta$-mercaptoethanol, $0.15 \mathrm{M} \mathrm{NaCl}, 50 \%$ glycerol, 1:1,000). The purified RNA polymerase was stored at $-20^{\circ} \mathrm{C}$.

The housekeeping sigma factor $\sigma^{A}$ was overproduced from plasmid pCD2 (Chang and Doi, 1990) and purified as described (Juang and Helmann, 1994).

\section{In vitro transcription assays}

Multiple round transcription assays were performed as described previously (Wiedermannová et al., 2014), unless stated otherwise. Initiation competent RNA polymerase was reconstituted using the core enzyme and saturating concentration of $\sigma^{A}$ in dilution buffer $(50 \mathrm{mM}$ Tris- $\mathrm{HCl}, \mathrm{pH} 8.0,0.1 \mathrm{M} \mathrm{NaCl}, 50 \%$ glycerol) for $10 \mathrm{~min}$ at $30^{\circ} \mathrm{C}$. Assays were carried out in $10 \mu \mathrm{l}$ with 64 nM RNA polymerase holoenzyme and 100 ng plasmid DNA templates in transcription buffer containing $40 \mathrm{mM}$ Tris- $\mathrm{HCl}$ (pH 8.0), $10 \mathrm{mM} \mathrm{MgCl}, 1 \mathrm{mM}$ dithiothreitol (DTT), $0.1 \mathrm{mg} / \mathrm{ml}$

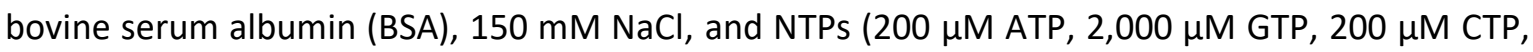
$10 \mu \mathrm{M}$ UTP plus $2 \mu \mathrm{M}$ of radiolabeled [ $\left.\left.\alpha{ }^{-32} \mathrm{P}\right]-\mathrm{UTP}\right)$. The samples were preheated for $10 \mathrm{~min}$ at $37^{\circ} \mathrm{C}$. The reaction was started by the addition of RNA polymerase and allowed to proceed for 20 min (30 min in the case of iNTP-sensing experiments) at $37^{\circ} \mathrm{C}$. Subsequently, the reaction was stopped by the addition of $10 \mu$ of formamide stop solution (95\% formamide, $20 \mathrm{mM}$ EDTA, pH 8.0). The samples were loaded onto $7 \mathrm{M}$ urea-7\% polyacrylamide gels. The gels were dried and 
exposed to Fuji MS phosphor storage screens, scanned with a Molecular Imager FX (BIORAD) and analyzed with Quantity One program (BIORAD).

\section{Transcriptome analysis}

Cells were grown in $\mathrm{LB}$ medium at $37^{\circ} \mathrm{C}$ to an $\mathrm{OD}_{600}$ of 0.5 to $0.6 .5 \mathrm{ml}$ samples of the cultures were added to $10 \mathrm{ml}$ RNA-protect (Qiagen) and allowed to incubate for 5 minutes at room temperature, followed by centrifugation at $5,000 \times \mathrm{g}$ for $10 \mathrm{~min}$ at $4^{\circ} \mathrm{C}$. Pellets were quickly frozen in liquid nitrogen and stored at $-80^{\circ} \mathrm{C}$. A total of three independent biological replicates were included. The harvested pellets were resuspended in $800 \mu \mathrm{l}$ RLT buffer (RNeasy Mini Kit, Qiagen) with $\beta$-mercaptoethanol $(10 \mu \mathrm{l} / \mathrm{ml})$ and cell lysis was performed using a laboratory ball mill. Subsequently $400 \mu \mathrm{l}$ RLT buffer with $\beta$-mercaptoethanol $(10 \mu \mathrm{l} / \mathrm{ml})$ and 1,200 $\mu \mathrm{l} 96 \%[\mathrm{v} / \mathrm{v}$ ] ethanol were added. For RNA isolation, the RNeasy Mini Kit (Qiagen) was used as recommended by the manufacturer, but instead of RW1 buffer RWT buffer (Qiagen) was used to facilitate the isolation of RNAs smaller $200 \mathrm{nt}$. To determine the RNA integrity number (RIN) the isolated RNA was run on an Agilent Bioanalyzer 2100 using an Agilent RNA 6000 Nano Kit as recommended by the manufacturer (Agilent Technologies, Waldbronn, Germany). Remaining genomic DNA was removed by digesting with TURBO DNase (Invitrogen, ThermoFischer Scientific, Paisley, United Kingdom). The Pan-Prokaryozes riboPOOL kit v1 (siTOOLS BIOTECH, Planegg/Martinsried, Germany) was used to reduce the amount of rRNA-derived sequences. For sequencing, the strand-specific cDNA libraries were constructed with a NEBNext Ultra II directional RNA library preparation kit for Illumina (New England BioLabs, Frankfurt am Main, Germany). To assess quality and size of the libraries, samples were run on an Agilent Bioanalyzer 2100 using an Agilent High Sensitivity DNA Kit (Agilent Technologies, Waldbronn, Germany). Concentration of the libraries were determined using the Qubit ${ }^{\circledR}$ dsDNA HS Assay Kit as recommended by the manufacturer (Life Technologies $\mathrm{GmbH}$, Darmstadt, Germany). Sequencing was performed by using the HiSeq4000 instrument (Illumina Inc., San Diego, CA, USA) using the HiSeq 3000/4000 SR Cluster Kit for cluster generation and the HiSeq 3000/4000 SBS Kit (50 cycles) for sequencing in the single-end mode and running $1 \times 50$ cycles. Between 12.623 .708 and 16.865 .134 raw reads were generated for the samples. For quality filtering and removing of remaining adaptor sequences, Trimmomatic-0.39 (Bolger et al., 2014) and a cutoff phred-33 score of 15 were used. The mapping of the remaining sequences was performed with the Bowtie (version 2) program (Langmead and Salzberg, 2012) using the implemented end-to-end mode, which requires that the entire read aligns from one end to the other. First, surviving reads were mapped against a database consisting of tRNA and rRNA sequences of $B$. subtilis 168 and unaligned reads were 
subsequently mapped against the genome of $B$. subtilis 168. Differential expression analyses were performed with the BaySeq program (Mortazavi et al., 2008). Genes with fold change in expression of $\geq 2.0$ or $\leq-2.0$, a likelihood value of $\geq 0.9$, and an adjusted $P$ value of $\leq 0.05$ (the $P$ value was corrected by the false discovery rate [FDR] on the basis of the Benjamini-Hochberg procedure) were considered differentially expressed. The raw reads have been deposited in the National Center for Biotechnology Information's (NCBI) Sequence Read Archive (SRA) under accession no. SRP274247. Functional and regulation information on the differentially expressed genes was obtained from the SubtiWiki database (Zhu and Stülke, 2018).

\section{Model for subunit composition of RNA polymerase}

To test whether the duplication of RNA polymerase core genes can result in incomplete RNA polymerase complexes, a model for complex composition was built based on the following assumptions: (i) Every core RNA polymerase will bind a copy of each component that is available in excess of core. (ii) Other components are allocated to the core RNA polymerases randomly and independently of each other (with exception of $\sigma^{A}$ and NusA). (iii) The probability that such a subunit or transcription factor is associated with core RNA polymerase is estimated by the ratio of the number of molecules of that subunit to the number of cores. The latter ratios are calculated from proteomic mass fractions (Reuß et al., 2017) and the numbers of amino acids in the different proteins. The amount of core RNA polymerase is estimated by the $\beta$ subunit (the $\alpha$ subunit is present at approximately 2:1 ratio as expected from the stoichiometry of core, $\beta^{\prime}$ is slightly in excess of the other two subunits in these data) (Reuß et al., 2017). $\sigma^{A}$ and NusA are treated as binding subsequently during the initiation and elongation stage of transcription with NusA replacing $\sigma^{A}$ during the transition to elongation, thus their numbers are added. The probabilities for core RNA polymerase to form specific complexes are then obtained by combinatorial multiplication of these probabilities.

\section{Acknowledgements}

We are grateful to Alžbeta Rabatinová, Victoria Keidel, Janek Meißner and Fabian Commichau for providing strains and to Gabriele Beyer, Melanie Heinemann and Sarah Teresa Schüßler for technical support. We thank Jonas Jennrich for helpful discussions. 


\section{The YtrBCDEF ABC transporter is involved in the control of social activities in Bacillus subtilis}

The results of this chapter are published in the following pre-print:

Benda, M., Schulz, L. M., Rismondo, J. and Stülke, J. (2020) The YtrBCDEF ABC transporter is involved in the control of social activities in Bacillus subtilis. bioRxiv 2020.07.24.219923.

Author contribution:

MB constructed the strains. MB performed transformation experiments, biofilm assay and fluorescence microscopy. LMS performed electron microscopy. MB, LMS, JR and JS analyzed the data. MB and JS designed the study and wrote the manuscript. 


\section{Abstract}

Bacillus subtilis develops genetic competence for the uptake of foreign DNA when cells enter the stationary phase and a high cell density is reached. These signals are integrated by the competence transcription factor ComK which is subject to transcriptional, post-transcriptional and post-translational regulation. Many proteins are involved the development of competence, both to control ComK activity and to mediate DNA uptake. However, for many proteins, the precise function they play in competence development is unknown. In this study, we have tested whether proteins required for genetic transformation play a role in the activation of ComK or rather downstream of competence gene expression. While these possibilities could be distinguished for most of the tested factors, two proteins (PNPase and the transcription factor YtrA) are required both for full ComK activity and for the downstream processes of DNA uptake and integration. Further analyses of the role of the transcription factor YtrA for the competence development revealed that the constitutive expression of the YtrBCDEF $A B C$ transporter in the $y \operatorname{trA}$ mutant causes the loss of genetic competence. Moreover, constitutive expression of this $A B C$ transporter also interferes with biofilm formation. Since the $y \operatorname{trGABCDEF}$ operon is induced by cell walltargeting antibiotics, we tested the cell wall properties upon overexpression of the $A B C$ transporter and observed an increased thickness of the cell wall. The composition and properties of the cell wall are important for competence development and biofilm formation, suggesting, that the increased cell wall thickness as a result of YtrBCDEF overexpression causes the observed phenotypes. 


\section{Introduction}

The gram-positive model bacterium Bacillus subtilis has evolved many different ways to survive harsh environmental conditions, i. e. it can form highly resistant spores, secrete toxins to kill and cannibalize neighboring cells, form resistant macroscopic biofilms or become competent for transformation (reviewed in (López and Kolter, 2010).

Development of genetic competence is a strategy, which allows bacterial cells to take up foreign DNA from the environment in order to extend the genetic variability of the population. Competence is developed during the transition from exponential to stationary phase of growth as a response to increased cell density and nutrient limitation. In B. subtilis, genetic competence is developed in a bistable manner, meaning that only about $10-20 \%$ of the cells of a population change their physiological characteristics and become competent for transformation, leaving the rest of the population non-competent in an all or nothing scenario (Haijema et al., 2001; Maamar and Dubnau, 2005). Whether a specific cell becomes competent or not depends on the level of the master regulator ComK (van Sinderen et al., 1995), whose cellular amount is tightly controlled by a complex network of regulators acting on the transcriptional, post-transcriptional as well as on post-translational levels (for a detailed overview see (Maier, 2020).

Transcription of the comK gene is controlled by three repressor proteins, Rok, CodY, and AbrB (Serror and Sonenshein, 1996; Hoa et al., 2002; Hamoen et al., 2003a), moreover, comK transcription is activated by the transcriptional regulator DegU (Hamoen et al., 2000). Another important player for comK regulation is SpoOA-P, which controls the levels of the AbrB repressor and additionally supports activation of ComK expression by antagonizing Rok (Hahn et al., 1995; Mirouze et al., 2012). The presence of phosphorylated Spo0A directly links competence to other lifestyles, since Spo0A-P is also involved in pathways leading to sporulation or biofilm formation (Aguilar et al., 2010). When ComK expression reaches a certain threshold, it binds its own promoter region to further increase its own expression, thereby creating a positive feedback loop which leads to full activation of competence (Maamar and Dubnau, 2005; Smits et al., 2005).

ComK levels are also controlled post-transcriptionally by the Kre protein, which destabilizes the comK mRNA (Gamba et al., 2015). Post-translational regulation is achieved through the adapter protein MecA, which sequesters ComK and directs it towards degradation by the ClpCP protease (Turgay et al., 1998). During competence, this degradation is prevented by a small protein, ComS, that is expressed in response to quorum sensing (Nakano et al., 1991).

ComK activates expression of more than 100 genes (Berka et al., 2002; Hamoen et al., 2002; Ogura et al., 2002; Boonstra et al., 2020). Whereas a clear role in competence development 
has been assigned to many of the ComK regulon members, the roles of some ComK-dependent genes remain unclear. Similarly, many single deletion mutant strains were identified as competence deficient, and for many of them the reasons for this deficiency are obvious. However, there are still many single deletion mutants deficient in genetic competence, in which the reason for the loss of competence remains unknown. Typical examples for this are various RNases, namely RNase Y, RNase J1, PNPase or nanoRNase A (Luttinger et al., 1996; Figaro et al., 2013; our unpublished results). Recently, a library of single knock outs of $B$. subtilis genes was screened for various phenotypes, including competence development (Koo et al., 2017). This screen revealed 21 mutants with completely abolished competence. Out of those, 16 are known to be involved in the control of the ComK master regulator, DNA uptake or genetic recombination. However, in case of the other 5 competence-defective strains the logical link to competence is not obvious.

Here, we have focused on some of these factors to investigate their role in genetic competence in more detail. We took advantage of the fact that artificial overexpression of ComK and ComS significantly increases transformation efficiency independently of traditional ComK and ComS regulations (Rahmer et al., 2015). This allows the identification of genes that are involved in competence development due to a function in ComK expression or for other specific reasons downstream of ComK activity. We identified the $y \operatorname{trGABCDEF}$ operon as an important player for B. subtilis differentiation, since its constitutive expression does not only completely block competence by a so far unknown mechanism, but also affects the proper development of other lifestyles of $B$. subtilis. We discuss the role of thicker cell walls upon overexpression of the proteins encoded by the $y \operatorname{trGABCDEF}$ operon as the reason for competence and biofilm defects.

\section{Results}

\section{ComK-dependent and-independent functions of proteins required for the development}

\section{of genetic competence}

Genetic work with $B$. subtilis is facilitated by the development of genetic competence, a process that depends on a large number of factors. While the specific contribution of many proteins to the development of competence is well understood, this requirement has not been studied for many other factors. In particular, several RNases (RNase Y, RNase J1, PNPase and nanoRNase A) are required for competence, and the corresponding mutants have lost the ability to be become naturally competent (Luttinger et al., 1996; Figaro et al., 2013; our unpublished 
results). We are interested in the reasons for the loss of competence in these mutant strains, as well as in other single gene deletion mutants which are impaired in the development of natural competence for unknown reasons (Koo et al., 2017). Therefore, we first tested the roles of the aforementioned RNases (encoded by the $r n y, r n j A$, $p n p A$, and $n r n A$ genes) as well as of the transcription elongation factor GreA, the metalloprotease FtsH and the transcription factor YtrA (Koo et al., 2017) for the development of genetic competence. For this purpose, we compared the transformation efficiencies of the corresponding mutant strains to that of a wild type strain. We have included two controls to all experiments, i. e. comEC and degU mutants. Both mutants have completely lost genetic competence, however for different reasons. The ComEC protein is directly responsible for the transport of the DNA molecule across the cytoplasmic membrane. Loss of ComEC blocks competence, but it should not affect the global regulation of competence development and expression of other competence factors (Draskovic and Dubnau, 2005). In contrast, DegU is a transcription factor required for the expression of the key regulator of competence, ComK, and thus indirectly also for the expression of all other competence genes (Hamoen et al., 2000; Shimane and Ogura, 2004). Our analysis confirmed the significant decrease in transformation efficiency for all tested strains (see Table 2). For five out of the seven strains, as well as the two control strains competence was abolished completely, whereas transformation of strains GP2155 ( $\Delta n r n A)$ and GP1748 ( $\Delta p n p A)$ was possible, but severely impaired as compared to the wild type strain. This result confirms the implication of these genes in the development of genetic competence.

The proteins that are required for genetic competence might play a more general role in the control of expression of the competence regulon (as known for the regulators that govern ComK expression and stability, e. g. the control protein DegU), or they may have a more specific role in competence development such as the control protein ComEC. To distinguish between these possibilities, we introduced the mutations into a strain that allows inducible overexpression of the comK and comS genes. The overexpression of comK and comS allows transformation in rich medium and hence facilitates the transformation of some competence mutants (Rahmer et al., 2015). For this purpose, we first constructed strains that contain mannitol inducible comK and coms genes fused to resistance cassettes (GP2618 and GP2620, for details see Experimental procedures). Subsequently, we deleted our target genes in this genetic background and assayed transformation efficiency after induction of comKS expression (for details see Experimental procedures). In contrast to the strain with wild type comK expression, the transformation efficiency of the degU mutant was now similar to the isogenic wild type strain. This suggests that DegU affects competence only by its role in comK expression and that DegU is no longer required in the strain with inducible comKS expression. In contrast, the comEC mutant was even in this case 
completely non-competent, reflecting the role of the ComEC protein in DNA uptake (see Table 2). Of the tested strains, only the $n r n A$ mutant showed a transformation efficiency similar to that of the isogenic control strain with inducible comKS expression. This observation suggests that nanoRNase A might be involved in the control of comK expression. In contrast, the fts $\mathrm{H}$, greA, rny and rnjA mutants did not show any transformants even upon comKS overexpression, indicating that the corresponding proteins act downstream of comK expression. Finally, we have observed a small but reproducible restoration of competence in case of the pnpA and ytrA mutants. This finding is particularly striking in the case of the $y \operatorname{trA}$ mutant, since this strain did not yield a single transformant in the 168 background (see Table 2). However, the low number of transformants obtained with pnpA and ytrA mutants as compared to the isogenic wild type strain suggests that PNPase and the YtrA transcription factor play as well a role downstream of comK.

Table 2: Effect of gene deletions on the development of genetic competence in dependence of the competence transcription factor Comk ${ }^{\mathrm{a}}$

\begin{tabular}{lcc}
\hline & \multicolumn{1}{c}{ Wild type } & $P_{m+1 A}$-ComKS \\
Mutant & \multicolumn{1}{c}{ Colonies per $\mu$ o of DNA } \\
\hline Wild type & $138,600 \pm 17,006$ & $47,952 \pm 8,854$ \\
$\Delta$ degU & $0 \pm 0$ & $60,853 \pm 13,693$ \\
$\Delta$ comEC & $0 \pm 0$ & $0 \pm 0$ \\
$\Delta n r n A$ & $1,689 \pm 316$ & $34,933 \pm 6,378$ \\
$\Delta$ ftsH & $0 \pm 0$ & $0 \pm 0$ \\
$\Delta$ greA & $0 \pm 0$ & $0 \pm 0$ \\
$\Delta r n y$ & $0 \pm 0$ & $0 \pm 0$ \\
$\Delta r n j A$ & $0 \pm 0$ & $0 \pm 0$ \\
$\Delta$ pnpA & $17 \pm 6$ & $293 \pm 19$ \\
$\Delta y \operatorname{trA}$ & $0 \pm 0$ & $467 \pm 278$ \\
\hline
\end{tabular}

a Cells were transformed with chromosomal DNA of strain GP1152 harboring a tetracycline resistance marker as described in Experimental procedures.

ComK activates transcription of many competence genes including comG (van Sinderen et al., 1995). Therefore, as a complementary approach to further verify the results shown above, we decided to assess ComK activity using a fusion of the comG promoter to a promoterless GFP reporter gene (Gamba et al., 2015). For this purpose, we deleted the selected genes in the background of strain GP2630 containing the $\mathrm{P}_{\text {comG }}-g f p$ construct. We grew the cells in competence inducing medium using the two-step protocol as we did for the initial transformation experiment. At the time point, when DNA would be added to the cells during the transformation procedure, we assessed com $G$ promoter activity in the cells using fluorescence microscopy. Since expression of ComK and thus also activation of competence takes place only in sub-population of cells (Smits et al., 2005), we determined the ratio of $g f p$ expressing cells as an indication of ComK activity for 
each of the strains (see Table 3). Since RNase mutants tend to form chains, thus making it difficult to study florescence in individual cells, we did not include the RNase mutants for this analysis.

In the wild type strain GP2630, about $20 \%$ of the cells expressed GFP, and similar numbers were obtained for the control strain lacking ComEC, which is not impaired in comK and subsequent comG expression. In contrast, the control strain lacking DegU showed decreased amount of GFP expressing cells as compared to the wild type, which reflects the role of DegU in the activation of comK expression. In agreement with our previous finding that nanoRNase A affects ComK activity, only about $3 \%$ of $n r n A$ mutant cells showed expression from $\mathrm{P}_{\text {comG }}-g f p$. For the $\mathrm{ftsH}$ mutant, we did not find any single cell expressing GFP. This is striking since our previous results suggested that ComK expression is not the cause of competence deficiency in this case. For the strain lacking GreA, we observed similar rates of GFP expressing cells as in the wild-type strain, indicating that ComK activation is not the problem that causes loss of competence. Finally, we have observed significantly decreased ratio of GFP producing cells in case of the ytrA deletion mutant.

Table 3: Effect of gene deletions on the activity of the competence transcription factor ComK as studied by the percentage of cells expressing a $\mathrm{P}_{\text {comG }}$-gfp transcriptional fusion ${ }^{\mathrm{a}}$.

\begin{tabular}{lc}
\hline Mutant & GFP expressing cells \\
\hline Wild type & $21.1 \% \pm 0,8 \%$ \\
$\Delta$ degU & $8.4 \% \pm 4.1 \%$ \\
$\Delta$ comEC & $21.1 \% \pm 0.3 \%$ \\
$\Delta n r n A$ & $3.5 \% \pm 1.0 \%$ \\
$\Delta f t s H$ & $0 \% \pm 0 \%$ \\
$\Delta g r e A$ & $17.9 \% \pm 1.3 \%$ \\
$\Delta y \operatorname{tr} A$ & $2.2 \% \pm 0.6 \%$ \\
\hline
\end{tabular}

${ }^{\text {a }}$ Strains harboring the $\mathrm{P}_{\text {com }}-\mathrm{gfp}$ construct were grown in competence inducing medium and the percentage of GFP expressing cells was determined. Data were collected from three pictures originated from at least two independent growth replicates.

Taken together we have discovered that nrnA coding for nanoRNase A (Mechold et al., 2007) plays a so far undiscovered role in the regulation of comK. In contrast, the GreA transcription elongation factor is required for competence development in steps downstream of comK expression. FtsH and YtrA seem to play a dual role in the development of genetic competence. On one hand, they are both required for ComK activity but on the other hand, they have a ComK-independent function. The ytrA gene encodes a transcription factor with a poorly studied physiological function (Salzberg et al., 2011). Therefore, we focused our further work on understanding the role of this gene in development of genetic competence. 


\section{Overexpression of the YtrBCDEF ABC transporter inhibits genetic competence}

The ytrA gene encodes a negative transcription regulator of the GntR family, which binds to the inverted repeat sequence AGTGTA-13bp-TACACT (Salzberg et al., 2011). In the B. subtilis genome, this sequence is present in front of two operons, its own operon ytrGABCDEFG and $y w o B C D$. The deletion of $y \operatorname{trA}$ leads to an overexpression of these two operons (Salzberg et al., 2011). It is tempting to speculate that overexpression of one of these operons is the cause for the loss of competence in the ytrA mutant. To test this hypothesis, we constructed strain GP2646, which lacks the complete $y \operatorname{trGABCDEF}$ operon. Next, we assayed the genetic competence of this strain. This revealed that although deletion of ytrA fully blocks genetic competence, the strain lacking the whole operon is transformable in similar rates as the wild type strain 168 (see Table 4). We conclude that overexpression of the $y \operatorname{trGABCDEF}$ operon causes the loss of competence in the ytrA mutant strain. In addition, we tested ComK activity in the mutant lacking the operon, using the expression of the $\mathrm{P}_{\text {comG }}-\mathrm{gfp}$ fusion as a readout. As observed for the wild type, about $20 \%$ of the mutant cells expressed comG, indicating that ComK is fully active in the mutant, and that the reduced activity in the ytrA mutant results from the overexpression of the operon (data not shown). Initially we also attempted deleting the $y w O B C D$ operon, however we failed to construct such a strain in several experiments. As we have already discovered that the overexpression of the $y$ tr operon causes the loss of competence in the ytrA mutant, we decided not to continue with this second YtrA-controlled operon.

The $y$ tr operon consist of seven genes (see Fig. 12A). Five proteins encoded by this operon (YtrB, YtrC, YtrD, YtrE and YtrF) are components of a putative ABC transporter (see Fig. 12B), which was suggested to play a role in acetoin utilization (Quentin et al., 1999; Yoshida et al., 2000). $Y \operatorname{tr} B$ and $Y \operatorname{trE}$ are supposed to be the nucleotide binding domains, $Y \operatorname{tr} C$ and $Y \operatorname{tr} D$ the membrane spanning domains and YtrF the substrate binding protein. Finally, another open reading frame called $y \operatorname{tr} G$, encodes a peptide of 45 amino acids which is unlikely to be part of the ABC transporter (Salzberg et al., 2011). The expression of the ytr operon is usually kept low due to transcriptional repression exerted by YtrA. This repression is naturally relieved only in response to several lipid II-binding antibiotics or during cold-shock (Beckering et al., 2002; Salzberg et al., 2011; Wenzel et al., 2012). 
A

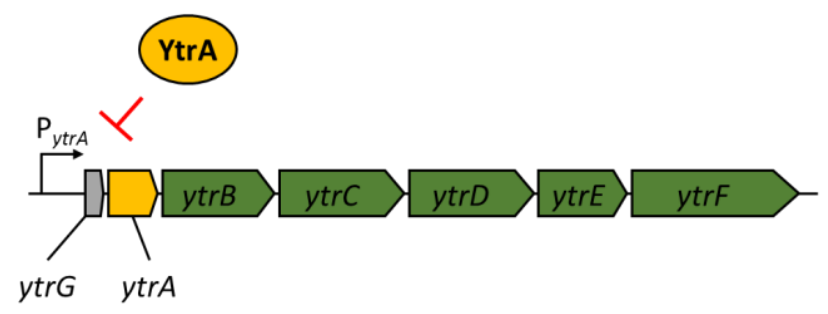

B

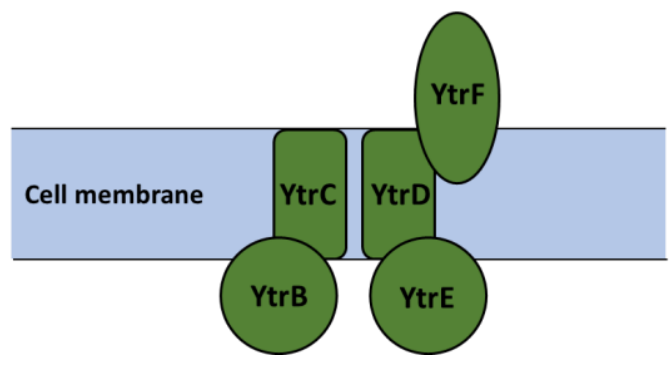

Figure 12: Genetic organization of the $y \operatorname{trGABCDEF}$ operon and organization of the putative $A B C$ transporter

(A) Reading frames are depicted as arrows with respective gene names. Green arrows indicate proteins suggested to form the $A B C$ transporter; the yellow arrow indicates the gene coding for the repressor YtrA and the grey arrow indicates the small open reading frame called $y$ trG. The map was constructed based on information provided in Salzberg et al. (2011) (B) Organization of the putative ABC transporter YtrBCDEF as suggested by Yoshida et al. (2000). YtrB and YtrE are nucleotide binding proteins, YtrC and YtrD membrane spanning proteins and YtrF is a solute binding protein. The role and localization of the YtrG peptide remain elusive.

To test the involvement of the individual components of the putative YtrBCDEF $A B C$ transporter in the development of genetic competence, we constructed double mutants of ytrA together with each one of the other genes of the operon, i.e. $y \operatorname{tr} B, y \operatorname{trC}, y \operatorname{trD}, y \operatorname{trE}$ and $y \operatorname{trF}$. The results (see Table 4) revealed that most of the double mutants are deficient in genetic transformation, as observed for the single ytrA mutant GP2647. However, strain GP3187 with deletions of $y \operatorname{trA}$ and $y \operatorname{trF}$ but still overexpressing all the other parts of the transporter, had partially restored competence. We conclude that the YtrF protein is the major player for the loss of competence in the overexpressing strain.

To further test the role of YtrF overexpression for the loss of competence, we used two different approaches. First, we constructed a strain with artificial overexpression of ytrF from a xylose inducible promoter (GP3197) and second, we created a strain with deletion of all other components (ytrGABCDEF) of the operon, leaving only constitutively expressed $y \operatorname{trF}$ (GP3186). In contrast to our expectations, competence was not blocked in any of the two strains, suggesting that increased presence of YtrF protein alone is not enough to block the competence and that YtrF might need assistance from the other proteins of the putative transporter for its full action/proper localization. The ytr operon encodes two putative nucleotide binding proteins (YtrB and $Y \operatorname{trE})$ and two putative membrane spanning proteins ( $Y \operatorname{trC}, Y \operatorname{trD})$, whereas $Y \operatorname{trF}$ is the only solute binding protein that interacts with the transmembrane proteins. Therefore, we hypothesized that YtrF overexpression might only block genetic competence if the protein is properly localized in the membrane via YtrC and YtrD. To check this possibility, we constructed strains GP3206 and GP3213 lacking YtrA and the nucleotide binding proteins or the membrane proteins, respectively, and tested their transformability. Strain GP3206 showed very few 
transformants, suggesting that the presence of nucleotide binding proteins is not important to block competence. In contrast, strain GP3213 gave rise to many transformants. We thus conclude that the overexpression of the solute binding protein YtrF in conjunction with the membrane proteins $Y \operatorname{trC}$ and $Y \operatorname{trD}$ is responsible for the block of competence indicating that indeed the proper function of $Y \operatorname{trF}$, which depends on $Y \operatorname{trC}$ and $Y \operatorname{trD}$, is crucial for the phenotype.

Table 4: Effect of gene deletions in the ytrGABCDEF operon on the development of genetic competence ${ }^{a}$.

\begin{tabular}{lc}
\hline Mutant & Colonies per $\mu \mathrm{g}$ of DNA \\
\hline Wild type & $138,600 \pm 17,006$ \\
$\Delta y \operatorname{trGABCDEF}$ & $114,733 \pm 14,408$ \\
$\Delta y \operatorname{trA}$ & $0 \pm 0$ \\
$\Delta y \operatorname{tr} A B$ & $0 \pm 0$ \\
$\Delta y \operatorname{tr} A C$ & $0 \pm 0$ \\
$\Delta y \operatorname{tr} A D$ & $24 \pm 2$ \\
$\Delta y \operatorname{tr} A E$ & $137 \pm 51$ \\
$\Delta y \operatorname{tr} A F$ & $10,180 \pm 549$ \\
P $_{\text {xy- }-y \operatorname{trF}}$ & $137,533 \pm 26,595$ \\
$\Delta y \operatorname{tr} G A B C D E$ & $108,467 \pm 14,836$ \\
$\Delta y \operatorname{tr} A B E$ & $309 \pm 88$ \\
$\Delta y \operatorname{tr} A C D$ & $45,467 \pm 10,799$ \\
\hline
\end{tabular}

a Cells were transformed with chromosomal DNA of strain GP1152 harboring a tetracycline resistance marker as described in Experimental procedures

\section{Overexpression of the ytrGABCDEF operon leads to defect in biofilm formation}

B. subtilis can employ various lifestyles which are tightly interconnected through regulatory proteins (López et al., 2009). Therefore, we anticipated that the overexpression of YtrF might also affect other lifestyles of $B$. subtilis. Indeed, it was previously shown that the ytrA mutant has a reduced sporulation efficiency (Koo et al., 2017). We thus decided to examine the effect of the $y$ trA deletion on biofilm formation. To that end, we first deleted the ytrA gene or the whole $y \operatorname{trGABCDEF}$ operon from the biofilm-forming strain DK1042 (Konkol et al., 2013). We then tested the biofilm formation of the resulting strains on biofilm inducing MSgg agar (Branda et al., 2001). As expected, the wild type strain DK1042 formed structured colonies that are indicative of biofilm formation. In contrast, the negative control GP2559 (a ymdB mutant that is known to be defective in biofilm formation, Kampf et al., 2018) formed completely smooth colonies. The biofilm formed by the ytrA mutant GP3212 was less structured, more translucent and with only some tiny wrinkles on its surface, indicating that biofilm formation was inhibited but not fully abolished upon loss of YtrA. In contrast, strain GP3207 lacking the complete ytrGABCDEF operon 
formed biofilm indistinguishable from the one of the parental strain DK1042 (see Fig. 13). This observation suggests that overexpression of components of the $Y \operatorname{tr} A B C$ transporter interferes with biofilm formation.

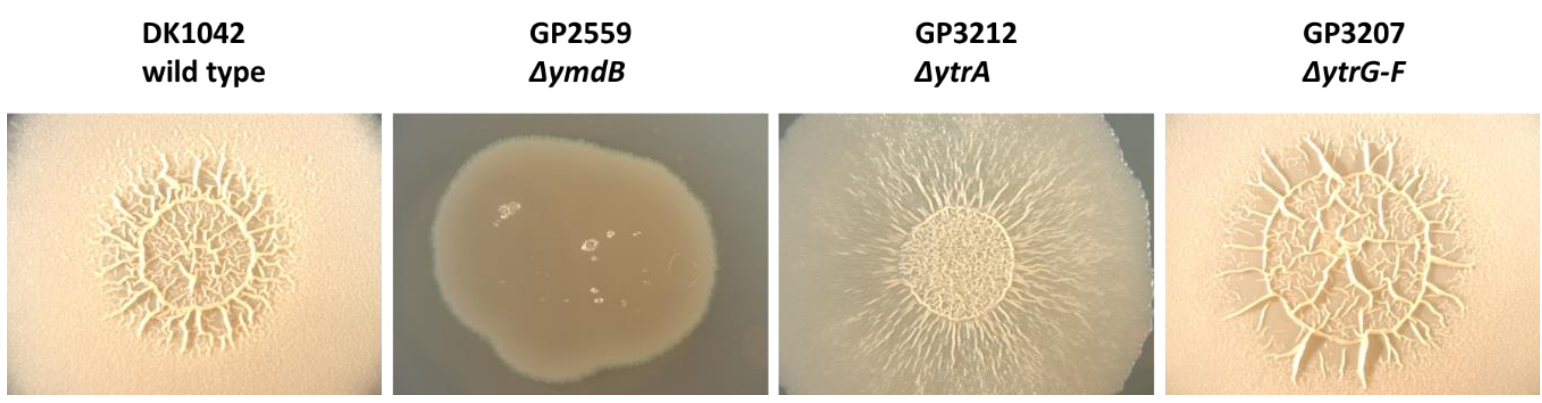

Figure 13: Biofilm formation is affected by the ytrA deletion

Biofilm formation was examined in the wild type strain DK1042 and respective deletion mutants of $y m d B$ (G2559), ytrA (GP3212) and ytrGABCDEF (GP3207). The biofilm assay was performed on MSgg agar plates as described in Experimental procedures. The plates were incubated for 3 days at $30^{\circ} \mathrm{C}$. All images were taken at the same magnification.

\section{Overexpression of the ytr operon increases cell wall thickness}

In previous experiments, we have shown that the expression of the ytr operon interferes with the development of genetic competence and biofilm formation due to the activity of the solute binding protein YtrF. However, it remains unclear why competence and biofilm formation are abolished. The ytr operon is repressed under standard conditions by the YtrA transcription regulator and this repression is naturally relieved only upon exposure to very specific stress conditions, mainly in response to cell wall targeting antibiotics and cold shock (Cao et al., 2002; Beckering et al., 2002; Mascher et al., 2003; Salzberg et al., 2011; Nicolas et al., 2012; Wenzel et al., 2012). The possible link between antibiotic resistance, genetic competence, and biofilm formation is not apparent, however, cell wall properties might provide an answer. Indeed, it has been shown that wall teichoic acids, the uppermost layer of the cell wall, are important for DNA binding during the process of transformation and biofilm formation (Bucher et al., 2015; Zhu et al., 2018; Mirouze et al., 2018).

To test the hypothesis that overexpression of the putative $A B C$ transporter encoded by the $y \operatorname{trGABCDEF}$ operon affects cell wall properties of the $B$. subtilis cells, we decided to compare the cell morphology of the wild type and the $y \operatorname{trA}$ mutant as well as the $y \operatorname{trGABCDEF}$ mutant lacking the complete operon by transmission electron microscopy. While the wild type strain showed an average cell wall thickness of $21 \mathrm{~nm}$, which is agreement with previous studies 
(Beveridge and Murray, 1979), the ytrA (GP2647) mutant showed a significant increase in cell wall thickness with an average of $31 \mathrm{~nm}$. In contrast, such an increase was not observed for the whole operon mutant (GP2646) that had an average cell wall thickness of $23 \mathrm{~nm}$ (see Fig. 14). These observations are in excellent agreement with the hypothesis that the overexpression of the YtrBCDEF $A B C$ transporter affects cell wall properties and thereby genetic competence and biofilm formation.

A
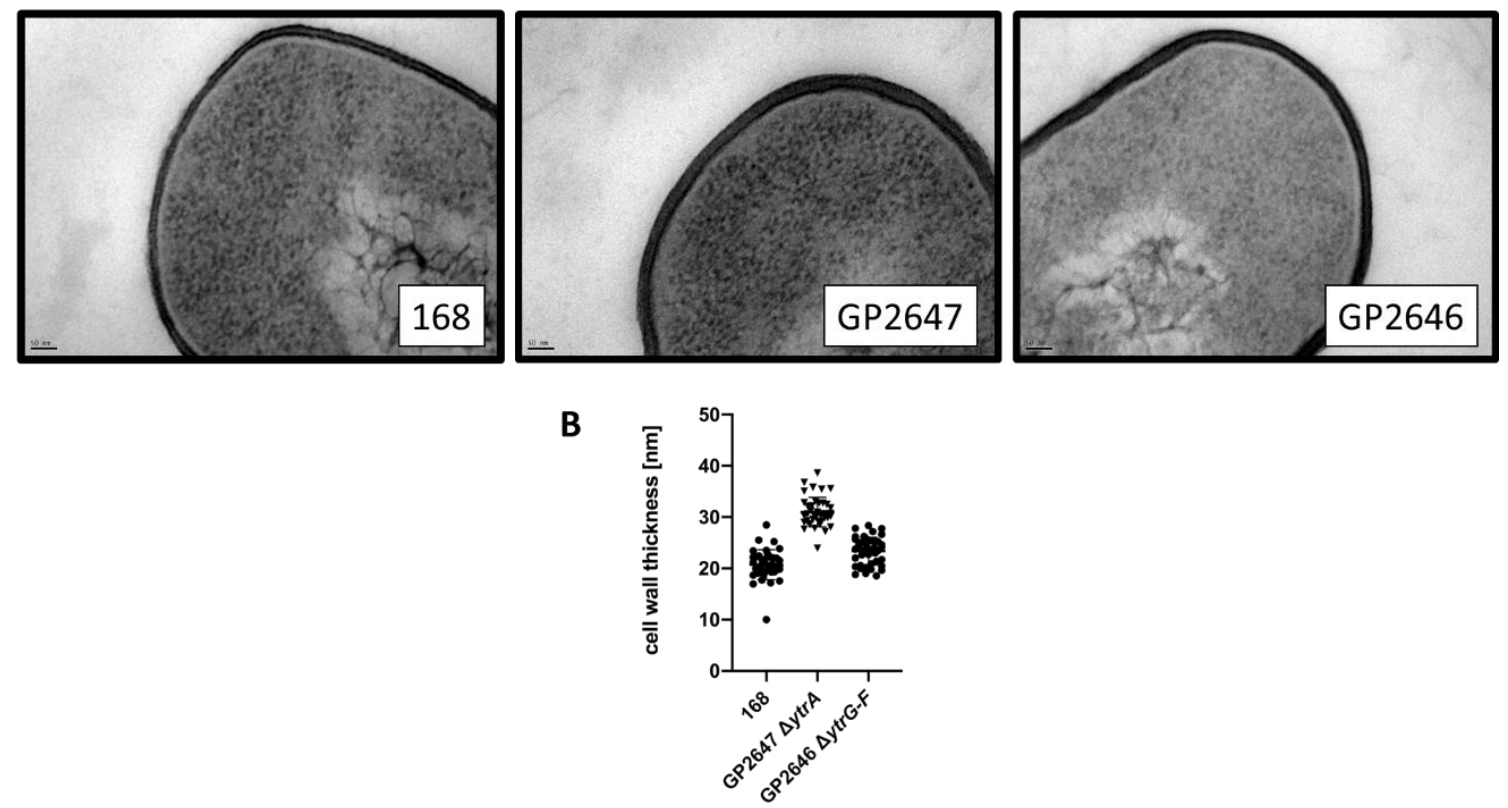

Figure 14: The ytrA mutant has thicker cell walls

(A) Shown are representative transmission electron microscopy images of the wild type strain 168, the ytrA mutant (GP2647) and the whole operon ytrGABCDEF mutant (GP2646). (B) The graph shows the cell wall thickness of 40 individual measurements from two growth replicates as described in Experimental procedures

\section{Discussion}

In this work we have shown that overexpression of the $y \operatorname{trGABCDEF}$ operon, coding for a so far uncharacterized $A B C$ transporter, completely blocks the development of genetic competence and interferes with biofilm formation in $B$. subtilis. This block is mediated by the solute binding protein YtrF in cooperation with at least one membrane spanning protein (YtrC or YtrD) that are required for correct function of YtrF. The overexpression of the YtrBCDEF ABC transporter is the reason for the loss of competence of an ytrA regulator mutant that had been observed in a previous genome-wide study (Koo et al., 2017). Based on its expression pattern, the ytr operon was described as a reporter for glycopeptide antibiotics, such as vancomycin or ristocetin (Hutter et al., 2004) and later also for other antibiotics that interfere with the lipid II 
cycle, such as nisin (Wenzel et al., 2012). Whether this induction of ytrGABCDEF expression leads to an increased resistance towards those antibiotics is not clear, but recent results indicate that it does at least in case of nisin (J. Bandow, personal communication).

Based on the partial restoration of genetic competence of the ytrA mutant upon ComKS overexpression, one might expect that the loss of YtrA and the concomitant overexpression of the $A B C$ transporter somehow interferes with competence development upstream of ComK activation. However, competence is developed in an all or nothing scenario, and cells in which the ComK levels reach a certain threshold should become competent (Haijema et al., 2001; Maamar and Dubnau, 2005). Our observation that comKS overexpression restores competence of the ytrA mutant only partially suggests that ComK levels are not the only factor that limits competence of the $y \operatorname{trA}$ mutant. If the $y \operatorname{trA}$ deletion would interfere with ComK activation, one would then expect wild type like competence upon overexpression of ComK which was not the case. Why does ComK then restore the competence at all? The DNA uptake apparatus must be adapted to cell wall thickness in order to ensure that the extracellular DNA can reach the ComG/ComE DNA transport complex. Due to the increased cell wall thickness upon overexpression of the YtrBCDEF ABC transporter, the DNA probably has problems to get in contact with the ComG pili. Overexpression of ComK will then result in the increased production of DNA-binding ComG on the cell surface of all cells of the population (comparing to about $10 \%$ in the wild-type strain transformed with the classical two-step protocol). This would simply increase the probability that foreign DNA reaches the DNA uptake machinery in some cells, which then leads to the appearance of only a few transformants as observed in our study. On the other hand, the results obtained by fluorescence microscopy revealed a decreased transcription from the ComK dependent comG promoter in the ytrA mutant. However, this expression is expected to be wild type-like if the action of YtrBCEDF $A B C$ transporter would not interfere with ComK activity and only block DNA uptake as a result of the remodeled cell wall as suggested above. Again, the disorganized cell wall might be responsible, since ComK expression is induced by the detection of extracellular quorum-sensing signals (both ComXPA and Rap-Phr systems) and this induction depends on the accessibility of the sensor domains for the pheromones which might be impaired in the strain with altered cell wall composition.

In addition to the loss of genetic competence, it was previously shown that the ytrA deletion leads to decreased sporulation efficiency (Koo et al., 2017) and we have shown that it also affects biofilm formation. Considering the changed cell wall properties, this is in agreement with previous studies which showed hampered biofilm formation upon disruption of cell wall biosynthesis (Bucher et al., 2015; Zhu et al., 2018). Taken together, we conclude that the 
overexpression of the YtrBCDEF ABC transporter upon deletion of $y$ trA plays a pleiotropic role in the control of alternative lifestyles of $B$. subtilis.

Our results demonstrate that the YtrBCDEF ABC transporter is involved in the control of cell wall homeostasis, but it is not yet clear how this is achieved. An easy explanation would be that the system exports molecules necessary for cell wall synthesis, however, based on the presence of the solute binding protein YtrF and on the critical role of this protein in preventing genetic competence, it can be assumed that the $A B C$ transporter rather acts as an importer. However, YtrBCDEF may not act as a transporter at all and simply modulate the activity of other enzymes that participate in cell wall metabolism. Strikingly, YtrF is a member of the same protein family as FtsX, which is known to activate the cell wall hydrolase CwlO (Meisner et al., 2013). Future work will need to address the precise mechanism by which the YtrBCDEF ABC transporter interferes with cell wall synthesis.

\section{Experimental procedures}

\section{Bacterial strains and growth conditions}

The B. subtilis strains used in this study are listed in Table S3. Lysogeny broth (LB, Sambrook et al., 1989) was used to grow E. coli and B. subtilis. When required, media were supplemented with antibiotics at the following concentrations: ampicillin $100 \mathrm{\mu g} \mathrm{ml}^{-1}$ (for E. coli) and chloramphenicol $5 \mathrm{\mu g} \mathrm{ml}^{-1}$, kanamycin $10 \mathrm{\mu g} \mathrm{ml}^{-1}$, spectinomycin $250 \mathrm{\mu g} \mathrm{ml}^{-1}$, tetracycline 12.5 $\mu \mathrm{g} \mathrm{ml} l^{-1}$, and erythromycin $2 \mathrm{ug} \mathrm{ml}^{-1}$ plus lincomycin $25 \mathrm{\mu g} \mathrm{ml}^{-1}$ (for B. subtilis). For agar plates, $15 \mathrm{~g}$ $\mathrm{I}^{-1}$ Bacto agar (Difco) was added.

\section{DNA manipulation and strain construction}

S7 Fusion DNA polymerase (Mobidiag, Espoo, Finland) was used as recommended by the manufacturer. DNA fragments were purified using the QIAquick PCR Purification Kit (Qiagen, Hilden, Germany). DNA sequences were determined by the dideoxy chain termination method (Sambrook et al., 1989). Chromosomal DNA from B. subtilis was isolated using the peqGOLD Bacterial DNA Kit (Peqlab, Erlangen, Germany) and plasmids were purified from E. coli using NucleoSpin Plasmid Kit (Macherey-Nagel, Düren, Germany). Deletion of the degU, comEC, ftsH, greA, $y \operatorname{tr} A, n r n A$, and $y \operatorname{trF}$ genes as well as $y \operatorname{trCD}, y \operatorname{trG}-y \operatorname{tr} E$, and $y \operatorname{trGABCDEF}$ regions was achieved by transformation with PCR products constructed using oligonucleotides (see Table S5) to amplify DNA fragments flanking the target genes and intervening antibiotic resistance cassettes as described previously (Youngman, 1990; Guérout-Fleury et al., 1995; Wach, 1996). The identity of 
the modified genomic regions was verified by DNA sequencing. To construct the strains (GP2618 and GP2620) harbouring the $\mathrm{P}_{m t / A}$-comKS cassette coupled to the antibiotic resistance gene, we have first amplified the $\mathrm{P}_{m t / A}$-comKS from the strain PG10 (Reuß et al., 2017) as well as the resistance genes from pDG646 and pGEM-cat, respectively (Youngman, 1990; Guérout-Fleury et al., 1995) and the genes flanking the intended integration site, i. e. $y v c A$ and his/ from B. subtilis 168. Subsequently, those DNA fragments were fused in another PCR reaction thanks to the overlapping primers. The final product was used to transform B. subtilis 168 . Correct insertion was verified by PCR amplification and sequencing. Markerless deletions of $y \operatorname{tr} B, y \operatorname{trC}, y \operatorname{tr} D$ and $y \operatorname{trE}$ genes were performed using pDR244 plasmid as described (Koo et al., 2017). In short, strains BKE30450, BKE30440, BKE30430 and BKE30420 were transformed with plasmid pDR244 and transformants were selected on LB agar plates supplemented with spectinomycin at $30^{\circ} \mathrm{C}$. Transformants were then streaked on plain LB agar plates and incubated at $42^{\circ} \mathrm{C}$ to cure the plasmid, which contains a thermo-sensitive origin of replication. Single colonies were then screened for spectinomycin and erythromycin/lincomycin sensitivity. Markerless deletion was confirmed by PCR with primers flanking the deletion site. Created strains GP3188, GP3189, GP3190 and GP 3191 were used for subsequent deletion of the $y \operatorname{trA}$ gene. This was done either by transformation with PCR product as described above or by transformation with genomic DNA of the ytrA deletion strain (in case of GP3195 construction). Deletion of the ytrA gene and preservation of selected markerless deletions were confirmed via PCR. To construct GP3206, PCR product containing erythromycin resistance in place of $y \operatorname{tr} A$ and $y \operatorname{tr} B$ genes was amplified from GP3193 and transformed to GP3191.

\section{Transformation of $B$. subtilis strains}

Transformation experiments were conducted based on the two-step protocol as described previously (Kunst and Rapoport, 1995). Briefly, cells were grown at $37^{\circ} \mathrm{C}$ at $200 \mathrm{rpm}$ in $10 \mathrm{ml}$ MNGE medium containing $2 \%$ glucose, $0.2 \%$ potassium glutamate, $100 \mathrm{mM}$ potassium phosphate buffer (pH 7), $3.4 \mathrm{mM}$ trisodiumcitrate, $3 \mathrm{mM} \mathrm{MgSO}_{4}, 42 \mu \mathrm{M}$ ferric ammonium citrate, $0.24 \mathrm{mM}$ L-tryptophan and $0.1 \%$ casein hydrolysate. During the transition from exponential to stationary phase, the culture was diluted with another $10 \mathrm{ml}$ of MNGE medium (without casein hydrolysate) and incubated for $1 \mathrm{~h}$ at $37^{\circ} \mathrm{C}$ with shaking. In case of strain GP3187, $0.5 \%$ xylose was added to both media. Afterwards, $250 \mathrm{ng}$ of chromosomal DNA was added to $400 \mu \mathrm{l}$ of cells and incubated for 30 minutes at $37^{\circ} \mathrm{C}$. One hundred microliter of Expression mix (2.5\% yeast extract, $2.5 \%$ casein hydrolysate, $1.22 \mathrm{mM}$ tryptophan) was added and cells were allowed to grow for $1 \mathrm{~h}$ at $37^{\circ} \mathrm{C}$, before spreading onto selective LB plates containing appropriate antibiotics. 
Transformation of strains harboring comK and coms expressed from the mannitol inducible promotor $\left(\mathrm{P}_{m t / A}\right)$ was performed based on (Rahmer et al., 2015). Briefly, an overnight culture was diluted in $5 \mathrm{ml}$ LB to an initial $\mathrm{OD}_{600}$ of 0.1 and incubated at $37^{\circ} \mathrm{C}$ at $200 \mathrm{rpm}$. After 90 minutes incubation, $5 \mathrm{ml}$ of fresh LB containing mannitol (1\%) and $\mathrm{MgCl}_{2}(5 \mathrm{mM})$ were added and the bacterial culture was incubated for an additional 90 minutes. The cells were then pelleted by centrifugation for 10 minutes at 2,000 $\mathrm{x}$ and the pellet was re-suspended in the same amount of fresh LB medium, $1 \mathrm{ml}$ aliquots were distributed into $1.5 \mathrm{ml}$ reaction tubes and $250 \mathrm{ng}$ of chromosomal DNA was added to each of them. The cell suspension was incubated for $1 \mathrm{~h}$ at $37^{\circ} \mathrm{C}$ and transformants were selected on LB plates as described above.

\section{Plasmid construction}

All plasmids used in this study are listed in Table S4. Escherichia coli DH5 $\alpha$ (Sambrook et al., 1989) was used for plasmid constructions and transformation using standard techniques (Sambrook et al., 1989). To express the B. subtilis protein YtrF under the control of a xylose inducible promotor, we cloned the ytrF gene into the backbone of pGP888 via the Xbal and KpnI sites (Diethmaier et al., 2011).

\section{Biofilm assay}

To analyse biofilm formation, selected strains were grown in $\mathrm{LB}$ medium to an $\mathrm{OD}_{600}$ of about 0.5 to 0.8 and $10 \mu \mathrm{l}$ of the culture were spotted onto MSgg agar plates (Branda et al., 2001). Plates were incubated for 3 days at $30^{\circ} \mathrm{C}$.

\section{Fluorescence microscopy}

For fluorescence microscopy imaging, $B$. subtilis cultures were grown in $10 \mathrm{ml} \mathrm{MNGE}$ medium till the transition from exponential to stationary phase and then diluted with another 10 $\mathrm{ml}$ of MNGE medium as described for the transformation experiments (see above). $5 \mu$ l of cells were pipetted on microscope slides coated with a thin layer of $1 \%$ agarose and covered with a cover glass. Fluorescence images were obtained with the Axiolmager M2 fluorescence microscope, equipped with digital camera AxioCam MRm and AxioVision Rel 4.8 software for image processing and an EC Plan-NEOFLUAR 100X/1.3 objective (Carl Zeiss, Göttingen, Germany). Filter set 38 (BP 470/40, FT 495, BP 525/50; Carl Zeiss) was applied for GFP detection. Ratio of GFP expressing cells to the total number of cells was determined by manual examination from three 
independent randomly selected pictures originated from at least two independent growth replicates.

\section{Transmission electron microscopy}

To examine cell wall thickness of $B$. subtilis strains, cells were prepared for Transmission Electron Microscopy (TEM) as previously described (Rincón-Tomás et al., 2020). An overnight culture was inoculated to an $\mathrm{OD}_{600}$ of 0.05 in $30 \mathrm{ml}$ MNGE medium and grown to an $\mathrm{OD}_{600}$ of $0.6 \pm$ 0.1 at $37^{\circ} \mathrm{C}$ and $200 \mathrm{rpm}$. Cells were centrifuged for 10 minutes at $4,000 \mathrm{rpm}$ to obtain a $100 \mu \mathrm{l}$ cell pellet, which was then washed twice in phosphate-buffered saline (PBS, $127 \mathrm{mM} \mathrm{NaCl}, 2.7$ $\left.\mathrm{mM} \mathrm{KCl}, 10 \mathrm{mM} \mathrm{Na} \mathrm{HPO}_{4}, 1.8 \mathrm{mM} \mathrm{KH}_{2} \mathrm{PO}_{4}, \mathrm{pH} 7.4\right)$ and fixed overnight in $2.5 \%(\mathrm{w} / \mathrm{v})$ glutaraldehyde at $4^{\circ} \mathrm{C}$. Cells were then mixed with $1.5 \%$ (w/v, final concentration) molten BactoAgar (in PBS) and the resulting agar block was cut to pieces of $1 \mathrm{~mm}^{3}$. A dehydration series was performed ( $15 \%$ aqueous ethanol solution for 15 minutes, 30\%, 50\%, 70\% and $95 \%$ for 30 minutes and $100 \%$ for $2 \times 30$ minutes) at $0^{\circ} \mathrm{C}$, followed by an incubation step in $66 \%$ LR white resin mixture ( $v / v$, in ethanol) (Plano, Wletzlar, Germany) for 2 hours at room temperature and embedment in $100 \%$ LR-White solution overnight at $4^{\circ} \mathrm{C}$. One agar piece was transferred to a gelatin capsule filled with fresh LR-white resin, which was subsequently polymerized at $55^{\circ} \mathrm{C}$ for 24 hours. A milling tool (TM 60, Fa. Reichert \& Jung, Vienna, Austria) was used to shape the gelatin capsule into a truncated pyramid. An ultramicrotome (Reichert Utralcut E, Leica Microsystems, Wetzlar, Germany) and a diamond knife were used to obtain ultrathin sections $(80 \mathrm{~nm})$ of the samples. The resulting sections were mounted onto mesh specimen Grids (Plano, Wetzlar, Germany) and stained with $4 \%(\mathrm{w} / \mathrm{v})$ uranyl acetate solution $(\mathrm{pH} 7.0)$ for 10 minutes. Microscopy was performed in a Joel JEM 1011 transmission electron microscope (Joel Germany GmbH, Freising, Germany) at $80 \mathrm{kV}$. Images were taken at a magnification of 30,000 and recorded with a Gatan Orius SC1000 CCD camera (Gatan, Munich, Germany). For each replicate, 20 cells were photographed and cell wall thickness was measured at three different locations using ImageJ software (Rueden et al., 2017).

\section{Acknowledgements}

We wish to thank Julia Busse, Melin Güzel and Leon Daniau for the help with some experiments. We are grateful to Josef Altenbuchner, Jan Gundlach, Leendert Hamoen, Daniel Kearns, Daniel Reuss, Sarah Wilcken, and the Bacillus Genetic Sock Center for providing B. subtilis strains. We thank Dr. Michael Hoppert for providing access to the Transmission Electron Microscope. This research received funding from the Deutsche Forschungsgemeinschaft via SFB860. 


\section{Discussion}

\subsection{Suppressor mutant screen revealed initiation of bulk mRNA degradation as the pivotal function of RNase $Y$}

This thesis is focused on RNase $Y$ and the effect of the rny gene deletion on $B$. subtilis physiology. The rny gene was for a long time considered to be essential, but it could be later deleted from the genome in the study of Figaro et al. (2013). The authors of this study managed to delete the rny gene in genetic backgrounds of four different $B$. subtilis strains commonly used in the laboratories around the world and thus concluded that requirement for second-site suppressor mutations is rather unlikely. However, the rny mutant shows deformed cellular morphology, forms small and smooth colonies and has significantly decreased growth rate as compared to the wild type strain. Taken together, the rny deletion strain is far away from the optimal growth of $B$. subtilis and thus has a huge space for improvements of its properties through suppressor mutations. Indeed, although we were able to verify that it is possible to introduce an rny deletion into different strains of $B$. subtilis, we have observed that the rny mutant does lyse rather quickly followed by the appearance of suppressor colonies.

As already mentioned in the introduction, essential genes were defined as those whose deletion prevents growth under standard laboratory conditions; from that point of view the rny gene cannot be regarded as essential (Kobayashi et al., 2003; Commichau et al., 2013). However, dividing genes into only two groups of essential and non-essential genes is probably not the most appropriate. There are differences in the importance for cell growth even between the genes that would be traditionally marked as essential. This was recently evaluated in a study where the authors measured the time for which bacteria can continue to grow after disruption of a particular essential gene and thereby managed ordered the essential genes by their importance (Gallagher et al., 2020). In light of this study, essentiality should not be considered as yes or no question, but rather as a scale ranging from genes whose deletion does not cause any disadvantage to genes whose inactivation leads to immediate cell death. Since the rny deletion does not allow for robust growth and has to be compensated by second site suppressor mutations, we believe that the rny gene should be very close to the upper boundary on such a scale and therefore we decided to label this gene as a quasi-essential, also in accordance with the definition from Hutchison et al. (2016). This brings the question about the reason(s) for this quasiessentiality and about the main cellular functions of the enzyme.

There are several possible reasons for the pivotal role of RNase Y. Firstly, there might be a specific essential transcript that needs to be processed by the RNase. Indeed, it was previously 
shown that RNase $Y$ is involved in the maturation of two essential sRNAs, small cytoplasmic RNA (scRNA) coding for the ribonucleic components of the signal recognition particle (SRP), and $r n p B$ that encodes the ribozyme component of RNase P. Essentiality of scRNA lays in the role of SRP in co-translational trafficking of proteins to/across the cytoplasmic membrane and scRNA itself is responsible for translation arrest during this process by interaction with 23S RNA (Beckert et al., 2015; Tsirigotaki et al., 2017). RNase $P$ is responsible for maturation of the $5^{\prime}$ end of tRNAs (Guerrier-Takada et al., 1983). Given that both RNAs are essential, it would be tempting to speculate that the essentiality of RNase $Y$ lays in the absence of their respective processing events. However, scRNA was shown to be functional even in its unprocessed form (Beckert et al., 2015) and RNase P processing has alternative, although less efficient pathways, that are RNase $Y$ independent (Gilet et al., 2015). Therefore, the possibility that absence of processing of those two functional RNA molecules is the reason for RNase Y quasi-essentiality seems to be rather unlikely.

Except the already known essential targets, another option is that absence of processing of some so far unidentified target of RNase $Y$ stands behind the detrimental phenotypes and quasi-essentiality of the rny gene. This was, by analogy, shown for RNase III, which is essential thanks to its cleavage event in a prophage encoded toxin-antitoxin system (Durand et al., 2012b), or for RNase Z, which is responsible for tRNA processing (Pellegrini et al., 2003). To identify such a specific target, we decided to use the force of natural genetic selection and thus analyzed several of the suppressor colonies, popping up on the plates after the lyses of the rny mutant strain. We took several different colonies evolved at different conditions, to be able to identify whether the selection uses general mechanism or is condition-specific. Analysis of suppressor mutants has previously helped to uncover interconnections of metabolic pathways, important protein residues as well as reasons for essentiality of the signaling molecule c-di-AMP (Gundlach et al., 2017; Tödter et al., 2017; Osaka et al., 2020) and we thus hoped this approach to give us a better insight into the most important functions of RNase $\mathrm{Y}$.

The suppressor mutant analysis identified single nucleotide polymorphisms in the genes greA, rpoE and $c s p D$, coding for transcription elongation factor (Kusuya et al., 2011), the RNA polymerase subunit $\delta$ (Juang and Helmann, 1994; Rabatinová et al., 2013) and an RNA chaperone (Graumann et al., 1997), in dependence of isolation conditons. These three genes does not seem to play a crucial role for $B$. subtilis and thus it is unlikely, that they would be directly responsible for the rny mutant quassi-essentiality. On the other hand, they share a common function related to transcription, a process which is on the other side of RNA life span than the degradation initiated by RNase Y. Next to those mutations, we observed an interesting phenomenon present in all of the suppressors. That was a duplication of the $60 \mathrm{~kb}$ long fragment located between the ribosomal operons $r r n W$ and $r r n l$. Similar duplications of a larger genomic regions encompassed 
by ribosomal operons were noticed already 40 year ago for the gram-positive organisms $E$. coli and Salmonella typhimurium, since the highly similar rRNA coding operons are ideal platform for homologous recombination events (Hill et al., 1977; Anderson and Roth, 1981). Already back then in the study on S. typhimurium the authors remarked that the duplicated region contains all genes coding for the core subunits of the RNA polymerase and suggested that their duplication might have a major influence on the cellular physiology (Anderson and Roth, 1981). The situation seems to be similar in the gram-positive Bacillus subtilis, where the three genes for core subunits of the RNA polymerase are also present within this duplicated region we observed here.

With such a knowledge in mind and in relation to the point mutation found in transcription related genes, we assumed that the simultaneous duplication of the genes for the RNA polymerase core might be responsible for the observed suppression of the rny gene deletion. We were able to confirm this hypothesis with our next experiment (see Fig. 7), in which we deleted the rny gene in such a genetic background, where the three core polymerase genes are no longer present at the same genetic locus in between the $r r n W$ and $r r n /$ ribosomal operons and thus cannot be easily duplicated simultaneously. However, the new genetic composition of the RNA polymerase genes did not prevent the suppressor formation of the rny mutant completely. Even in this background the rny mutant formed suppressors extensively which allowed us to analyze this second class of suppressors. All of them carried single nucleotide polymorphisms directly in the genes coding for the RpoB and RpoC subunits of the RNA polymerase, leading to huge decrease in the transcriptional activity as we observed in subsequent in vitro transcription assays (see Fig. 9). The suppressing mechanism of the first class of suppressors containing RNA polymerase genes duplication in conjunction with transcription factors mutations seem to be less obvious, however according to the mathematic model presented in chapter 2 it is also assumed to decrease transcription rates significantly.Taken together the results from the suppressor screen suggested that there is not a single one specific transcript, whose degradation/processing would be the key function of RNase $Y$. Since RNase $Y$ is the enzyme responsible for initiation of the degradation of the majority of transcripts and the global mRNA half-lives are doubled in the rny depletion strain (Shahbabian et al., 2009; Lehnik-Habrink et al., 2011b), we concluded that it is likely the global role in degradation of bulk mRNA which stands behind the quasi-essentiality of RNase Y. The rny deletion likely leads to a never-ending accumulation of total mRNA (see Fig. 15), which results in high energy consumption and high degree of cellular stress. It would be logical if the suppressors would therefore either try to increase RNA degradation or decrease RNA synthesis, which seems to be the case for both classes of the isolated suppressors. 
A

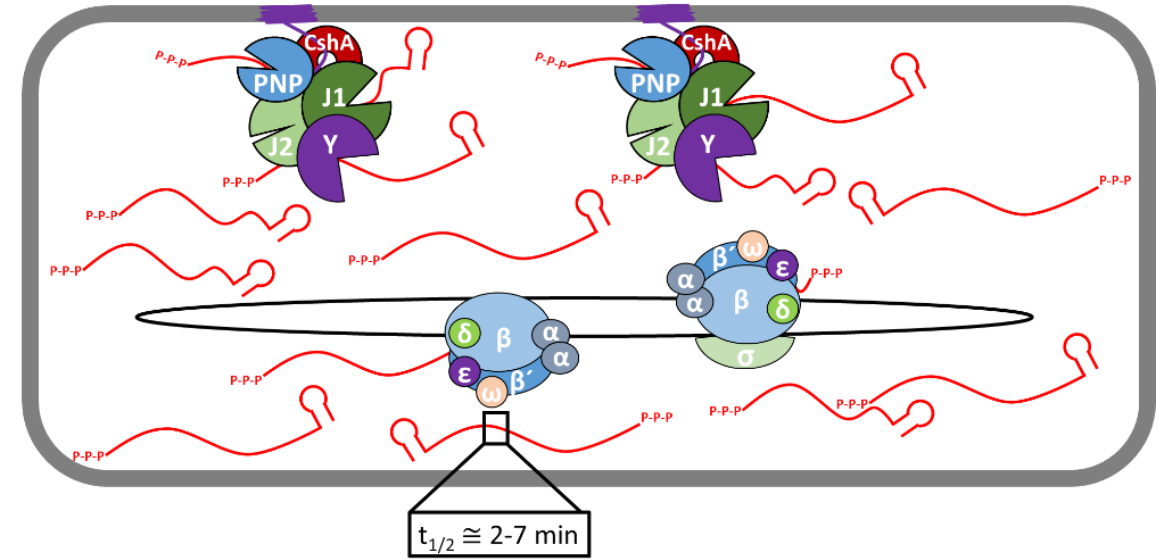

B

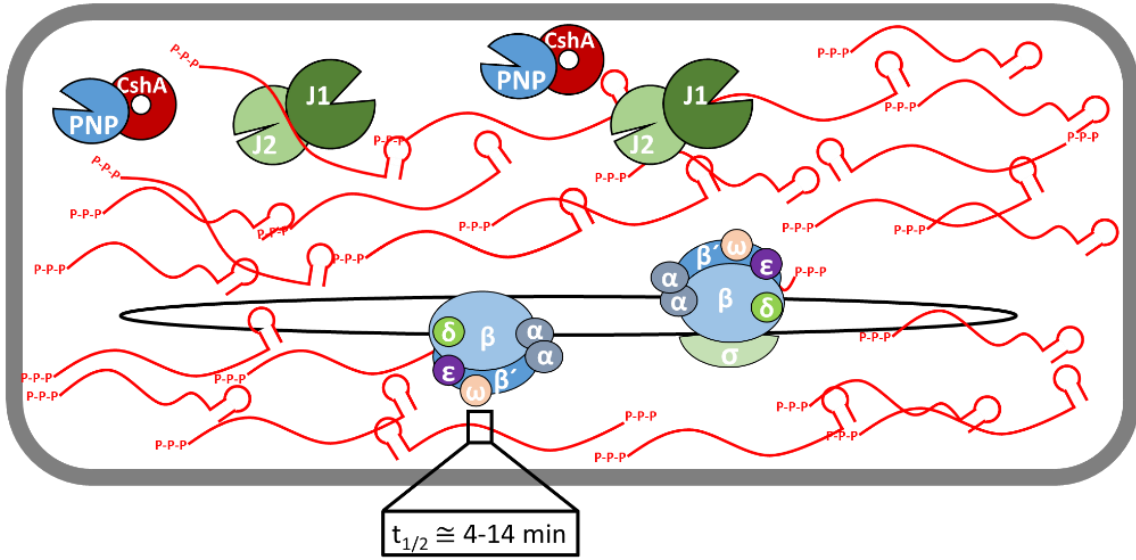

C

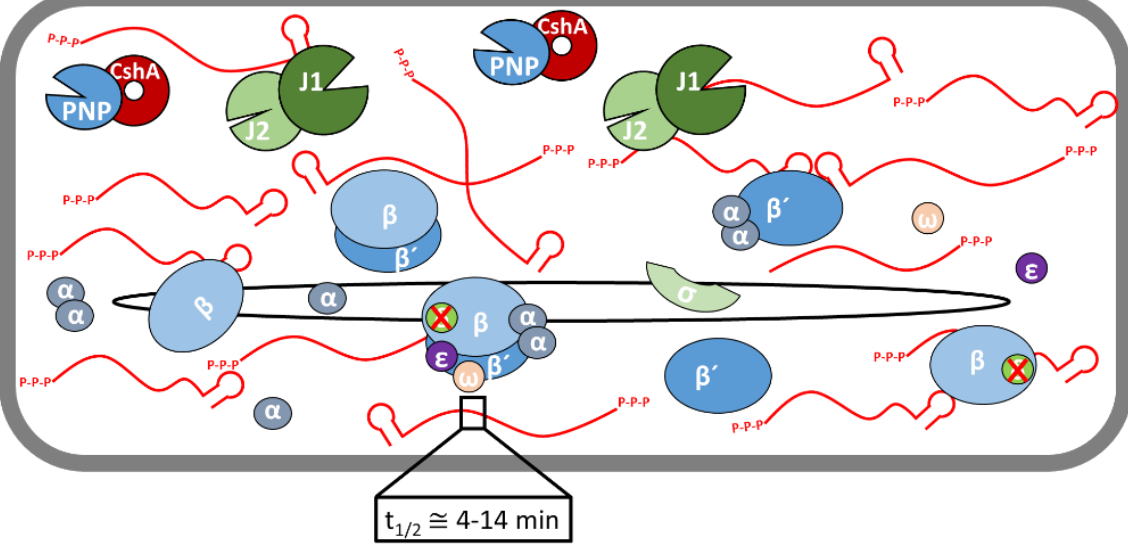

D

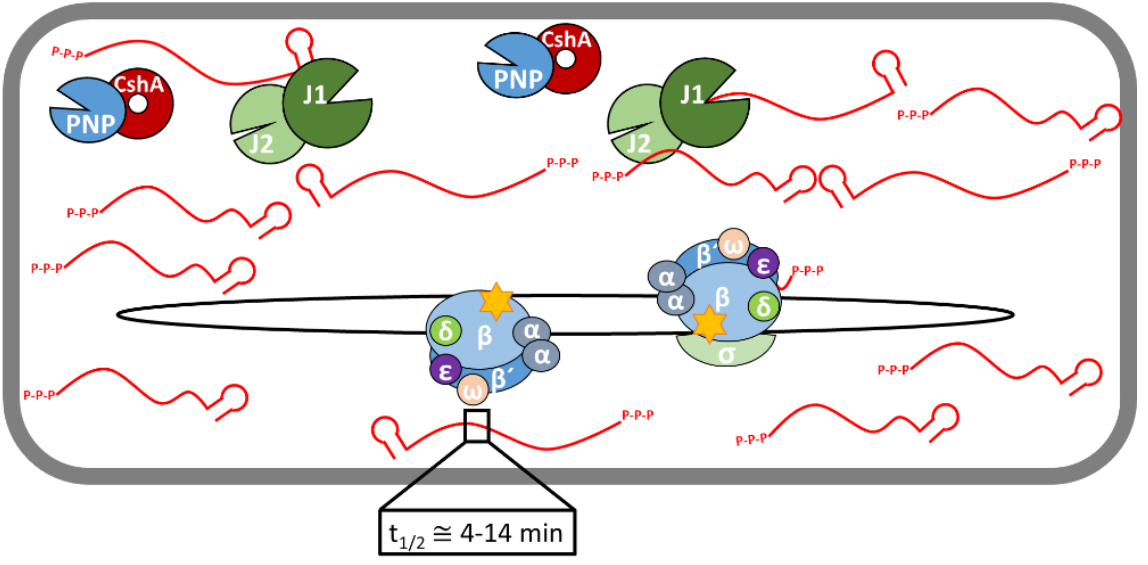


Figure 15: Model of RNA synthesis and degradation in wild type, the rny mutant and its suppressors In the case of wild type strain 168 (A), RNA molecules (red lines) are quickly transcribed from DNA template (black line) by the multi-subunit RNA polymerase composed of two subunits $\alpha$ (grey), subunit $\beta$ (light blue), $\beta^{\prime}$ (dark blue), $\omega$ (light orange), $\delta$ (green), $\varepsilon$ (violet) and during process of initiation also subunit $\sigma$ (light green). RNA is subsequently rapidly degraded as a result of initial cleavage by RNase $Y$ (purple), followed by exoribonucleolytic degradation by other degradosome-like network components PNPase (blue) and RNases $\mathrm{J} 1$ and J2 (light and dark green). This leads to balanced RNA equilibrium where majority of transcripts have half-lives of 2-7 minutes as observed by Hambraeus et al. (2003). In the rny mutant (B) RNA synthesis by the RNA polymerase proceeds as fast as in the wild type case, while the degradation is affected by the absence of RNase $Y$ and is achieved only to a limited extent due to the activity of other RNases, probably mainly RNase J1. This RNA degradation defect leads to about two-fold increased half-lives (approx. 4-14 minutes) as expected based on results from Shahbabian et al., (2009) and thereby to accumulation of total mRNA in the cell, which causes stress that the cells try to alleviate through formation of suppressors. In the suppressor mutants of the first class, e. g. strain GP2637 (C), RNA synthesis is affected by duplication of core RNA polymerase subunits $\left(\alpha, \beta, \beta^{\prime}\right)$. This leads to a significantly reduced likelihood that all subunits interact properly, and the number of fully functional RNA polymerase complexes is lower. Transcription is also further reduced by the presence of other mutations (indicated by red cross) in additional transcription factors, in the particular case of GP2637 the small RNA polymerase subunit $\delta$. Although the mRNA half-lives remain the same as in the case of the rny mutant, the total amount of mRNA molecules is reduced back towards the situation in the wild type strain due to the decreased transcription rates, thus the strain reaches a new stable equilibrium between the RNA synthesis and degradation. In the second class of suppressors, e. g. strain GP2912 (D), the slowdown in transcription is achieved directly through mutations (indicated by yellow asterisk) in the core subunits $\beta$ or $\beta^{\prime}$, which leads to reduced transcription rates. Similarly as in the case of first class suppressors, the mRNA half-lives remain the same as in the case of the rny mutant, but the number of mRNA molecules is lower and this way the strain finds a new stable equilibrium between the RNA synthesis and degradation.

For simplicity, the glycolytic enzymes which were proposed to be part of the B. subtilis degradosome-like network were omitted from this figure.

As judged from the absence of RNA degradation affecting suppressor mutations, the cells lacking RNase $\mathrm{Y}$ are apparently unable to increase the RNA degradation and thereby decrease the average half-lives back towards the situation in the wild type strain. Hence, the suppressor mutants had to use an alternative approach and limit RNA transcription. This way the average mRNA half-lives should stay increased as compared to the wild type strain, but thanks to the decreased transcription, the total RNA should not accumulate to such a huge extent, establishing a new stable equilibrium between the RNA synthesis and degradation and improving the energetic status of the cell. We had observed two alternative ways how the suppressor of the rny mutant achieve this. In the first class of suppressors, the core RNA polymerase genes are duplicated which, in conjunction with additional mutations in transcription related genes (greA, $\operatorname{csp} D, r p o E)$. This duplication leads to a decreased likelihood of proper RNA polymerase assembly and thereby decreased transcription. In the second class of suppressors harboring point mutations in the genes coding for core RNA polymerase subunits, we could clearly show that the transcriptions rates are significantly diminished.

Further experiments will be necessary to fully confirm this conclusion, however it might not be easy to find the proper experimental setup. The best approach would be to use either DNA microarrays or RNA-sequencing and analyze the transcriptomes of the wild-type, the rny mutant 
and its respective suppressor strains at various time intervals after rifampicin treatment. This rifampicin based approach coupled with DNA microarrays was previously used to determine mRNA half-lives in the wild type strain (Hambraeus et al., 2003) and would provide detailed information about both the speed of RNA decay as well as about the total mRNA abundance and abundance of individual transcripts. While such an experiment would certainly help to validate the conclusions drawn in this thesis and could provide further insights into the RNA metabolism in these strains, its performance in relevant triplicates would require dozens of different samples assessed by the transcriptomic approach, which seems to be excessive and unfeasible for routine laboratory work. As an alternative to this global approach, Northern blot assay or qRT-PCR analyses may be performed on selected genes. This was in past used for example to assess the roles of $3^{\prime}$-to-5' directed exoribonucleases in B. subtilis (Oussenko et al., 2005). However, such an approach always brings the risk that the results will be biased by the gene selection. It might be therefore at least interesting to see whether the bulk mRNA half-lives are indeed the same between the rny mutant and its suppressors. This could be potentially measured by pulse-labeling RNA with $\left[{ }^{3} \mathrm{H}\right]$ uridine as was done previously for instance to determine bulk mRNA decay rates in the pnpA mutant (Wang and Bechhofer, 1996).

Overall, we have concluded that it is the initiation of bulk mRNA degradation through the endoribonuclease cleavage that is the pivotal function of the RNase $Y$ and that is required to keep the RNA synthesis and degradation in a constant equilibrium. This finding is also in agreement with the evidence from E. coli, where similar conclusions were drawn about the essentiality of RNase E (Hammarlöf et al., 2015).

Interestingly, in any of the two suppressor screens we did not observe any mutations affecting the behavior of other RNases or components of the degradosome-like network (e.g. CshA). This suggests that RNase Y plays an important role, which cannot be easily substituted by any other enzyme encoded in the genome of $B$. subtilis. It would be, however, interesting to see, whether it is possible to replace the activity of RNase $Y$ with some RNase of other group, for instance RNase E of E. coli. In the opposite direction, it was already shown that RNase $Y$ can substitute the essential RNase E of $E$. coli, although the resulting strain was only able to grow on a minimal medium and does not reach wild type like growth rates (Tamura et al., 2017). It would be thus interesting to test whether this interchangeability is bidirectional.

Except $B$. subtilis, RNase $Y$ was extensively studied also in its two relatives $S$. aureus and S. pyogenes and is present in many other bacteria, including the gram-negative organisms Borrelia burgdorferi or Thermatoga maritima. Despite the fact that the homologs of RNase $\mathrm{Y}$ can be found in multiple bacterial species, their roles in cell physiology seem to be different. Interestingly, even the homologs of RNase $Y$ present in closely related organism of the Firmicutes phylum seem to 
play less important roles as judged from only a mild, if any, growth defects of the deletion mutants as compared to huge growth defects and phenotypic changes observed in case of the $B$. subtilis rny mutant. In fact, deletion of the rny genes in S. aureus and S. pyogenes is mainly connected to attenuated virulence rather than decreased growth rates in the laboratory conditions (Kang et al., 2010; Marincola et al., 2012; Khemici et al., 2015). Since those enzymes are highly similar (see Table 5 ) to the one of $B$. subtilis with $68.4 \%$ and $56.0 \%$ identity for the proteins of Staphylococcus aureus and Streptococcus pyogenes, respectively, it would be interesting to see whether those homologous proteins can substitute the one of $B$. subtilis. Such an experiment would allow us to discern whether the different requirements for RNase $Y$ presence in those organisms are caused by different enzymatic properties of the RNase $Y$ enzymes brought by the relatively small difference in the protein sequence or whether the RNA degradation is organized in a different manner in those species. This could be achieved for instance by increased role of another RNase on the global mRNA degradation as compared to $B$. subtilis. RNases $\mathrm{J} 1$ and $\mathrm{J} 2$ are promising candidates for that action. This is also supported by the fact that both single mutants lacking $S$. aureus RNases J and J2, respectively, show strong phenotypic defects (Linder et al., 2014), which is on the other hand not the case in B. subtilis.

Table 5: Comparison of RNase Y protein homology and rny mutant phenotypes among related species Organisms are indicated as follows: $B$. subtilis $=$ Bacillus subtilis, $L$. monocytogenes $=$ Listeria monocytogenes, S. aureus = Staphylococcus aureus, S. pyogenes = Streptococcus pyogenes, $\mathrm{C}$. difficile = Clostridioides difficile, $B$. burgdorferi = Borrelia burgdorferi

\begin{tabular}{|c|c|c|c|}
\hline Organism & Identity / Similarity ${ }^{\mathrm{a}}$ & Phenotype of the mutant & Source \\
\hline B. subtilis & $100.0 \% / 100.0 \%$ & $\begin{array}{c}\text { Major growth and } \\
\text { phenotypic defects; genomic } \\
\text { instability }\end{array}$ & Figaro et al., 2013 \\
\hline L. monocytogenes & $77.7 \% / 94.4 \%$ & ND & ND \\
\hline S. aureus & $68.4 \% / 90.4 \%$ & $\begin{array}{l}\text { Slight growth defect; } \\
\text { virulence attenuation }\end{array}$ & $\begin{array}{l}\text { Marincola et al., } 2012 \\
\text { Khemici et al., } 2015\end{array}$ \\
\hline C. difficile & $65.6 \% / 88.3 \%$ & Essential for growth & Dembek et al., 2015 \\
\hline S. pyogenes & $56.0 \% / 85.2 \%$ & $\begin{array}{l}\text { Slight growth defect only in } \\
\text { minimal media; } \\
\text { virulence attenuation }\end{array}$ & $\begin{array}{l}\text { Kang et al., } 2010 \\
\text { Chen et al., } 2013\end{array}$ \\
\hline B. burgdorferi & $45.6 \% / 78.3 \%$ & Essential for growth & Phelan et al., 2019 \\
\hline
\end{tabular}

${ }^{a}$ Identity and similarity values are relative to the $B$. subtilis protein; ND - not determined 
Another important variable to be addressed in future is the expression rate of RNase $Y$ necessary for stable growth. For its functional counterpart RNase E of $E$. coli, the presence of only $10-20 \%$ of wild type levels of the protein is sufficient to sustain normal growth and this reduction in RNase E quantity does not lead to major phenotypic effects (Jain et al., 2002). Such an information is unfortunately missing for RNase $Y$ of $B$. subtilis. Although transcriptomic studies with the inducible promoter based depletion of RNase $Y$ were performed, their experimental design does not allow us to calculate precisely the protein amount requirement for sustainable stable growth in wild type-like rates (Lehnik-Habrink et al., 2011b; Laalami et al., 2013).

It was previously suggested that mRNA turnover and generation time are correlated (Rustad et al., 2013). Although this seems to be the truth for the best studied model organisms among the domains of life (Bernstein et al., 2002; Hambraeus et al., 2003; Yang et al., 2003; Geisberg et al., 2014), there are exceptions breaking this concept. For instance the slowly growing cyanobacterium of the genus Prochlorococcus has a very short mRNA half-lives with average of 2,3 minutes, although it divides only once per day (Steglich et al., 2010). Furthermore, the generation times of $B$. subtilis, S. aureus and S. pyogenes are not that significantly different (Gera and Mclver, 2013; Missiakas and Schneewind, 2013) to explain the difference in phenotypes of the respective mutants. In addition, the rny mutant of $S$. pyogenes shows decreased growth rates only in minimal medium where the generation times are longer, however, if the hypothesis about generation time RNA stability correlation is correct, one would expect more severe phenotypes in rich media with shorter doubling times.

\subsection{Analysis of the rny suppressor mutants brings new insights into the regulation of the RNA polymerase}

Taken into an account the very strong difference in the activity of the RNA polymerase variants in in vitro transcription assays, which was 200 fold for the RpoC-R88H variant as compared to the wild type polymerase and not even quantifiable for the RpoB-G1054C variant, we can ask ourselves whether such a huge decrease in RNA polymerase activity really occurs in vivo. Although even just 2-fold increase in the mRNA half-lives is apparently enough to get the RNA synthesis/degradation rate significantly out of equilibrium (Shahbabian et al., 2009), the more than 200-fold drop in transcription activity still seems to be too excessive. Although further experimental evidence will be needed to fully address this question, the decrease in transcription rates is likely milder in vivo. In the gram-negative model organism E. coli it is well established that the levels of RpoB and RpoC subunits of the RNA polymerase are subject to an auto-regulation on multiple levels (Dennis et al., 1985; Meek and Hayward, 1986). Whether the RNA polymerase 
subunits are subject to a similar auto-regulation also in $B$. subtlis has never been addressed. Nevertheless, the presence of such auto-regulatory mechanism seems to be probable, not just as a rational explanation for the huge drop in in vitro transcriptional activity, but also judged from the increased protein quantity of the RNA polymerase RpoB subunit in the strains containing the RNA polymerase core mutations (both RpoB-G1054C and RpoC-R88H), which we have observed during Western-Blot experiments (data not shown).

In contrast to $E$. coli, where the $r p o B$ and $r p o C$ genes are part of a multicistronic operon together with ribosomal proteins, the $B$. subtilis $r p o B$ and $r p o C$ genes form just a bicistronic operon. This $r p o B C$ operon is, however, preceded by a more than $200 \mathrm{bp}$ long 5' UTR which could have an influence on the $r p o B C$ expression. Interestingly, a study published in the course of this thesis shown that RNase $Y$ cleaves within this UTR to create an alternative $5^{\prime}$ end of the rpoBC transcript (DeLoughery et al., 2018), giving rise to a possibility that RNase $Y$ is responsible for posttranscriptional regulation of $r p o B C$ expression in $B$. subtilis. Such an observation also sparked the attractive speculation that the absence of this cleavage by $R N a s e ~ Y$ is the reason for the formation of suppressor mutation affecting the RNA polymerase in response to the rny deletion. That would falsify our previous conclusion about the pivotal function of RNase $Y$ laying in the initiation of bulk mRNA degradation. However, such a possibility seems to be rather unlikely, since we did not observe any difference in the ß-galactosidase expression between the $\mathrm{P}_{r p o \beta}$-lacZ fusions containing or lacking the RNase $\mathrm{Y}$ cleavage site. Such a results suggests that the loss of the RNase $\mathrm{Y}$ cleavage site did not affect the expression of $r p o B C$ genes.

Although our aforementioned model clearly show that the probability of assembly of the whole RNAP complex is lower when core subunits are duplicated (see Fig. 11), there is one factor which was for calculation simplicity left out during the model construction, but might play a role in the suppression mechanism, and this is the presence of alternative sigma factors. The housekeeping factor $\sigma^{A}$ was the only sigma factor considered in the model, however, there are also 18 alternative sigma factors in $B$. subtilis. They are known to have lower affinity for the core than the housekeeping $\sigma^{A}$, which under normal circumstances contributes to the low expression of the genes under their control (Österberg et al., 2011). However, the alternative sigma factors may be favored in the situation with increased amount of uncomplete RNA polymerase complexes lacking some of the minor subunits. This was already shown on the example of $r p o Z$ mutant in other organisms, which showed increased proportion of transcription dependent on alternative sigma factors (Geertz et al., 2011; Gunnelius et al., 2014). Hence, it is possible that the effect of the core duplication might not only lead to decrease of the overall transcription, but also increase the proportion of transcripts from promoters controlled by the alternative sigma factors. 
This would together account for the positive effect on the physiology of the rny suppressors, since alternative sigma factors are mainly involved in transcription of stress related genes which might help to combat the phenotypes caused by the rny deletion.

Whether this is really the case and alternative sigma factors play a role in the suppression has to be assessed in future. On one hand, one might expect that cells that need increased transcription of genes dependent on alternative sigma factors would simply upregulate expression of the sigma factor for instance by promotor up mutations. However, on the other hand, a simultaneous decrease of $\sigma^{A}$ dependent transcription and increase in transcription from promoters controlled by multiple alternative sigma factors together might be most easily achieved by the duplication observed in our study, which is also supported by the finding that genomic amplifications are the easiest and most often occurring suppressing mutations in B. subtilis cells (Dormeyer et al., 2017; Reuß et al., 2019). One possible way to test the hypothesis about the alternative sigma factors involvement would be to introduce deletion of the rny gene into B. subtilis strain which was, on the other hand, proposed to have increased transcription activity from promoters dependent on the housekeeping sigma factor $\sigma^{A}$. That was shown for example for strains with rifampicin resistance variants of RpoB (Inaoka et al., 2004). If the hypothesis is correct, the rny deletion in such a background should lead to even more detrimental phenotype or obstacles in formation of suppressor mutations.

This thesis also brings strong support to the assumption that cold shock proteins, and especially $\mathrm{CspD}$, actually are transcription factors. This can be deduced from the finding of $\operatorname{cspD}$ affecting mutations in the one class of suppressors next to the mutations in genes for the known transcription factor greA and the RNA polymerase subunit rpoE. This assumption is further supported by the evidence that $\mathrm{CspB}$ and $\mathrm{CspD}$ are localized around the nucleoid in transcription dependent manner (Weber et al., 2001). Despite its name, cspD is expressed stably at variety of conditions (Nicolas et al., 2012) and its role in transcription would be in agreement with the role of the homologous cold-shock proteins in the gram-negative model organism $E$. coli, for which an anti-termination activity was proposed (Bae et al., 2000). Whether CspD and other so-called cold shock proteins in $B$. subtilis also act as anti-terminator proteins or whether their role in transcription is different has to be subject of further investigations.

Another interesting finding this thesis brings about the $\operatorname{cspD}$ gene is the fact, that the suppressors with inactivated $c s p D$ gene seem to be genetically stable (see Fig. S3), in contrast to the progenitor rny mutant as well as the other suppressors evolved under different selection scenarios that do provide a growth benefit, but do not lead to complete genetic stabilization. It is not completely clear whether this genetic stabilization upon $\operatorname{csp} D$ inactivation is specific to the rny mutant background or whether CspD plays some general role in the cellular ability to evolve 
mutations. Preliminary results obtained on that topic in our laboratory suggest, that this is rather rny specific, since double deletion strain of $\operatorname{cspD}$ and $\operatorname{cspB}$ is forming suppressor extensively (Faßhauer and Stülke, unpublished). However, what is the exact link between RNase Y, CspD and the genome stabilization remains unclear.

\subsection{Loss of RNase $Y$ leads to phenotypic effects independent of the total mRNA accumulation}

Whereas the total mRNA accumulation is likely the key problem the cells are facing upon the rny deletion, it does not explain all of the phenotypes observed in the rny mutant. There are probably additional reasons for some minor, less detrimental, phenotypes which could be connected to changed expression of specific genes. In order to get a better understanding of all the changes that occur upon the rny deletion we have used a transcriptomic approach. The wild type, the rny mutant and one of its suppressors were subjected to RNA-sequencing of transcripts present in the exponential phase of growth in rich medium. In agreement with previous studies (Lehnik-Habrink et al., 2011b; Durand et al., 2012a; Laalami et al., 2013), we could clearly see that the absence of RNase $Y$ leads to a global remodeling of mRNAs abundances, since expression of 1102 genes was at least two-fold different from the expression in the wild type strain, which means that $26 \%$ of all genes from the from the genome of $B$. subtilis are affected by the deletion. Besides, our screen undoubtedly did not identify all genes effected by the absence of RNase $Y$, since some genes with increased false discovery rates were excluded from the analysis and not all genes are expressed during the conditions chosen for this experiment, in fact only about $50 \%$ of all genes are transcribed during exponential growth in LB medium (Rasmussen et al., 2009). Therefore, we can conclude that loss of RNase $Y$ leads to global change of gene expression and influences abundance of majority of transcripts.

Since the rny mutant has severely impaired growth, we also cannot exclude the possibility, that differential expression of some genes which we observed is rather influenced by the growth-rate dependent regulation than directly by the rny deletion (Klumpp et al., 2009; Yubero and Poyatos, 2020).

Generally, we can divide the affected genes into two groups, those affected directly by the absence of RNase $Y$ and those where the differential is expression is caused indirectly. For a direct effect, one would expect that the loss of a specific cleavage leads to a stabilization of certain transcripts and destabilization of others. This is exactly the case of cggR-gapA operon. It was previously shown that RNase $Y$ cleaves between $\operatorname{cgg} R$ and gapA genes which leads to destabilization of cggR transcript (Commichau et al., 2009). Indeed, and also in agreement with 
previous transcriptomic studies, cggR had more than 7-fold higher abundance in the rny mutant as compared to the wild type (Lehnik-Habrink et al., 2011b; DeLoughery et al., 2018). Similarly, also expression of the $\mathrm{rpsO}$ transcript is destabilized by RNase $\mathrm{Y}$ cleavage and thus its abundance should be increased in the rny mutant, which was indeed the case in our study (Yao and Bechhofer, 2010).

However, for many genes, the expression may be affected indirectly, for instance as a consequence of differential expression of their regulators. This seems to be exactly the case if we consider the regulation by alternative sigma factors, where the $\sigma^{\mathrm{D}}$ dependent genes are mostly downregulated, likely in response to downregulation of $\operatorname{sig} D$ gene itself, while the $\sigma^{B}$ dependent genes are mainly upregulated, again probably due to $\operatorname{sig} B$ gene upregulation. These changes in the regulation of expression of alternative sigma factors may also not be a direct effect related to loss of RNase $\mathrm{Y}$ cleavage, but rather can be triggered by the overall stress that $r n y$ deletion exerts (Figaro et al., 2013), since especially transcription of $\sigma^{B}$ dependent genes is known to be part of the general stress response (Price et al., 2001).

An interesting example of the sigma factor dependency is the case of the $y v y C$ operon. This is an operon preceded by $\sigma^{\mathrm{D}}$ dependent promoter composed of 5 genes related to flagellar assembly $y v y C$, fliD, fliS, fliT, smiA and $h p f$ gene, coding for ribosome dimerization protein (Nicolas et al., 2012; Akanuma et al., 2016). All the first five genes of the operon are downregulated in response to $\operatorname{sig} D$ downregulation, however, the last gene, $h p f$, is not. This is likely the case because, except being part of the whole $\sigma^{D}$ dependent transcription unit, $h p f$ is also transcribed from two other promoters, dependent on $\sigma^{\mathrm{B}}$ and $\sigma^{\mathrm{H}}$, respectively (Drzewiecki et al., 1998).

In conjunction with our initial task addressed mainly in the suppressor mutant screen, we also tried to identify transcripts whose differential expression in the rny mutant would return to the wild type levels in the suppressing strain to alleviate the growth defects of the rny mutant. To that end we also analyzed the transcriptome of the suppressor strain GP2518. This strain had also a much-altered gene expression as compared both to the wild type (1168 differentially expressed genes), but also to the rny mutant. There are more than 150 transcripts that actually indeed returned towards the wild type levels in the suppressor. Given how large this group is, it is unlikely that the return of a single transcript level would be the key for the suppression observed. Already previous studies of transcriptomic effects of RNase $Y$ depletion did not manage to identify specific targets standing behind the crucial role of RNase Y for B. subtilis physiology (Laalami et al., 2013), supporting our previous conclusion that the role in regulation of global mRNA homeostasis is the main task of RNase $\mathrm{Y}$.

Previously, the only available transcriptomic data about the influence of RNase $\mathrm{Y}$ were obtained from depletion strains. Nevertheless, in parallel to this work, DeLoughery et al. (2018) 
published RNA-sequencing data of the rny deletion mutant and we thus wished to see, to what extent our data correlate. Our studies agreed in most cases for which data in sufficient quality were available in both studies, however not in all of them. For 54 genes (out of 1102 differentially expressed in our study) we observed an opposite effect in the two studies as compared to the respective wild-type levels. This discrepancy can by caused by the differences in experimental setups, since we have harvested the cells in higher $\mathrm{OD}_{600}$ than DeLoughery et al. and wild-wild type strain NCIB3610 was used in the other study and not laboratory wild type 168 as in our case. Interestingly, however, 38 out of those 54 genes, for which the expression data between our studies did not match, were also identified in our screen as genes whose expression returned towards the wild-type levels in the suppressor strain. It is therefore tempting to speculate that the rny mutant used by DeLoughery at el. had already acquired second site suppressor mutation(s) in the course of their experiment. This would not be so surprising given the incredible speed rny mutant forms suppressors and especially fixes the ctsR-pdaB duplication (see Fig. 10).

This is another noteworthy observation of this thesis. We have observed that deletion of the rny gene in the background of wild type 168 always leads to the maintenance of the ctsR$p d a B$ duplication, which is, however, naturally present also in a small part of the wild type population. It was already previously shown in gram-positive bacteria that stochastic duplications of chromosomal segments occur with a frequency ranging from $10^{-6}$ to $10^{-2}$ per cell per generation. Hence a standard population cultivated in the laboratory always contain cells harboring some genomic duplication and it is just a matter of probability, whether such a duplication brings any advantage to the cells and thus becomes dominant in the majority of the population (Pettersson et al., 2009; Tomanek et al., 2020). It was proposed by Romero and Palacios that such gene amplifications should not be considered as mutations, but rather as a dynamic state of the genome related to its fast adaptation preparedness for changing environmental conditions (Romero and Palacios, 1997). Apparently, these findings are valid also for the gram-positive $B$. subtilis. In fact, it took only 48 hours of growth inoculated with single colony for this ctsR-pdaB duplication to be maintained by the majority of the population, supporting the previous findings that duplications are a significantly faster mode of adaptation than other genome modifications, such as promoters up mutations, for example (Dormeyer et al., 2017; Reuß et al., 2019; Tomanek et al., 2020).

In correlation with the sigma factor dependency, as already suggested, we have noticed interesting patterns that might explain some of the observed phenotypes of the rny mutant. For instance, the downregulation of the sigD gene might explain the long chain phenotype, since it was shown that $\sigma^{D}$ OFF cells grow in long chains of sessile cells (Kearns and Losick, 2005). Under the $\sigma^{\mathrm{D}}$ control are also genes coding for five peptidoglycan autolysins (lytA, lytB, lytC, lytD and lytF) 
that play a major role in cell separation and motility (Chen et al., 2009). Since they were indeed all significantly downregulated in our transcriptomic analysis, it is tempting to speculate that their downregulation is responsible for the disordered peptidoglycan as observed in the rny mutant (Figaro et al., 2013). In an attempt to confirm such a hypothesis, it might be interesting to see, whether an artificial overexpression of either the autolysin genes, or of the sigD gene, would revert the phenotype of disordered peptidoglycan and growth in chains and possibly indirectly also affect other phenotypes observed in the rny mutant.

One such a phenotype which complicated the work in the laboratory and slowed down the progress of this project is the loss of genetic competence. To possibly speed up the progress, we decided to take a closer look at this phenomenon in the second part of this thesis. Initially we hypothesized that the loss of competence in the rny mutant strain may be a consequence of decreased expression of comK, the competence master regulator (van Sinderen et al., 1995). This was supported also by the transcriptomic data obtained in previous studies as well as in this thesis (Lehnik-Habrink et al., 2011b; Laalami et al., 2013). Furthermore RNase $Y$ is employed in maturation of sRNA called $r n a C$, which is responsible for maintaining levels of AbrB, transcriptional repressor of comK (Mars et al., 2015; DeLoughery et al., 2018). On top of that, the mecA transcript which encodes a protein responsible for ComK proteolytic degradation was shown to be more abundant in the rny mutant (DeLoughery et al., 2018). All these results together therefore suggested that the dysregulation of ComK levels through the aforementioned mechanisms could be behind the loss of competence in the rny mutant.

To test this possibility, we constructed a strain with overexpression of the comK and comS genes, comS encodes small adaptor protein which sequesters MecA-ClpCP complex and thereby prevents ComK degradation (Turgay et al., 1998; Prepiak and Dubnau, 2007), and introduced the rny deletion into such a background. If the competence deficiency of the rny mutant was really caused by the decreased expression of comK, transformation rates should be restored in this new background. However, this was not the case and the rny mutant did not give rise to a single transformant colony even upon comKS overexpression.

Having such a screening system in hand, we then decided to test some other genes, whose deletion also lead to the loss of genetic competence. This way we could show that nanoRNase $\mathrm{A}$ encoded by the gene $n r n A$ is involved in the regulation of competence master regulator Comk by so far undiscovered mechanism, or exclude the role of transcription factor GreA in the ComK regulation (van Sinderen et al., 1995; Mechold et al., 2007; Kusuya et al., 2011). These experiments also aroused our interested in the previously poorly characterized $A B C$ transporter YtrBCDEF (Yoshida et al., 2000; Salzberg et al., 2011). Expression of this transporter is controlled by the transcription repressor YtrA, whose deletion then leads to a loss of competence. 
It was previously shown that this repression is in the wild type strain relieved only upon very specific conditions related to cell wall attacking antibiotics (Salzberg et al., 2011; Wenzel et al., 2012).

Based on this we built and later confirmed the hypothesis that the expression of the YtrBCDEF transporter interferes with cell wall homeostasis and leads to increased cell wall thickness (see Fig. 14). We also suggested that such an interference with the cell wall properties can lead not only to a loss of genetic competence, but affect biofilm formation and sporulation (Koo et al., 2017) This data can in return shed light also on the reasons for the competence deficiency in the strain lacking RNase $Y$, since also this strain shows thicker and top of that highly disorganized peptidoglycan layer (Figaro et al., 2013), likely as a result of downregulated expression of autolysins as suggested above. By analogy to the situation in the ytrA mutant, it is very much possible that the DNA binding proteins simply does not reach out of the peptidoglycan layer to get in contact with the DNA molecule and that this steric hindrance is the main reason for the impossibility to transform the rny mutant. Another possibility, which is also connected to the function of autolysins, is that the chain growth prevents DNA binding, since DNA was shown to be bound to cell poles during the process of transformation and those are not free in the chaingrowing cells of the rny mutant (Hahn et al., 2005; Kidane and Graumann, 2005).

Taken together, this thesis brings an evidence about a highly dynamic system constantly looking for an optimal equilibrium between the cellular processes of RNA synthesis and degradation, which is severely affected in the absence of RNase $\mathrm{Y}$. In addition to the general role in global mRNA degradation, loss of RNase $\mathrm{Y}$ is also shown to effect directly or indirectly the expression of the majority of transcripts and some of them are suggested to provide explanation to some of the phenotypes connected with the deletion of the rny gene. 


\section{$5 \quad$ References}

Aguilar, C., Vlamakis, H., Guzman, A., Losick, R. and Kolter, R. (2010) KinD is a checkpoint protein linking spore formation to extracellular-matrix production in Bacillus subtilis biofilms. MBio 1: e00035-10.

Aguirre, A. A., Vicente, A. M., Hardwick, S. W., Alvelos, D. M., Mazzon, R. R., Luisi, B. F. and Marques, M. V. (2017) Association of the cold shock DEAD-box RNA helicase RhIE to the RNA degradosome in Caulobacter crescentus. J. Bacteriol. 199: e00135-17.

Akanuma, G., Kazo, Y., Tagami, K., Hiraoka, H., Yano, K., Suzuki, S. et al. (2016) Ribosome dimerization is essential for the efficient regrowth of Bacillus subtilis. Microbiology 162: 448-458.

Altenbuchner, J. (2016) Editing of the Bacillus subtilis genome by the CRISPR-Cas9 system. Appl. Environ. Microbiol. 82: 5421-5427.

Anderson, P. and Roth, J. (1981) Spontaneous tandem genetic duplications in Salmonella typhimurium arise by unequal recombination between rRNA (rrn) cistrons. Proc. Natl. Acad. Sci. U. S. A. 78: 3113-3117.

Andersson, D. I. and Hughes, D. (2009) Gene amplification and adaptive evolution in bacteria. Annu. Rev. Genet. 43: 167-195.

Aravind, L. and Koonin, E. V. (1998) The HD domain defines a new superfamily of metaldependent phosphohydrolases. Trends Biochem. Sci. 23: 469-472.

Baba, T., Ara, T., Hasegawa, M., Takai, Y., Okumura, Y., Baba, M. et al. (2006) Construction of Escherichia coli K-12 in-frame, single-gene knockout mutants: The Keio collection. Mol. Syst. Biol. 2: 2006.0008

Bae, W., Xia, B., Inouye, M. and Severinov, K. (2000) Escherichia coli CspA-family RNA chaperones are transcription antiterminators. Proc. Natl. Acad. Sci. U. S. A. 97: 7784-7789.

Barbe, V., Cruveiller, S., Kunst, F., Lenoble, P., Meurice, G., Sekowska, A. et al. (2009) From a consortium sequence to a unified sequence: The Bacillus subtilis 168 reference genome a decade later. Microbiology 155: 1758-1775.

Bechhofer, D. H. and Deutscher, M. P. (2019) Bacterial ribonucleases and their roles in RNA metabolism. Crit. Rev. Biochem. Mol. Biol. 54: 242-300.

Beckering, C. L., Steil, L., Weber, M. H. W., Völker, U. and Marahiel, M. A. (2002) Genomewide transcriptional analysis of the cold shock response in Bacillus subtilis. J. Bacteriol. 184: 6395-6402. 
Beckert, B., Kedrov, A., Sohmen, D., Kempf, G., Wild, K., Sinning, I. et al. (2015) Translational arrest by a prokaryotic signal recognition particle is mediated by RNA interactions. Nat. Struct. Mol. Biol. 22: 767-773.

Berka, R. M., Hahn, J., Albano, M., Draskovic, I., Persuh, M., Cui, X. et al. (2002) Microarray analysis of the Bacillus subtilis K-state: Genome-wide expression changes dependent on ComK. Mol. Microbiol. 43: 1331-1345.

Bernstein, J. A., Khodursky, A. B., Lin, P. H., Lin-Chao, S. and Cohen, S. N. (2002) Global analysis of mRNA decay and abundance in Escherichia coli at single-gene resolution using two-color fluorescent DNA microarrays. Proc. Natl. Acad. Sci. U. S. A. 99: 9697-9702.

Bernstein, J. A., Lin, P. H., Cohen, S. N. and Lin-Chao, S. (2004) Global analysis of Escherichia coli RNA degradosome function using DNA microarrays. Proc. Natl. Acad. Sci. 101: 2758-2763.

Beveridge, T. J. and Murray, R. G. E. (1979) How thick is the Bacillus subtilis cell wall? Curr. Microbiol. 2: 1-4.

Bolger, A. M., Lohse, M. and Usadel, B. (2014) Trimmomatic: A flexible trimmer for Illumina sequence data. Bioinformatics 30: 2114-2120.

Boonstra, M., Schaffer, M., Sousa, J., Morawska, L., Holsappel, S., Hildebrandt, P. et al. (2020) Analyses of competent and non-competent subpopulations of Bacillus subtilis reveal $y h f W, y h x C$ and ncRNAs as novel players in competence. Environ. Microbiol. 22: 2312-2328.

Branda, S. S., González-Pastor, J. E., Ben-Yehuda, S., Losick, R. and Kolter, R. (2001) Fruiting body formation by Bacillus subtilis. Proc. Natl. Acad. Sci. U. S. A. 98: 11621-11626.

Briley, K., Dorsey-Oresto, A., Prepiak, P., Dias, M. J., Mann, J. M. and Dubnau, D. (2011) The secretion ATPase ComGA is required for the binding and transport of transforming DNA. Mol. Microbiol. 81: 818-830.

Britton, R. A., Wen, T., Schaefer, L., Pellegrini, O., Uicker, W. C., Mathy, N. et al. (2007) Maturation of the $5^{\prime}$ end of Bacillus subtilis 16S rRNA by the essential ribonuclease YkqC/RNase J1. Mol. Microbiol. 63: 127-138.

Broglia, L., Lécrivain, A. L., Renault, T. T., Hahnke, K., Ahmed-Begrich, R., Rhun, A. Le and Charpentier, E. (2020) An RNA-seq based comparative approach reveals the transcriptome-wide interplay between 3'-to-5' exoRNases and RNase Y. Nat. Commun. 11: 1587.

Bruce, H. A., Du, D., Matak-Vinkovic, D., Bandyra, K. J., Broadhurst, R. W., Martin, E. et al. (2018) Analysis of the natively unstructured RNA/protein-recognition core in the Escherichia coli 
RNA degradosome and its interactions with regulatory RNA/Hfq complexes. Nucleic Acids Res. 46: 387-402.

Bruscella, P., Shahbabian, K., Laalami, S. and Putzer, H. (2011) RNase $Y$ is responsible for uncoupling the expression of translation factor IF3 from that of the ribosomal proteins L35 and L20 in Bacillus subtilis. Mol. Microbiol. 81: 1526-1541.

Bucher, T., Oppenheimer-Shaanan, Y., Savidor, A., Bloom-Ackermann, Z. and Kolodkin-Gal, I. (2015) Disturbance of the bacterial cell wall specifically interferes with biofilm formation. Environ. Microbiol. Rep. 7: 990-1004.

Cahová, H., Winz, M. L., Höfer, K., Nübel, G. and Jäschke, A. (2015) NAD captureSeq indicates NAD as a bacterial cap for a subset of regulatory RNAs. Nature 519: 374-377.

Cao, M., Wang, T., Ye, R. and Helmann, J. D. (2002) Antibiotics that inhibit cell wall biosynthesis induce expression of the Bacillus subtilis $\sigma^{\mathrm{W}}$ and $\sigma^{\mathrm{M}}$ regulons. Mol. Microbiol. 45: 1267-1276.

Carabetta, V. J., Silhavy, T. J. and Cristea, I. M. (2010) The response regulator SprE (RssB) is required for maintaining poly $(\mathrm{A})$ polymerase $\mathrm{I}$-degradosome association during stationary phase. J. Bacteriol. 192: 3713-3721.

Cardenas, P. P., Carrasco, B., Sanchez, H., Deikus, G., Bechhofer, D. H. and Alonso, J. C. (2009) Bacillus subtilis polynucleotide phosphorylase $3^{\prime}$-to- $5^{\prime}$ DNase activity is involved in DNA repair. Nucleic Acids Res. 37: 4157-4169.

Cardenas, P. P., Carzaniga, T., Zangrossi, S., Briani, F., Garcia-Tirado, E., Dehò, G. and Alonso, J. C. (2011) Polynucleotide phosphorylase exonuclease and polymerase activities on single-stranded DNA ends are modulated by RecN, SsbA and RecA proteins. Nucleic Acids Res. 39: 9250-9261.

Carpousis, A. J., Houwe, G. Van, Ehretsmann, C. and Krisch, H. M. (1994) Copurification of E. coli RNAase E and PNPase: Evidence for a specific association between two enzymes important in RNA processing and degradation. Cell 76: 889-900.

Carpousis, A. J. (2007) The RNA degradosome of Escherichia coli: An mRNA-degrading machine assembled on RNase E. Annu. Rev. Microbiol. 61: 71-87.

Cascante-Estepa, N., Gunka, K. and Stülke, J. (2016) Localization of components of the RNAdegrading machine in Bacillus subtilis. Front. Microbiol. 7: 1492.

Chang, B. Y. and Doi, R. H. (1990) Overproduction, purification, and characterization of Bacillus subtilis RNA polymerase $\sigma^{A}$ factor. J. Bacteriol. 172: 3257-3263. 
Chen, R., Guttenplan, S. B., Blair, K. M. and Kearns, D. B. (2009) Role of the $\sigma^{\mathrm{D}}$-dependent autolysins in Bacillus subtilis population heterogeneity. J. Bacteriol. 191: 5775-5784.

Chen, Z., Itzek, A., Malke, H., Ferretti, J. J. and Kreth, J. (2013) Multiple roles of RNase Y in Streptococcus pyogenes mRNA processing and degradation. J. Bacteriol. 195: 2585-2594.

Cho, K. H. (2017) The structure and function of the gram-positive bacterial RNA degradosome. Front. Microbiol. 8: 154.

Chung, D. H., Min, Z., Wang, B. C. and Kushner, S. R. (2010) Single amino acid changes in the predicted RNase $\mathrm{H}$ domain of Escherichia coli RNase $\mathrm{G}$ lead to complementation of RNase $\mathrm{E}$ deletion mutants. RNA 16: 1371-1385.

Clarke, J. E., Kime, L., Romero A., D. and McDowall, K. J. (2014) Direct entry by RNase E is a major pathway for the degradation and processing of RNA in Escherichia coli. Nucleic Acids Res. 42: $11733-11751$.

Clerget, M., Jin, D. J. and Weisberg, R. A. (1995) A zinc-binding region in the $\beta^{\prime}$ subunit of RNA polymerase is involved in antitermination of early transcription of phage HKO22. J. Mol. Biol 248: 768-780.

Clouet-d'Orval, B., Batista, M., Bouvier, M., Quentin, Y., Fichant, G., Marchfelder, A. and Maier, L. K. (2018) Insights into RNA-processing pathways and associated RNA-degrading enzymes in Archaea. FEMS Microbiol. Rev. 42: 579-613.

Coburn, G. A., Miao, X., Briant, D. J. and Mackie, G. A. (1999) Reconstitution of a minimal RNA degradosome demonstrates functional coordination between a $3^{\prime}$ exonuclease and a DEAD-box RNA helicase. Genes Dev. 13: 2594-2603.

Commichau, F. M., Rothe, F. M., Herzberg, C., Wagner, E., Hellwig, D., Lehnik-Habrink, M. et al. (2009) Novel activities of glycolytic enzymes in Bacillus subtilis: interactions with essential proteins involved in mRNA processing. Mol. Cell. Proteomics 8: 1350-1360.

Commichau, F. M., Pietack, N. and Stülke, J. (2013) Essential genes in Bacillus subtilis: A reevaluation after ten years. Mol. Biosyst. 9: 1068-1075.

Corrigan, R. M., Campeotto, I., Jeganathan, T., Roelofs, K. G., Lee, V. T. and Gründling, A. (2013) Systematic identification of conserved bacterial c-di-AMP receptor proteins. Proc. Natl. Acad. Sci. U. S. A. 110: 9084-9089.

Coutts, G., Thomas, G., Blakey, D. and Merrick, M. (2002) Membrane sequestration of the signal transduction protein GInK by the ammonium transporter AmtB. EMBO J. 21: 536-545. 
Czyz, A., Mooney, R. A., laconi, A. and Landick, R. (2014) Mycobacterial RNA polymerase requires a U-tract at intrinsic terminators and is aided by NusG at suboptimal terminators. MBio 5: e0093114.

Daou-Chabo, R., Mathy, N., Bénard, L. and Condon, C. (2009) Ribosomes initiating translation of the $h$ bs mRNA protect it from 5'-to-3' exoribonucleolytic degradation by RNase J1. Mol. Microbiol. 71: $1538-1550$.

Davies, K. M., Dedman, A. J., Horck, S. van and Lewis, P. J. (2005) The NusA:RNA polymerase ratio is increased at sites of rRNA synthesis in Bacillus subtilis. Mol. Microbiol. 57: 366-379.

Deana, A. and Belasco, J. G. (2004) The function of RNase G in Escherichia coli is constrained by its amino and carboxyl termini. Mol. Microbiol. 51: 1205-1217.

Deana, A., Celesnik, H. and Belasco, J. G. (2008) The bacterial enzyme RppH triggers messenger RNA degradation by 5' pyrophosphate removal. Nature 451: 355-358.

Deikus, G., Condon, C. and Bechhofer, D. H. (2008) Role of Bacillus subtilis RNase J1 endonuclease and $5^{\prime}$-exonuclease activities in trp leader RNA turnover. J. Biol. Chem. 283: 1715817167.

Deikus, G. and Bechhofer, D. H. (2009) Bacillus subtilis trp leader RNA. RNase J1 endonuclease cleavage specificity and PNPase processing. J. Biol. Chem. 284: 26394-26401.

DeLoughery, A., Dengler, V., Chai, Y. and Losick, R. (2016) Biofilm formation by Bacillus subtilis requires an endoribonuclease-containing multisubunit complex that controls mRNA levels for the matrix gene repressor SinR. Mol. Microbiol. 99: 425-437.

DeLoughery, A., Lalanne, J. B., Losick, R. and Li, G. W. (2018) Maturation of polycistronic mRNAs by the enribonuclease RNase $\mathrm{Y}$ and its associated Y-complex in Bacillus subtilis. Proc. Natl. Acad. Sci. U. S. A. 115: E5585-E5594.

Delumeau, O., Lecointe, F., Muntel, J., Guillot, A., Guédon, E., Monnet, V. et al. (2011) The dynamic protein partnership of RNA polymerase in Bacillus subtilis. Proteomics 11: 2992-3001.

Dembek, M., Barquist, L., Boinett, C. J., Cain, A. K., Mayho, M., Lawley, T. D. et al. (2015) Highthroughput analysis of gene essentiality and sporulation in Clostridium difficile. MBio 6: e02383.

Dennis, P. P., Nene, V. and Glass, R. E. (1985) Autogenous posttranscriptional regulation of RNA Polymerase $ß$ and $ß^{\prime}$ subunit synthesis in Escherichia coli. J. Bacteriol. 161: 803-806.

Detsch, C. and Stülke, J. (2003) Ammonium utilization in Bacillus subtilis: Transport and regulatory functions of NrgA and NrgB. Microbiology 149: 3289-3297. 
Diethmaier, C., Pietack, N., Gunka, K., Wrede, C., Lehnik-Habrink, M., Herzberg, C. et al. (2011) A novel factor controlling bistability in Bacillus subtilis: The Ymdb protein affects flagellin expression and biofilm formation. J. Bacteriol. 193: 5997-6007.

Doherty, G. P., Fogg, M. J., Wilkinson, A. J. and Lewis, P. J. (2010) Small subunits of RNA polymerase: Localization, levels and implications for core enzyme composition. Microbiology 156: 3532-3543.

Dormeyer, M., Lübke, A. L., Müller, P., Lentes, S., Reuß, D. R., Thürmer, A. et al. (2017) Hierarchical mutational events compensate for glutamate auxotrophy of a Bacillus subtilis gltC mutant. Environ. Microbiol. Rep. 9: 279-289.

Draskovic, I. and Dubnau, D. (2005) Biogenesis of a putative channel protein, ComEC, required for DNA uptake: Membrane topology, oligomerization and formation of disulphide bonds. Mol. Microbiol. 55: 881-896.

Drzewiecki, K., Eymann, C., Mittenhuber, G. and Hecker, M. (1998) The yvyD gene of Bacillus subtilis is under dual control of $\sigma(B)$ and $\sigma(H)$. J. Bacteriol. 180: 6674-6680.

Durand, S., Gilet, L., Bessières, P., Nicolas, P. and Condon, C. (2012a) Three essential ribonucleases-RNase $\mathrm{Y}, \mathrm{J} 1$, and III-control the abundance of a majority of Bacillus subtilis mRNAs. PLoS Genet. 8: e1002520.

Durand, S., Gilet, L. and Condon, C. (2012b) The essential function of B. subtilis RNase III is to silence foreign toxin genes. PLoS Genet. 8: e1003181.

Durand, S., Tomasini, A., Braun, F., Condon, C. and Romby, P. (2015) sRNA and mRNA turnover in gram-positive bacteria. FEMS Microbiol. Rev. 39: 316-330.

Durand, S., Braun, F., Helfer, A. C., Romby, P. and Condon, C. (2017) sRNA-mediated activation of gene expression by inhibition of 5'-3' exonucleolytic mRNA degradation. Elife 6: e23602.

Durand, S. and Condon, C. (2018) RNases and helicases in Gram-positive bacteria. Microbiol. Spectr. 6: RWR-0003-2017.

Even, S., Pellegrini, O., Zig, L., Labas, V., Vinh, J., Bréchemmier-Baey, D. and Putzer, H. (2005) Ribonucleases J1 and J2: Two novel endoribonucleases in B. subtilis with functional homology to E.coli RNase E. Nucleic Acids Res. 33: 2141-2152.

Evguenieva-Hackenberg, E., Hou, L., Glaeser, S. and Klug, G. (2014) Structure and function of the archaeal exosome. Wiley Interdiscip. Rev. RNA 5: 623-635. 
Fang, M., Zeisberg, W.-M., Condon, C., Ogryzko, V., Danchin, A. and Mechold, U. (2009) Degradation of nanoRNA is performed by multiple redundant RNases in Bacillus subtilis. Nucleic Acids Res. 37: 5114-5125.

Farr, G. A., Oussenko, I. A. and Bechhofer, D. H. (1999) Protection against 3'-to-5' RNA decay in Bacillus subtilis. J. Bacteriol. 181: 7323-7330.

Figaro, S., Durand, S., Gilet, L., Cayet, N., Sachse, M. and Condon, C. (2013) Bacillus subtilis mutants with knockouts of the genes encoding ribonucleases RNase $Y$ and RNase J1 are viable, with major defects in cell morphology, sporulation, and competence. J. Bacteriol. 195: 23402348.

Flórez, L. A., Gunka, K., Polanía, R., Tholen, S. and Stülke, J. (2011) SPABBATS: A pathwaydiscovery method based on Boolean satisfiability that facilitates the characterization of suppressor mutants. BMC Syst. Biol. 5: 5.

Frindert, J., Zhang, Y., Nübel, G., Kahloon, M., Kolmar, L., Hotz-Wagenblatt, A. et al. (2018) Identification, biosynthesis, and decapping of NAD-Capped RNAs in B. subtilis. Cell Rep. 24: 18901901.

Frindert, J., Kahloon, M. A., Zhang, Y., Ahmed, Y. L., Sinning, I. and Jäschke, A. (2019) Yvcl from Bacillus subtilis has in vitro RNA pyrophosphohydrolase activity. J. Biol. Chem. 294: 19967-19977.

Gallagher, L. A., Bailey, J. and Manoil, C. (2020) Ranking essential bacterial processes by speed of mutant death. Proc. Natl. Acad. Sci. U. S. A. 202001507.

Gamba, P., Jonker, M. J. and Hamoen, L. W. (2015) A novel feedback loop that controls bimodal expression of genetic competence. PLoS Genet. 11: e1005047.

Gao, J., Lee, K., Zhao, M., Qiu, J., Zhan, X., Saxena, A. et al. (2006) Differential modulation of $E$. coli mRNA abundance by inhibitory proteins that alter the composition of the degradosome. Mol. Microbiol. 61: 394-406.

Geertz, M., Travers, A., Mehandziska, S., Sobetzko, P., Janga, S. C., Shimamoto, N. and Muskhelishvilia, G. (2011) Structural coupling between RNA polymerase composition and DNA supercoiling in coordinating transcription: A global role for the omega subunit? MBio 2: e0003411.

Geisberg, J. V., Moqtaderi, Z., Fan, X., Ozsolak, F. and Struhl, K. (2014) Global analysis of mRNA isoform half-lives reveals stabilizing and destabilizing elements in yeast. Cell 156: 812-824. 
Gera, K. and Mclver, K. S. (2013) Laboratory Growth and Maintenance of Streptococcus pyogenes (The Group A Streptococcus, GAS). Curr Protoc Microbiol. 30: 9D.2.1-9D.2.13.

Gilet, L., Dichiara, J. M., Figaro, S., Bechhofer, D. H. and Condon, C. (2015) Small stable RNA maturation and turnover in Bacillus subtilis. Mol. Microbiol. 95: 270-282.

González-Gutiérrez, J. A., Díaz-Jiménez, D. F., Vargas-Pérez, I., Guillén-Solís, G., Stülke, J. and Olmedo-Álvarez, G. (2018) The DEAD-box RNA helicases of Bacillus subtilis as a model to evaluate genetic compensation among duplicate genes. Front. Microbiol. 9: 2261.

Graumann, P., Wendrich, T. M., Weber, M. H. W., Schröder, K. and Marahiel, M. A. (1997) A family of cold shock proteins in Bacillus subtilis is essential for cellular growth and for efficient protein synthesis at optimal and low temperatures. Mol. Microbiol. 25: 741-756.

Grishin, N. V (2001) KH domain: one motif, two folds. Nucleic Acids Res. 29: 638-643.

Grunberg-Manago, M., Ortiz, P. J. and Ochoa, S. (1956) Enzymic synthesis of polynucleotides I. polynucleotide phosphorylase of Azotobacter vinelandii. Biochim. Biophys. Acta - Gen. Subj. 20: 269-285.

Guérout-Fleury, A. M., Shazand, K., Frandsen, N. and Stragier, P. (1995) Antibiotic-resistance cassettes for Bacillus subtilis. Gene 167: 335-336.

Guerrier-Takada, C., Gardiner, K., Marsh, T., Pace, N. and Altman, S. (1983) The RNA moiety of ribonuclease $\mathrm{P}$ is the catalytic subunit of the enzyme. Cell 35: 849-857.

Gundlach, J., Herzberg, C., Kaever, V., Gunka, K., Hoffmann, T., Weiß, M. et al. (2017) Control of potassium homeostasis is an essential function of the second messenger cyclic di-AMP in Bacillus subtilis. Sci. Signal. 10: eaal3011.

Gundlach, J., Krüger, L., Herzberg, C., Turdiev, A., Poehlein, A., Tascón, I. et al. (2019) Sustained sensing in potassium homeostasis: Cyclic di-AMP controls potassium uptake by KimA at the levels of expression and activity. J. Biol. Chem. 294: 9605-9614.

Gunnelius, L., Hakkila, K., Kurkela, J., Wada, H., Tyystjärvi, E. and Tyystjärvi, T. (2014) The omega subunit of the RNA polymerase core directs transcription efficiency in cyanobacteria. Nucleic Acids Res. 42: 4606-4614.

Hadjeras, L., Poljak, L., Bouvier, M., Morin-Ogier, Q., Canal, I., Cocaign-Bousquet, M. et al. (2019) Detachment of the RNA degradosome from the inner membrane of Escherichia coli results in a global slowdown of mRNA degradation, proteolysis of RNase $E$ and increased turnover of ribosome-free transcripts. Mol. Microbiol. 111: 1715-1731. 
Hahn, J., Roggiani, M. and Dubnau, D. (1995) The major role of SpoOA in genetic competence is to downregulate $a b r B$, an essential competence gene. J. Bacteriol. 177: 3601-3605.

Hahn, J., Maier, B., Haijema, B. J., Sheetz, M. and Dubnau, D. (2005) Transformation proteins and DNA uptake localize to the cell poles in Bacillus subtilis. Cell 122: 59-71.

Haijema, B.-J., Hahn, J., Haynes, J. and Dubnau, D. (2001) A ComGA-dependent checkpoint limits growth during the escape from competence. Mol. Microbiol. 40: 52-64.

Hambraeus, G., Wachenfeldt, C. von and Hederstedt, L. (2003) Genome-wide survey of mRNA half-lives in Bacillus subtilis identifies extremely stable mRNAs. Mol. Genet. Genomics 269: 706714.

Hammarlöf, D. L., Bergman, J. M., Garmendia, E. and Hughes, D. (2015) Turnover of mRNAs is one of the essential functions of RNase E. Mol. Microbiol. 98: 34-45.

Hamoen, L. W., Werkhoven, A. F. Van, Venema, G. and Dubnau, D. (2000) The pleiotropic response regulator DegU functions as a priming protein in competence development in Bacillus subtilis. Proc. Natl. Acad. Sci. U. S. A. 97: 9246-9251.

Hamoen, L. W., Smits, W. K., Jong, A. de, Holsappel, S. and Kuipers, O. P. (2002) Improving the predictive value of the competence transcription factor (ComK) binding site in Bacillus subtilis using a genomic approach. Nucleic Acids Res. 30: 5517-5528.

Hamoen, L. W., Kausche, D., Marahiel, M. A., Sinderen, D. van, Venema, G. and Serror, P. (2003a) The Bacillus subtilis transition state regulator AbrB binds to the -35 promoter region of comK. FEMS Microbiol. Lett. 218: 299-304.

Hamoen, L. W., Venema, G. and Kuipers, O. P. (2003b) Controlling competence in Bacillus subtilis: shared use of regulators. Microbiology 149: 9-17.

Hamouche, L., Billaudeau, C., Rocca, A., Chastanet, A., Ngo, S., Laalami, S. and Putzer, H. (2020) Dynamic membrane localization of RNase Y in Bacillus subtilis. MBio 11: e03337-19.

Hardwick, S. W., Chan, V. S. Y., Broadhurst, R. W. and Luisi, B. F. (2011) An RNA degradosome assembly in Caulobacter crescentus. Nucleic Acids Res. 39: 1449-1459.

Hill, C. W., Grafstrom, R. H., Harnish, B. W. and Hillman, B. S. (1977) Tandem duplications resulting from recombination between ribosomal RNA genes in Escherichia coli. J. Mol. Biol. 116: 407-428.

Himabindu, P. and Anupama, K. (2017) Decreased expression of stable RNA can alleviate the lethality associated with RNase E deficiency in Escherichia coli. J. Bacteriol. 199: e00724-16. 
Hoa, T. T., Tortosa, P., Albano, M. and Dubnau, D. (2002) Rok (YkuW) regulates genetic competence in Bacillus subtilis by directly repressing comK. Mol. Microbiol. 43: 15-26.

Huen, J., Lin, C. L., Golzarroshan, B., Yi, W. L., Yang, W. Z. and Yuan, H. S. (2017) Structural insights into a unique dimeric DEAD-box helicase CshA that promotes RNA decay. Structure 25: 469-481.

Hunger, K., Beckering, C. L., Wiegeshoff, F., Graumann, P. L. and Marahiel, M. A. (2006) Coldinduced putative DEAD-box RNA helicases CshA and CshB are essential for cold adaptation and interact with cold shock protein B in Bacillus subtilis. J. Bacteriol. 188: 240-248.

Hunt, A., Rawlins, J. P., Thomaides, H. B. and Errington, J. (2006) Functional analysis of 11 putative essential genes in Bacillus subtilis. Microbiology 152: 2895-2907.

Hutchison, C. A., Chuang, R.-Y., Noskov, V. N., Assad-Garcia, N., Deerinck, T. J., Ellisman, M. H. et al. (2016) Design and synthesis of a minimal bacterial genome. Science 351: aad6253.

Hutter, B., Fischer, C., Jacobi, A., Schaab, C. and Loferer, H. (2004) Panel of Bacillus subtilis reporter strains indicative of various modes of action. Antimicrob. Agents Chemother. 48: 25882594.

Inaoka, T., Takahashi, K., Yada, H., Yoshida, M. and Ochi, K. (2004) RNA Polymerase mutation activates the production of a dormant antibiotic 3,3'-neotrehalosadiamine via an autoinduction mechanism in Bacillus subtilis. J. Biol. Chem. 279: 3885-3892.

Ingham, C. J., Dennis, J. and Furneaux, P. A. (1999) Autogenous regulation of transcription termination factor Rho and the requirement for Nus factors in Bacillus subtilis. Mol. Microbiol. 31: 651-663.

Jain, C., Deana, A. and Belasco, J. G. (2002) Consequences of RNase E scarcity in Escherichia coli. Mol. Microbiol. 43: 1053-1064.

Juang, Y. L. and Helmann, J. D. (1994) The $\delta$ subunit of Bacillus subtilis RNA polymerase: An allosteric effector of the initiation and core-recycling phases of transcription. J. Mol. Biol. 239: 114.

Kampf, J., Gerwig, J., Kruse, K., Cleverley, R., Dormeyer, M., Grünberger, A. et al. (2018) Selective pressure for biofilm formation in Bacillus subtilis: Differential effect of mutations in the master regulator SinR on bistability. MBio 9: e01464-18. 
Kang, S. O., Caparon, M. G. and Cho, K. H. (2010) Virulence gene regulation by CvfA, a putative RNase: the CvfA-enolase complex in Streptococcus pyogenes links nutritional stress, growth-phase control, and virulence gene expression. Infect. Immun. 78: 2754-2767.

Kearns, D. B. and Losick, R. (2005) Cell population heterogeneity during growth of Bacillus subtilis. Genes Dev. 19: 3083-3094.

Kearse, M., Moir, R., Wilson, A., Stones-Havas, S., Cheung, M., Sturrock, S. et al. (2012) Geneious Basic: An integrated and extendable desktop software platform for the organization and analysis of sequence data. Bioinformatics 28: 1647-1649.

Keller, A. N., Yang, X., Wiedermannová, J., Delumeau, O., Krásný, L. and Lewis, P. J. (2014) $\varepsilon$, a new subunit of RNA polymerase found in gram-positive bacteria. J. Bacteriol. 196: 3622-3632.

Khemici, V., Toesca, I., Poljak, L., Vanzo, N. F. and Carpousis, A. J. (2004) The RNase E of Escherichia coli has at least two binding sites for DEAD-box RNA helicases: functional replacement of RhIB by RhIE. Mol. Microbiol. 54: 1422-1430.

Khemici, V., Poljak, L., Luisi, B. F. and Carpousis, A. J. (2008) The RNase E of Escherichia coli is a membrane-binding protein. Mol. Microbiol. 70: 799-813.

Khemici, V., Prados, J., Linder, P., Redder, P. and Linder, P. (2015) Decay-initiating endoribonucleolytic cleavage by RNase $\mathrm{Y}$ is kept under tight control via sequence preference and sub-cellular localisation. PLOS Genet. 11: e1005577.

Kidane, D. and Graumann, P. L. (2005) Intracellular protein and DNA dynamics in competent Bacillus subtilis cells. Cell 122: 73-84.

Kido, M., Yamanaka, K., Mitani, T., Niki, H., Ogura, T. and Hiraga, S. (1996) RNase E polypeptides lacking a carboxyl-terminal half suppress a mukB mutation in Escherichia coli. J. Bacteriol. 178: 3917-3925.

Kim, S. K., Lormand, J. D., Weiss, C. A., Eger, K. A., Turdiev, H., Turdiev, A. et al. (2019) A dedicated diribonucleotidase resolves a key bottleneck for the terminal step of RNA degradation. Elife 8: e46313.

Klumpp, S., Zhang, Z. and Hwa, T. (2009) Growth-rate dependent global effects on gene expression in bacteria. Cell 139: 1366-1375.

Kobayashi, K., Ehrlich, S. D., Albertini, A., Amati, G., Andersen, K. K., Arnaud, M. et al. (2003) Essential Bacillus subtilis genes. Proc. Natl. Acad. Sci. U. S. A. 100: 4678-4683. 
Konkol, M. A., Blair, K. M. and Kearns, D. B. (2013) Plasmid-encoded Coml inhibits competence in the ancestral 3610 strain of Bacillus subtilis. J. Bacteriol. 195: 4085-4093.

Koo, B. M., Kritikos, G., Farelli, J. D., Todor, H., Tong, K., Kimsey, H. et al. (2017) Construction and analysis of two genome-scale deletion libraries for Bacillus subtilis. Cell Syst. 4: 291-305.

Kouba, T., Pospíšil, J., Hnilicová, J., Šanderová, H., Barvík, I. and Krásný, L. (2019) The core and holoenzyme forms of RNA polymerase from Mycobacterium smegmatis. J. Bacteriol. 201: e0058318.

Krásný, L. and Gourse, R. L. (2004) An alternative strategy for bacterial ribosome synthesis: Bacillus subtilis rRNA transcription regulation. EMBO J. 23: 4473-4483.

Krásný, L., Tišerová, H., Jonák, J., Rejman, D. and Šanderová, H. (2008) The identity of the transcription +1 position is crucial for changes in gene expression in response to amino acid starvation in Bacillus subtilis. Mol. Microbiol. 69: 42-54.

Kunst, F. and Rapoport, G. (1995) Salt stress is an environmental signal affecting degradative enzyme synthesis in Bacillus subtilis. J. Bacteriol. 177: 2403-2407.

Kusuya, Y., Kurokawa, K., Ishikawa, S., Ogasawara, N. and Oshima, T. (2011) Transcription factor GreA contributes to resolving promoter-proximal pausing of RNA polymerase in Bacillus subtilis cells. J. Bacteriol. 193: 3090-3099.

Laalami, S., Bessières, P., Rocca, A., Zig, L., Nicolas, P. and Putzer, H. (2013) Bacillus subtilis RNase $\mathrm{Y}$ activity in vivo analysed by tiling microarrays. PLoS One 8: e54062.

Laalami, S., Zig, L. and Putzer, H. (2014) Initiation of mRNA decay in bacteria. Cell. Mol. Life Sci. 71: 1799-1828.

Lagesen, K., Ussery, D. W. and Wassenaar, T. M. (2010) Genome update: the 1000th genome - a cautionary tale. Microbiology 156: 603-608.

Langmead, B. and Salzberg, S. (2012) Fast gapped-read alignment with Bowtie 2. Nat. Methods 9: 357-359.

Lee, C. R., Cho, S. H., Yoon, M. J., Peterkofsky, A. and Seok, Y. J. (2007) Escherichia coli enzyme ${ } \mathrm{A}^{\mathrm{Ntr}}$ regulates the $\mathrm{K}^{+}$transporter TrkA. Proc. Natl. Acad. Sci. U. S. A. 104: 4124-4129.

Lee, K., Zhan, X., Gao, J., Qiu, J., Feng, Y., Meganathan, R. et al. (2003) RraA: a protein inhibitor of RNase E activity that globally modulates RNA abundance in E. coli. Cell 114: 623-634. 
Lehnik-Habrink, M., Pförtner, H., Rempeters, L., Pietack, N., Herzberg, C. and Stülke, J. (2010) The RNA degradosome in Bacillus subtilis: identification of CshA as the major RNA helicase in the multiprotein complex. Mol. Microbiol. 77: 958-971.

Lehnik-Habrink, M., Newman, J., Rothe, F. M., Solovyova, A. S., Rodrigues, C., Herzberg, C. et al. (2011a) RNase $\mathrm{Y}$ in Bacillus subtilis: a natively disordered protein that is the functional equivalent of RNase E from Escherichia coli. J. Bacteriol. 193: 5431-5441.

Lehnik-Habrink, M., Schaffer, M., Mäder, U., Diethmaier, C., Herzberg, C. and Stülke, J. (2011b) RNA processing in Bacillus subtilis: identification of targets of the essential RNase Y. Mol. Microbiol. 81: 1459-1473.

Lehnik-Habrink, M., Lewis, R. J., Mäder, U. and Stülke, J. (2012) RNA degradation in Bacillus subtilis: an interplay of essential endo- and exoribonucleases. Mol. Microbiol. 84: 1005-1017.

Lehnik-Habrink, M., Rempeters, L., Kovács, Á. T., Wrede, C., Baierlein, C., Krebber, H. et al. (2013) DEAD-box RNA helicases in Bacillus subtilis have multiple functions and act independently from each other. J. Bacteriol. 195: 534-544.

Leroy, M., Piton, J., Gilet, L., Pellegrini, O., Proux, C., Coppée, J.-Y. et al. (2017) Rae1/YacP, a new endoribonuclease involved in ribosome-dependent mRNA decay in Bacillus subtilis. EMBO J. 36: 1167-1181.

Lin-Chao, S., Chiou, N.-T. and Schuster, G. (2007) The PNPase, exosome and RNA helicases as the building components of evolutionarily-conserved RNA degradation machines. J. Biomed. Sci. 14: $523-532$.

Lin, T. H., Wei, G. T., Su, C. C. and Shaw, G. C. (2012) AdeR, a PucR-Type transcription factor, activates expression of L-Alanine dehydrogenase and is required for sporulation of Bacillus subtilis. J. Bacteriol. 194: 4995-5001.

Linder, P. and Jankowsky, E. (2011) From unwinding to clamping - the DEAD box RNA helicase family. Nat. Rev. Mol. Cell Biol. 12: 505-516.

Linder, P., Lemeille, S. and Redder, P. (2014) Transcriptome-wide analyses of 5'-Ends in RNase J mutants of a gram-positive pathogen reveal a role in RNA maturation, regulation and degradation. PLoS Genet. 10: e1004207.

Liu, B., Deikus, G., Bree, A., Durand, S., Kearns, D. B. and Bechhofer, D. H. (2014) Global analysis of mRNA decay intermediates in Bacillus subtilis wild-type and polynucleotide phosphorylasedeletion strains. Mol. Microbiol. 94: 41-55. 
Liu, B., Kearns, D. B. and Bechhofer, D. H. (2016) Expression of multiple Bacillus subtilis genes is controlled by decay of slrA mRNA from Rho-dependent 3' ends. Nucleic Acids Res. 44: 3364-3372.

López, D., Vlamakis, H. and Kolter, R. (2009) Generation of multiple cell types in Bacillus subtilis. FEMS Microbiol. Rev. 33: 152-163.

López, D. and Kolter, R. (2010) Extracellular signals that define distinct and coexisting cell fates in Bacillus subtilis. FEMS Microbiol. Rev. 34: 134-149.

Luciano, D. J., Vasilyev, N., Richards, J., Serganov, A. and Belasco, J. G. (2017) A novel RNA phosphorylation state enables 5' end-dependent degradation in Escherichia coli. Mol. Cell 67: 4454.

Luttinger, A., Hahn, J. and Dubnau, D. (1996) Polynucleotide phosphorylase is necessary for competence development in Bacillus subtilis. Mol. Microbiol. 19: 343-356.

Maamar, H. and Dubnau, D. (2005) Bistability in the Bacillus subtilis K-state (competence) system requires a positive feedback loop. Mol. Microbiol. 56: 615-624.

Mackie, G. A. (1998) Ribonuclease E is a 5'-end-dependent endonuclease. Nature 395: 720-723.

Mackie, G. A. (2013) RNase E: At the interface of bacterial RNA processing and decay. Nat. Rev. Microbiol. 11: 45-57.

Mäder, U., Zig, L., Kretschmer, J., Homuth, G. and Putzer, H. (2008) mRNA processing by RNases J1 and J2 affects Bacillus subtilis gene expression on a global scale. Mol. Microbiol. 70: 183-196.

Magnuson, R., Solomon, J. and Grossman, A. D. (1994) Biochemical and genetic characterization of a competence pheromone from B. subtilis. Cell 77: 207-216.

Maier, B. (2020) Competence and transformation in Bacillus subtilis. Curr. Issues Mol. Biol. 37: 5776.

Marincola, G., Schäfer, T., Behler, J., Bernhardt, J., Ohlsen, K., Goerke, C. and Wolz, C. (2012) RNase Y of Staphylococcus aureus and its role in the activation of virulence genes. Mol. Microbiol. 85: 817-832.

Marincola, G. and Wolz, C. (2017) Downstream element determines RNase $Y$ cleavage of the saePQRS operon in Staphylococcus aureus. Nucleic Acids Res. 45: 5980-5994.

Mars, R. A. T., Nicolas, P., Ciccolini, M., Reilman, E., Reder, A., Schaffer, M. et al. (2015) Small regulatory RNA-Induced growth rate heterogeneity of Bacillus subtilis. PLOS Genet. 11: e1005046. Martínez-Carranza, E., Barajas, H., Alcaraz, L. D., Servín-González, L., Ponce-Soto, G. Y. and 
Soberón-Chávez, G. (2018) Variability of bacterial essential genes among closely related bacteria: The case of Escherichia coli. Front. Microbiol. 9: 1059.

Mascher, T., Margulis, N. G., Wang, T., Ye, R. W. and Helmann, J. D. (2003) Cell wall stress responses in Bacillus subtilis: The regulatory network of the bacitracin stimulon. Mol. Microbiol. 50: 1591-1604.

Mathy, N., Bénard, L., Pellegrini, O., Daou, R., Wen, T. and Condon, C. (2007) 5'-to-3' exoribonuclease activity in bacteria: role of RNase J1 in rRNA maturation and 5' stability of mRNA. Cell 129: 681-692.

Mathy, N., Hébert, A., Mervelet, P., Bénard, L., Dorléans, A., Li De La Sierra-Gallay, I. et al. (2010) Bacillus subtilis ribonucleases J1 and J2 form a complex with altered enzyme behaviour. Mol. Microbiol. 75: 489-498.

Mechold, U., Fang, G., Ngo, S., Ogryzko, V. and Danchin, A. (2007) Ytql from Bacillus subtilis has both oligoribonuclease and pAp-phosphatase activity. Nucleic Acids Res. 35: 4552-4561.

Meek, D. W. and Hayward, R. S. (1986) Direct evidence for autogenous regulation of the Escherichia coli genes rpoBC in vivo. MGG Mol. Gen. Genet. 202: 500-508.

Meisner, J., Montero Llopis, P., Sham, L. T., Garner, E., Bernhardt, T. G. and Rudner, D. Z. (2013) FtsEX is required for CwlO peptidoglycan hydrolase activity during cell wall elongation in Bacillus subtilis. Mol. Microbiol. 89: 1069-1083.

Miczak, A., Kaberdin, V. R., Wei, C. L. and Lin-Chao, S. (1996) Proteins associated with RNase E in a multicomponent ribonucleolytic complex. Proc. Natl. Acad. Sci. U. S. A. 93: 3865-3869.

Mirouze, N., Desai, Y., Raj, A. and Dubnau, D. (2012) Spo0A P imposes a temporal gate for the bimodal expression of competence in Bacillus subtilis. PLoS Genet. 8: e1002586.

Mirouze, N., Ferret, C., Cornilleau, C. and Carballido-López, R. (2018) Antibiotic sensitivity reveals that wall teichoic acids mediate DNA binding during competence in Bacillus subtilis. Nat. Commun. 9: 5072.

Missiakas, D. M. and Schneewind, O. (2013) Growth and Laboratory Maintenance of Staphylococcus aureus. Curr Protoc Microbiol. Chapter 9: Unit-9C.1.

Mitchell, P., Petfalski, E., Shevchenko, A., Mann, M. and Tollervey, D. (1997) The exosome: A conserved eukaryotic RNA processing complex containing multiple $3^{\prime} \rightarrow 5^{\prime}$ exoribonucleases. Cell 91: 457-466. 
Mohanty, B. K. and Kushner, S. R. (2000) Polynucleotide phosphorylase functions both as a $3^{\prime} \rightarrow$ 5' exonuclease and a poly(A) polymerase in Escherichia coli. Proc. Natl. Acad. Sci. U. S. A. 97: 11966-11971.

Mohanty, B. K. and Kushner, S. R. (2018) Enzymes involved in post-transcriptional RNA metabolism in gram-negative bacteria. Microbiol. Spectr. 6: RWR-0011-2017.

Mohanty, B. K., Agrawal, A. and Kushner, S. R. (2020) Generation of pre-tRNAs from polycistronic operons is the essential function of RNase P in Escherichia coli. Nucleic Acids Res. 48: 2564-2578.

Montero Llopis, P., Jackson, A. F., Sliusarenko, O., Surovtsev, I., Heinritz, J., Emonet, T. and Jacobs-Wagner, C. (2010) Spatial organization of the flow of genetic information in bacteria. Nature 466: 77-81.

Morita, T., Kawamoto, H., Mizota, T., Inada, T. and Aiba, H. (2004) Enolase in the RNA degradosome plays a crucial role in the rapid decay of glucose transporter mRNA in the response to phosphosugar stress in Escherichia coli. Mol. Microbiol. 54: 1063-1075.

Mortazavi, A., Williams, B. A., McCue, K., Schaeffer, L. and Wold, B. (2008) Mapping and quantifying mammalian transcriptomes by RNA-Seq. Nat. Methods 5: 621-628.

Murashko, O. N. and Lin-Chao, S. (2017) Escherichia coli responds to environmental changes using enolasic degradosomes and stabilized DicF sRNA to alter cellular morphology. Proc. Natl. Acad. Sci. 114: E8025-E8034.

Najjar, N. El, Andari, J. El, Kaimer, C., Fritz, G., Rösch, T. C. and Graumann, P. L. (2018) Single molecule tracking of DNA translocases in Bacillus subtilis reveals strikingly different dynamics of SftA, SpollIE, and FtsA. Appl. Environ. Microbiol. 84: e02610-17.

Nakano, M. M., Xia, L. and Zuber, P. (1991) Transcription initiation region of the srfA operon, which is controlled by the comP-comA signal transduction system in Bacillus subtilis. J. Bacteriol. 173: 5487-5493.

Newman, J. A., Hewitt, L., Rodrigues, C., Solovyova, A., Harwood, C. R. and Lewis, R. J. (2011) Unusual, dual endo- and exonuclease activity in the degradosome explained by crystal structure analysis of RNase J1. Structure 19: 1241-1251.

Newman, J. A., Hewitt, L., Rodrigues, C., Solovyova, A. S., Harwood, C. R. and Lewis, R. J. (2012) Dissection of the network of interactions that links RNA processing with glycolysis in the Bacillus subtilis degradosome. J. Mol. Biol. 416: 121-136. 
Nicolas, P., Mäder, U., Dervyn, E., Rochat, T., Leduc, A., Pigeonneau, N. et al. (2012) Conditiondependent transcriptome reveals high-level regulatory architecture in Bacillus subtilis. Science 335: 1103-1106.

Nudler, E., Avetissova, E., Markovtsov, V. and Goldfarb, A. (1996) Transcription processivity: protein-DNA interactions holding together the elongation complex. Science 273: 211-217.

Nudler, E. (2009) RNA Polymerase Active Center: The Molecular Engine of Transcription. Annu. Rev. Biochem. 78: 335-361.

O'Reilly, F. J., Xue, L., Graziadei, A., Sinn, L., Lenz, S., Tegunov, D. et al. (2020) In-cell architecture of an actively transcribing-translating expressome. Science 369: 554-557.

Ogura, M., Yamaguchi, H., Kobayashi, K., Ogasawara, N., Fujita, Y. and Tanaka, T. (2002) Wholegenome analysis of genes regulated by the Bacillus subtilis competence transcription factor ComK. J. Bacteriol. 184: 2344-2351.

Ogura, M. and Asai, K. (2016) Glucose induces ECF sigma factor genes, sigX and sigM, independent of cognate anti-sigma factors through acetylation of CshA in Bacillus subtilis. Front. Microbiol. 7: 1918.

Osaka, N., Kanesaki, Y., Watanabe, M., Watanabe, S., Chibazakura, T., Takada, H. et al. (2020) Novel (p)ppGpp ${ }^{0}$ suppressor mutations reveal an unexpected link between methionine catabolism and GTP synthesis in Bacillus subtilis. Mol. Microbiol. 113: 1155-1169.

Österberg, S., Peso-Santos, T. del and Shingler, V. (2011) Regulation of alternative sigma factor use. Annu. Rev. Microbiol. 65: 37-55.

Oussenko, I. A., Abe, T., Ujiie, H., Muto, A. and Bechhofer, D. H. (2005) Participation of 3'-to-5' exoribonucleases in the turnover of Bacillus subtilis mRNA. J. Bacteriol. 187: 2758-2767.

Pellegrini, O., Nezzar, J., Marchfelder, A., Putzer, H. and Condon, C. (2003) Endonucleolytic processing of CCA-less tRNA precursors by RNase Z in Bacillus subtilis. EMBO J. 22: 4534-4543.

Pettersen, E. F., Goddard, T. D., Huang, C. C., Couch, G. S., Greenblatt, D. M., Meng, E. C. and Ferrin, T. E. (2004) UCSF Chimera - A visualization system for exploratory research and analysis. J. Comput. Chem. 25: 1605-1612.

Pettersson, M. E., Song, A. E., Ae, S., Andersson, D. I. and Berg, O. G. (2009) Evolution of new gene functions: simulation and analysis of the amplification model. Genetica 135: 309-324. 
Phelan, J. P., Kern, A., Ramsey, M. E., Lundt, M. E., Sharma, B., Lin, T. et al. (2019) Genome-wide screen identifies novel genes required for Borrelia burgdorferi survival in its Ixodes tick vector. PloS Pathog. 15: e1007644.

Płociński, P., Macios, M., Houghton, J., Niemiec, E., Płocińska, R., Brzostek, A. et al. (2019) Proteomic and transcriptomic experiments reveal an essential role of RNA degradosome complexes in shaping the transcriptome of Mycobacterium tuberculosis. Nucleic Acids Res. 47: 5892-5905.

Prepiak, P. and Dubnau, D. (2007) A peptide signal for adapter protein-mediated degradation by the AAA+ protease ClpCP. Mol. Cell 26: 639-647.

Price, C. W., Fawcett, P., Cérémonie, H., Su, N., Murphy, C. K. and Youngman, P. (2001) Genomewide analysis of the general stress response in Bacillus subtilis. Mol. Microbiol. 41: 757-774.

Prud'homme-Généreux, A., Beran, R. K., lost, I., Ramey, C. S., Mackie, G. A. and Simons, R. W. (2004) Physical and functional interactions among RNase E, polynucleotide phosphorylase and the cold-shock protein, CsdA: Evidence for a "cold shock degradosome." Mol. Microbiol. 54: 14091421.

Py, B., Higgins, C. F., Krischt, H. M. and Carpousist, A. J. (1996) A DEAD-box RNA helicase in the Escherichia coli RNA degradosome. Nature 381: 169-172.

Qi, Y. and Hulett, F. M. (1998) PhoP P and RNA polymerase $\sigma^{A}$ holoenzyme are sufficient for transcription of Pho regulon promoters in Bacillus subtilis: PhoP P activator sites within the coding region stimulate transcription in vitro. Mol. Microbiol. 28: 1187-1197.

Quentin, Y., Fichant, G. and Denizot, F. (1999) Inventory, assembly and analysis of Bacillus subtilis ABC transport systems. J. Mol. Biol. 287: 467-484.

Rabatinová, A., Šanderová, H., Matějčková, J. J., Korelusová, J., Sojka, L., Barvík, I. et al. (2013) The $\delta$ subunit of RNA polymerase is required for rapid changes in gene expression and competitive fitness of the cell. J. Bacteriol. 195: 2603-2611.

Rahmer, R., Heravi, K. M. and Altenbuchner, J. (2015) Construction of a super-competent Bacillus subtilis 168 using the $\mathrm{P}_{m t \mid A}$-comKS inducible cassette. Front. Microbiol. 6: 1431.

Rasmussen, S., Nielsen, H. B. and Jarmer, H. (2009) The transcriptionally active regions in the genome of Bacillus subtilis. Mol. Microbiol. 73: 1043-1057.

Redder, P. (2018) Molecular and genetic interactions of the RNA degradation machineries in Firmicute bacteria. Wiley Interdiscip. Rev. RNA 9: e1460. 
Redko, Y., Bechhofer, D. H. and Condon, C. (2008) Mini-III, an unusual member of the RNase III family of enzymes, catalyses $23 \mathrm{~S}$ ribosomal RNA maturation in B. subtilis. Mol. Microbiol. 68: 1096-1106.

Redko, Y., Aubert, S., Stachowicz, A., Lenormand, P., Namane, A., Darfeuille, F. et al. (2013) A minimal bacterial RNase J-based degradosome is associated with translating ribosomes. Nucleic Acids Res. 41: 288-301.

Reuß, D. R., Commichau, F. M., Gundlach, J., Zhu, B. and Stülke, J. (2016) The blueprint of a minimal cell: MiniBacillus. Microbiol. Mol. Biol. Rev. 80: 955-987.

Reuß, D. R., Altenbuchner, J., Mäder, U., Rath, H., Ischebeck, T., Sappa, P. K. et al. (2017) Largescale reduction of the Bacillus subtilis genome: Consequences for the transcriptional network, resource allocation, and metabolism. Genome Res. 27: 289-299.

Reuß, D. R., Faßhauer, P., Mroch, P. J., Ul-Haq, I., Koo, B.-M., Pöhlein, A. et al. (2019) Topoisomerase IV can functionally replace all type $1 \mathrm{~A}$ topoisomerases in Bacillus subtilis. Nucleic Acids Res. 47: 5231-5242.

Richards, J., Liu, Q., Pellegrini, O., Celesnik, H., Yao, S., Bechhofer, D. H. et al. (2011) An RNA pyrophosphohydrolase triggers 5 '-exonucleolytic degradation of mRNA in Bacillus subtilis. Mol. Cell 43: 940-949.

Rincón-Tomás, B., González, F. J., Somoza, L., Sauter, K., Madureira, P., Medialdea, T. et al. (2020) Siboglinidae tubes as an additional niche for microbial communities in the Gulf of Cádiz-A microscopical appraisal. Microorganisms 8: 367.

Romero, D. and Palacios, R. (1997) Gene amplification and genomic plasticity in prokaryotes. Annu. Rev. Genet. 31: 91-111.

Ross, W., Thompson, J. F., Newlands, J. T. and Gourse, R. L. (1990) E.coli Fis protein activates ribosomal RNA transcription in vitro and in vivo. EMBO J. 9: 3733-3742.

Rueden, C. T., Schindelin, J., Hiner, M. C., DeZonia, B. E., Walter, A. E., Arena, E. T. and Eliceiri, K. W. (2017) ImageJ2: ImageJ for the next generation of scientific image data. BMC Bioinformatics 18: 529 .

Rustad, T. R., Minch, K. J., Brabant, W., Winkler, J. K., Reiss, D. J., Baliga, N. S. and Sherman, D. R. (2013) Global analysis of mRNA stability in Mycobacterium tuberculosis. Nucleic Acids Res. 41: 509-517. 
Salvo, E., Alabi, S., Liu, B., Schlessinger, A. and Bechhofer, D. H. (2016) Interaction of Bacillus subtilis polynucleotide phosphorylase and RNase Y: STRUCTURAL MAPPING AND EFFECT ON mRNA TURNOVER. J. Biol. Chem. 291: 6655-6663.

Salzberg, L. I., Luo, Y., Hachmann, A.-B., Mascher, T. and Helmann, J. D. (2011) The Bacillus subtilis GntR family repressor YtrA responds to cell wall antibiotics. J. Bacteriol. 193: 5793-5801.

Sambrook, J., Fritsch, E. F. and Maniatis, T. (1989) Molecular cloning: a laboratory manual. 2nd ed. Cold Spring Harbor Laboratory, Cold Spring Harbor, N.Y. 1989

Serizawa, M., Yamamoto, H., Yamaguchi, H., Fujita, Y., Kobayashi, K., Ogasawara, N. and Sekiguchi, J. (2004) Systematic analysis of SigD-regulated genes in Bacillus subtilis by DNA microarray and Northern blotting analyses. Gene 329: 125-136.

Serror, P. and Sonenshein, A. L. (1996) CodY is required for nutritional repression of Bacillus subtilis genetic competence. J. Bacteriol. 178: 5910-5915.

Shahbabian, K., Jamalli, A., Zig, L. and Putzer, H. (2009) RNase Y, a novel endoribonuclease, initiates riboswitch turnover in Bacillus subtilis. EMBO J. 28: 3523-3533.

Shimane, K. and Ogura, M. (2004) Mutational analysis of the helix-turn-helix region of Bacillus subtilis response regulator DegU, and identification of cis-acting sequences for DegU in the aprE and comK promoters. J. Biochem. 136: 387-397.

Shivers, R. P. and Sonenshein, A. L. (2004) Activation of the Bacillus subtilis global regulator CodY by direct interaction with branched-chain amino acids. Mol. Microbiol. 53: 599-611.

Šiková, M., Wiedermannová, J., Převorovský, M., Barvík, I., Sudzinová, P., Kofroňová, O. et al. (2020) The torpedo effect in Bacillus subtilis: RNase J1 resolves stalled transcription complexes. EMBO J. 39: e102500.

Silhavy, T. J., Kahne, D. and Walker, S. (2010) The bacterial cell envelope. Cold Spring Harb. Perspect. Biol. 2: a000414.

Sinderen, D. van, Luttinger, A., Kong, L., Dubnau, D., Venema, G. and Hamoen, L. (1995) comK encodes the competence transcription factor, the key regulatory protein for competence development in Bacillus subtilis. Mol. Microbiol. 15: 455-462.

Smits, W. K., Eschevins, C. C., Susanna, K. A., Bron, S., Kuipers, O. P. and Hamoen, L. W. (2005) Stripping Bacillus: ComK auto-stimulation is responsible for the bistable response in competence development. Mol. Microbiol. 56: 604-614. 
Sojka, L., Kouba, T., Barvík, I., Šanderová, H., Maderová, Z., Jonák, J. and Krásný, L. (2011) Rapid changes in gene expression: DNA determinants of promoter regulation by the concentration of the transcription initiating NTP in Bacillus subtilis. Nucleic Acids Res. 39: 4598-4611.

Steglich, C., Lindell, D., Futschik, M., Rector, T., Steen, R. and Chisholm, S. W. (2010) Short RNA half-lives in the slow-growing marine cyanobacterium Prochlorococcus. Genome Biol. 11: R54.

Strahl, H., Turlan, C., Khalid, S., Bond, P. J., Kebalo, J.-M., Peyron, P. et al. (2015) Membrane recognition and dynamics of the RNA degradosome. PLOS Genet. 11: e1004961.

Sulthana, S., Basturea, G. N. and Deutscher, M. P. (2016) Elucidation of pathways of ribosomal RNA degradation: An essential role for RNase E. RNA 22: 1163-1171.

Tamura, M., Kers, J. A. and Cohen, S. N. (2012) Second-site suppression of RNase E essentiality by mutation of the deaD RNA helicase in Escherichia coli. J. Bacteriol. 194: 1919-1926.

Tamura, M., Honda, N., Fujimoto, H., Cohen, S. N. and Kato, A. (2016) PpsA-mediated alternative pathway to complement RNase E essentiality in Escherichia coli. Arch. Microbiol. 198: 409-421.

Tamura, M., Kageyama, D., Honda, N., Fujimoto, H. and Kato, A. (2017) Enzymatic activity necessary to restore the lethality due to Escherichia coli RNase E deficiency is distributed among bacteria lacking RNase E homologues. PLoS One 12: e0177915.

Tejada-Arranz, A., Crécy-Lagard, V. de and Reuse, H. de (2020) Bacterial RNA Degradosomes: Molecular machines under tight control. Trends Biochem. Sci. 45: 42-57.

Tödter, D., Gunka, K. and Stülke, J. (2017) The highly conserved Asp23 family protein YqhY plays a role in lipid biosynthesis in Bacillus subtilis. Front. Microbiol. 8: 883.

Tomanek, I., Grah, R., Lagator, M., Andersson, A. M. C., Bollback, J. P., Tkačik, G. and Guet, C. C. (2020) Gene amplification as a form of population-level gene expression regulation. Nat. Ecol. Evol. .

Tsai, Y. C., Du, D., Domínguez-Malfavón, L., Dimastrogiovanni, D., Cross, J., Callaghan, A. J. et al. (2012) Recognition of the 70S ribosome and polysome by the RNA degradosome in Escherichia coli. Nucleic Acids Res. 40: 10417-10431.

Tsirigotaki, A., Geyter, J. De, Šoštarić, N., Economou, A. and Karamanou, S. (2017) Protein export through the bacterial Sec pathway. Nat. Rev. Microbiol. 15: 21-36.

Turgay, K., Hahn, J., Burghoorn, J. and Dubnau, D. (1998) Competence in Bacillus subtilis is controlled by regulated proteolysis of a transcription factor. EMBO J. 17: 6730-6738. 
Wach, A. (1996) PCR-synthesis of marker cassettes with long flanking homology regions for gene disruptions in S. cerevisiae. Yeast 12: 259-265.

Wang, W. and Bechhofer, D. H. (1996) Properties of a Bacillus subtilis polynucleotide phosphorylase deletion strain. J. Bacteriol. 178: 2375-2382.

Weber, M. H., Volkov, A. V, Fricke, I., Marahiel, M. A. and Graumann, P. L. (2001) Localization of cold shock proteins to cytosolic spaces surrounding nucleoids in Bacillus subtilis depends on active transcription. J. Bacteriol. 183: 6435-6443.

Weinrauch, Y., Penchev, R., Dubnau, E., Smith, E. and Dubnau, D. (1990) A Bacillus subtilis regulatory gene product for genetic competence and sporulation resembles sensor protein members of the bacterial two-component signal-transduction systems. Genes Dev. 4: 860-872.

Wenzel, M., Kohl, B., Münch, D., Raatschen, N., Albada, H. B., Hamoen, L. et al. (2012) Proteomic response of Bacillus subtilis to lantibiotics reflects differences in interaction with the cytoplasmic membrane. Antimicrob. Agents Chemother. 56: 5749-5757.

Wiedermannová, J., Sudzinová, P., Koval', T., Rabatinová, A., Šanderova, H., Ramaniuk, O. et al. (2014) Characterization of HelD, an interacting partner of RNA polymerase from Bacillus subtilis. Nucleic Acids Res. 42: 5151-5163.

Wölfel, S. (2018) Characterization of a $60 \mathrm{~kb}$ genome amplification in Bacillus subtilis $\Delta r n y$ suppressor mutants. Master thesis. University of Göttingen.

Worrall, J. A. R., Górna, M., Crump, N. T., Phillips, L. G., Tuck, A. C., Price, A. J. et al. (2008) Reconstitution and analysis of the multienzyme Escherichia coli RNA degradosome. J. Mol. Biol. 382: 870-883.

Yadav, T., Carrasco, B., Hejna, J., Suzuki, Y., Takeyasu, K. and Alonso, J. C. (2013) Bacillus subtilis DprA recruits RecA onto single-stranded DNA and mediates annealing of complementary strands coated by SsbB and SsbA. J. Biol. Chem. 288: 22437-22450.

Yan, H., Qin, X., Wang, L. and Chen, W. (2020) Both enolase and the DEAD-box RNA helicase CrhB can form complexes with RNase E in Anabaena sp. PCC 7120. Appl. Environ. Microbiol. 86: e00425-20.

Yang, E., Nimwegen, E. van, Zavolan, M., Rajewsky, N., Schroeder, M., Magnasco, M. and Darnell, J. E. (2003) Decay rates of human mRNAs: Correlation with functional characteristics and sequence attributes. Genome Res. 13: 1863-1872. 
Yao, S. and Bechhofer, D. H. (2010) Initiation of decay of Bacillus subtilis rpsO mRNA by endoribonuclease RNase Y. J. Bacteriol. 192: 3279-3286.

Yao, S., Richards, J., Belasco, J. G. and Bechhofer, D. H. (2011) Decay of a model mRNA in Bacillus subtilis by a combination of RNase $\mathrm{J} 15^{\prime}$ exonuclease and RNase $\mathrm{Y}$ endonuclease activities. $J$. Bacteriol. 193: 6384-6386.

Yoshida, K.-I., Fujita, Y. and Dusko Ehrlich, A. S. (2000) An Operon for a putative ATP-binding cassette transport system involved in acetoin utilization of Bacillus subtilis. J. Bacteriol. 182: 54545461.

Youngman, P. (1990) Use of transposons and integrational vectors for mutagenesis and construction of gene fusions in Bacillus subtilis. In Molecular biological methods for Bacillus. Harwood, C.R. (ed.), Chichester: Wiley, pp. 221-266

Yubero, P. and Poyatos, J. F. (2020) The impact of global transcriptional regulation on bacterial gene order. iScience 23: 101029.

Zhu, B. and Stülke, J. (2018) SubtiWiki in 2018: From genes and proteins to functional network annotation of the model organism Bacillus subtilis. Nucleic Acids Res. 46: D743-D748.

Zhu, X., Liu, D., Singh, A. K., Drolia, R., Bai, X., Tenguria, S. and Bhunia, A. K. (2018) Tunicamycin mediated inhibition of wall teichoic acid affects Staphylococcus aureus and Listeria monocytogenes cell morphology, biofilm formation and virulence. Front. Microbiol. 9: 1352. 


\section{Appendix}

\subsection{Supplementary material}

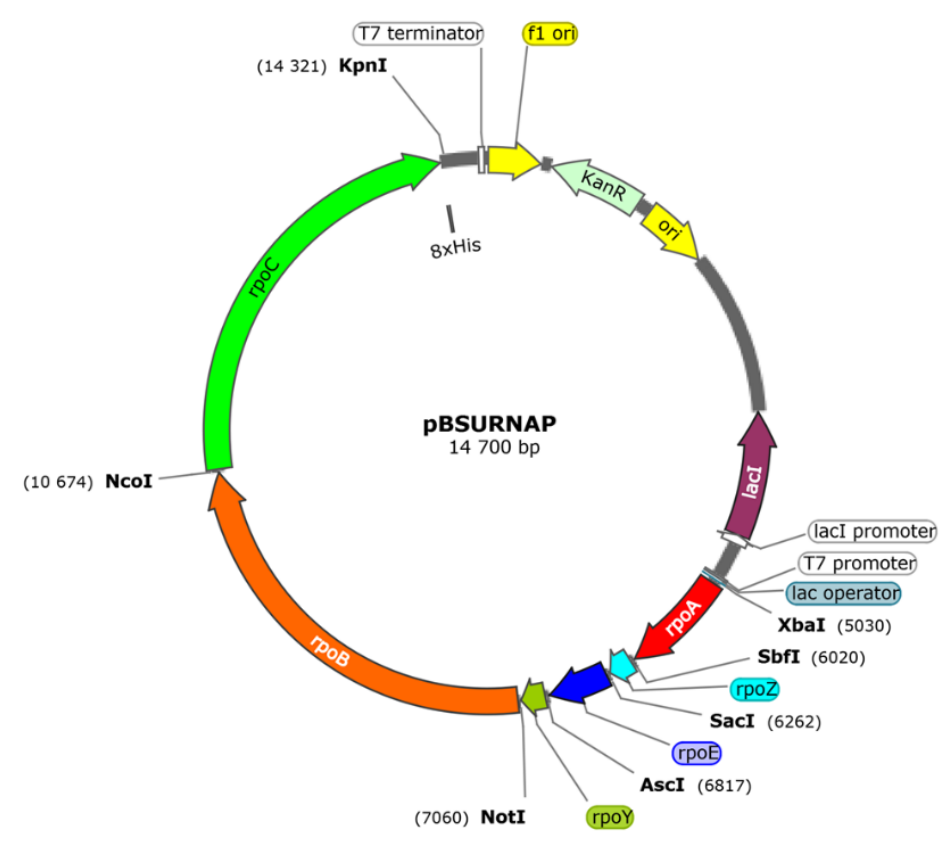

Figure S1: Plasmid map of pBSURNAP used for the expression of the core RNA polymerase.

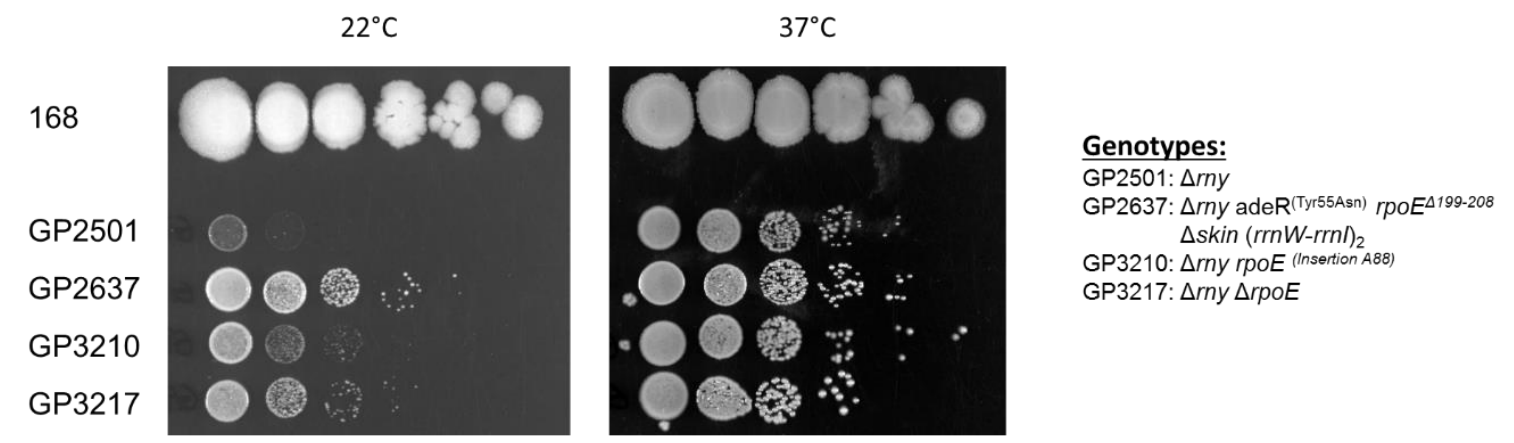

Figure S2: Suppressors of rny with mutations in rpoE and the rny rpoE double mutant show improved growth at $22^{\circ} \mathrm{C}$, but not at $37^{\circ} \mathrm{C}$.

Serial drop dilutions comparing growth of the wild type 168, the rny mutant (GP2501), its derived suppressor mutants evolved at LB agar plates at $22^{\circ} \mathrm{C}$ (GP2637 and GP3210) and the rny rpoE double mutant (GP3217). The pictures were taken after 3 days of incubation at $22^{\circ} \mathrm{C}$ and 1 day of incubation at $37^{\circ} \mathrm{C}$, respectively. 
$37^{\circ} \mathrm{C}$

168

GP2501

GP2636

GP2678

GP3210

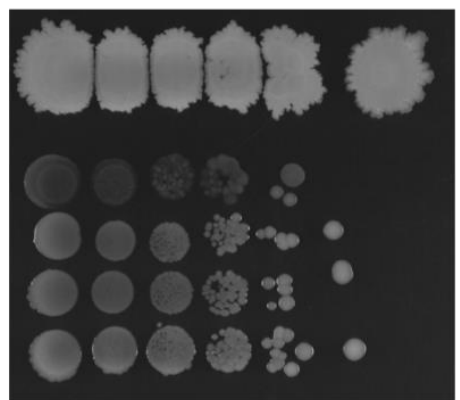

$22^{\circ} \mathrm{C}$

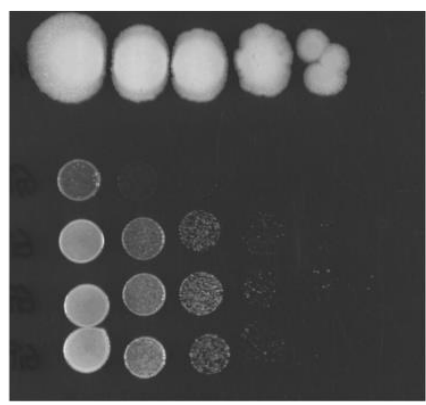

\section{Genotypes:}

GP2501: $\Delta r n y$

GP2636: $\Delta r n y \operatorname{csp} D^{\text {(Trp8stop) }}(r r n W-r r n l){ }_{2}$ GP2678: $\triangle$ rny $\operatorname{cspD} D^{R B S(G G A G G A \rightarrow G G A A G A)}$

GP3217: $\Delta r n y \Delta c s p D$

Figure S3: Suppressors of rny with mutations affecting $\operatorname{cspD}$ and the rny cspD double mutant show improved growth both at $37^{\circ} \mathrm{C}$ and $22^{\circ} \mathrm{C}$.

Serial drop dilutions comparing growth of the wild type 168, the rny mutant (GP2501), its derived suppressor mutants evolved at LB agar plates at $37^{\circ} \mathrm{C}$ (GP2636 and GP2678) and the rny cspD double mutant (GP2615). The pictures were taken after 3 days of incubation at $37^{\circ} \mathrm{C}$ and $22^{\circ} \mathrm{C}$, respectively.

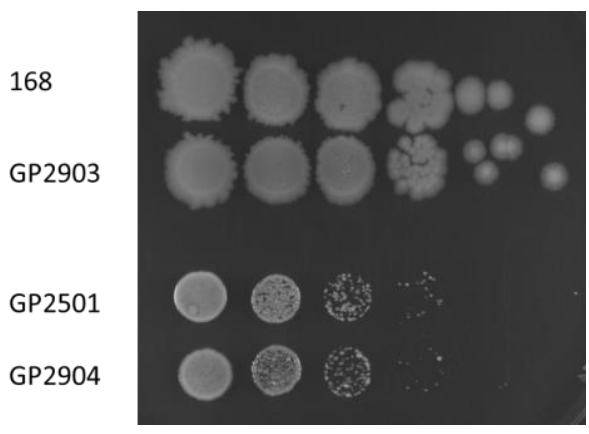

Figure S4: Relocation of rpoA does not affect growth.

Serial drop dilutions comparing growth of the wild type 168, the wild type strain with relocated rpoA GP2903, and their respective rny deletion strains GP2501 and GP2904 on a LB plate at $37^{\circ} \mathrm{C}$. The picture was taken after $18 \mathrm{~h}$ of incubation. 
Table S1. Effect of the rny deletion on the expression of $B$. subtilis genes and operons.

All operons that exhibited an at least eight-fold change upon deletion of rny are shown (and relevant sigma factor genes). In case of differential expression within one operon, the genes not in bold did not met this 8-fold criteria.

\begin{tabular}{|c|c|c|c|}
\hline Transcription unit & Function $^{a}$ & Regulation $^{\mathrm{b}}$ & Fold changes \\
\hline \multicolumn{4}{|c|}{ mRNAs with increased amount in the rny mutant } \\
\hline$y \times k C$ & unknown & SigD, TnrA & 0.010 \\
\hline epr & minor extracellular serine protease, involved in control of swarming motility & SigD, SpoOA, SinR, DegU, ScoC & 0.013 \\
\hline yfmT-S & vanillin dehydrogenase/soluble chemotaxis receptor & SigD & 0.022 \\
\hline $\operatorname{mot} A-B$ & H+-coupled MotA-MotB flagellar stator & SigD & 0.023 \\
\hline hemAT & soluble chemotaxis receptor, heme-containing $\mathrm{O}_{2}$ sensor protein & SigD & 0.026 \\
\hline lytF & major autolysin & SigD, SinR, SlrR & 0.031 \\
\hline hag & flagellin protein & SigD, CodY, ScoC, CsrA & 0.031 \\
\hline glpT-Q & glycerol-3-phosphate permease and diesterase & GlpP, PhoP, CcpA & 0.036 \\
\hline pyrR-P-B-C-AA-AB-K-D-F-E & pyrimidine biosynthesis & PyrR & 0.047 \\
\hline pgdS & gamma-DL-glutamyl hydrolase & SigD & 0.049 \\
\hline pstS-C-A-BA-BB & high-affinity phosphate uptake & PhoP & 0.056 \\
\hline $\operatorname{artP}-Q-R$ & high affinity arginine $A B C$ transporter & YIXR & 0.056 \\
\hline$y v b x$ & putative glycoside hydrolase & & 0.065 \\
\hline$y v b J$ & unknown & & 0.065 \\
\hline lip & extracellular lipase & AbrB & 0.067 \\
\hline ухеK-snaB-yхеM-N-O-sndB-yxeQ & $\mathrm{N}$-acetylcysteine deacetylase & CymR & 0.068 \\
\hline$t / p A-m c p A$ & \multicolumn{2}{|c|}{ membrane-bound chemotaxis receptor, methyl-accepting chemotaxis protein SigD, AbrB } & 0.079 \\
\hline yocC- $D$ & \multicolumn{2}{|l|}{ unknown } & 0.083 \\
\hline ctaO & heme O synthase (minor enzyme) & AbrB & 0.083 \\
\hline lytA-B-C & autolysins & SigD, SinR, YvrHb, SIrR & 0.090 \\
\hline$t / p C$ & \multicolumn{2}{|l|}{ membrane-bound chemotaxis receptor, methyl-accepting chemotaxis protein SigD } & 0.090 \\
\hline mntA-B-C-D & manganese $A B C$ transporter & MntR & 0.093 \\
\hline tuaA/2-A/1-B-C-D-E-F-G-H & biosynthesis of teichuronic acid & PhoP, SigF & 0.096 \\
\hline
\end{tabular}




\begin{tabular}{|c|c|c|c|}
\hline flho-P & flagellar assembly & SigD & 0.102 \\
\hline yvyC-fliD-S-T-A-hpf & flagellar assembly & SigD & 0.109 \\
\hline $\operatorname{sun} A-\operatorname{sun} T-b d b A-y o l J-b d b B$ & sublancin export and processing & Rok, AbrB, Abh, YvrHb, DnaA & 0.114 \\
\hline nat $A-B$ & $\mathrm{Na}^{+} \mathrm{ABC}$ transporter (export) & NatR & 0.114 \\
\hline spoom & sporulation control & SigH, SigW & 0.122 \\
\hline $\operatorname{dgc} W$ & synthesis of c-di-GMP & SigD & 0.122 \\
\hline lipB & extracellular lipase & & 0.123 \\
\hline $\operatorname{sig} D$ & alternative sigma factor & $\begin{array}{l}\text { SigD, Spo0A, SwrA, CodY } \\
\text { DegU }\end{array}$ & 0.430 \\
\hline \multicolumn{4}{|c|}{ mRNAs with decreased amount in the rny mutant } \\
\hline yonP-O-N & SP $\beta$ prophage & & 18.51 \\
\hline sspF & small acid-soluble spore protein & SigG & 18.00 \\
\hline$y h d x$ & unknown & & 16.37 \\
\hline ysnF & general stress protein, survival of ethanol stress & SigB & 15.22 \\
\hline $\operatorname{ssp} B$ & small acid-soluble spore protein & SigG, SpoVt & 14.93 \\
\hline yukJ & unknown & & 14.52 \\
\hline nhax & general stress protein, putative regulator of $n h a C$ & SigB & 14.03 \\
\hline levD-E-F-G-sacC & fructose-specific phosphotransferase system & SigL, LevR, CcpA & 13.30 \\
\hline yhfH & unknown & YIXR & 13.17 \\
\hline$y j b C-s p x$ & general stress proteins, required for survival of salt and paraquate stresses & SigB, SigM, SigW, SigX, PerR & 12.81 \\
\hline ytzE & transcriptional regulator & & 12.21 \\
\hline$f b p B$ & RNA chaperone for $f s r A$, response to iron limitation & Fur & 12.03 \\
\hline yrzF & putative serine/threonine-protein kinase & & 11.73 \\
\hline frlB-O-N-M-frlD-yurJ & Uptake and metabolisms of sugar amines & FrIR, CodY, YlxR & 11.00 \\
\hline corA & general stress protein, similar to magnesium transporter & SigB , LexA & 9.84 \\
\hline yocH & peptidoglycan hydrolase (amidase) & SpoOA, WalR, AbrB & 9.76 \\
\hline speD & S-adenosylmethionine decarboxylase, & $\operatorname{CcpN}$ & 9.72 \\
\hline mreBH-ykpC & cell shape-determining protein/unknown & Sigl, WalR & 9.48 \\
\hline
\end{tabular}




\begin{tabular}{|c|c|c|c|}
\hline slp & small peptidoglycan-associated lipoprotein & & 9.36 \\
\hline yrhH & similar to methyltransferase & SigW,SigM,SigX & 9.05 \\
\hline $\operatorname{tr} B$ & $23 \mathrm{~S}$ rRNA (guanine-N(1)-)-methyltransferase & & 9.03 \\
\hline yuzA & general stress protein & SigB, SigG & 8.61 \\
\hline rsfa & Regulator of SigF-dependent transcription & SigF, SigG & 8.57 \\
\hline bsrA-yrvM & 6S RNA/ tRNA modification enzyme & & 8.55 \\
\hline$y q h B$ & similar to magnesium exporter, general stress protein & SigB, LexA & 8.42 \\
\hline $\operatorname{sig} F$ & sporulation-specific sigma factor & $\begin{array}{l}\text { SigH, SigF, SigG, AbrB, SinR } \\
\text { Spo0A }\end{array}$ & 4.23 \\
\hline $\operatorname{sig} G$ & sporulation-specific sigma factor & SigF, SigG, AbrB, SinR, SpoOA & 4.68 \\
\hline
\end{tabular}

${ }^{a}$ Information was taken from SubtiWiki database (Zhu and Stülke, 2018)

${ }^{\mathrm{b}}$ The housekeeping sigma factor SigA is not listed as a regulator 
Table S2: Genes with (partially) restored expression in the suppressor mutant

Numbers of reads corresponding to the listed genes are shown. Essential genes are highlighted in blue

\begin{tabular}{|c|c|c|c|c|}
\hline$\#$ & Gene & $\begin{array}{c}\text { Wild } \\
\text { type } \\
168 \\
\end{array}$ & $\begin{array}{c}\text { GP2501 } \\
\Delta r n y\end{array}$ & $\begin{array}{c}\text { GP2518 } \\
\Delta r n y \Delta g r e A \\
(r r n W-r r n l)_{2}\end{array}$ \\
\hline \multicolumn{5}{|c|}{ Genes upregulated in the rny mutant } \\
\hline 1 & yon $N$ & 14 & 265 & 107 \\
\hline 2 & $\operatorname{ssp} F$ & 33 & 594 & 245 \\
\hline 3 & levE & 11 & 174 & 23 \\
\hline 4 & $\operatorname{ssp} B$ & 13 & 199 & 20 \\
\hline 5 & yukJ & 211 & 3068 & 1378 \\
\hline 6 & $f r l B$ & 49 & 714 & 100 \\
\hline 7 & frlO & 17 & 221 & 25 \\
\hline 8 & levF & 17 & 191 & 23 \\
\hline 9 & levG & 20 & 173 & 36 \\
\hline 10 & $y u z A$ & 12 & 103 & 48 \\
\hline 11 & $r s f A$ & 12 & 106 & 19 \\
\hline 12 & $x t m B$ & 752 & 5972 & 259 \\
\hline 13 & $x k d E$ & 733 & 5440 & 222 \\
\hline 14 & $x \operatorname{tmA}$ & 433 & 3171 & 128 \\
\hline 15 & $\operatorname{roc} A$ & 1077 & 7435 & 529 \\
\hline 16 & yonH & 13 & 86 & 28 \\
\hline 17 & $\operatorname{trpC}$ & 31 & 197 & 89 \\
\hline 18 & qdol & 67 & 403 & 127 \\
\hline 19 & yonJ & 24 & 143 & 34 \\
\hline 20 & $y f h K$ & 63 & 371 & 150 \\
\hline 21 & yfiu & 50 & 277 & 83 \\
\hline 22 & $\operatorname{trp} B$ & 80 & 433 & 215 \\
\hline 23 & opuCA & 234 & 1219 & 222 \\
\hline 24 & veg & 1810 & 9193 & 3024 \\
\hline 25 & $y x a H$ & 86 & 434 & 191 \\
\hline 26 & $x k d U$ & 143 & 687 & 38 \\
\hline 27 & $y d a D$ & 58 & 280 & 129 \\
\hline 28 & $y k g A$ & 32 & 151 & 64 \\
\hline 29 & yrkF & 12 & 58 & 19 \\
\hline 30 & $p k s D$ & 25 & 112 & 30 \\
\hline 31 & yomV & 23 & 103 & 47 \\
\hline 32 & opuCB & 109 & 474 & 80 \\
\hline 33 & $x k z A$ & 72 & 313 & 14 \\
\hline 34 & $o x d C$ & 94 & 401 & 130 \\
\hline 35 & $y j g D$ & 28 & 117 & 58 \\
\hline 36 & $y b b A$ & 278 & 1161 & 334 \\
\hline 37 & yrkH & 28 & 118 & 47 \\
\hline 38 & $x k d R$ & 82 & 341 & 16 \\
\hline 39 & ypzA & 30 & 125 & 46 \\
\hline 40 & gerW & 25 & 101 & 27 \\
\hline
\end{tabular}




\begin{tabular}{|c|c|c|c|c|}
\hline 41 & $x k d M$ & 558 & 2273 & 120 \\
\hline 42 & $x k d S$ & 93 & 377 & 14 \\
\hline 43 & $x k d Q$ & 305 & 1222 & 61 \\
\hline 44 & $y k z L$ & 363 & 1442 & 62 \\
\hline 45 & feuA & 656 & 2594 & 628 \\
\hline 46 & $x k d F$ & 749 & 2930 & 117 \\
\hline 47 & xepA & 290 & 1121 & 39 \\
\hline 48 & spollAA & 13 & 51 & 21 \\
\hline 49 & $x k d W$ & 114 & 437 & 17 \\
\hline 50 & $b a c B$ & 51 & 196 & 88 \\
\hline 51 & youA & 31 & 117 & 43 \\
\hline 52 & $x k d G$ & 914 & 3477 & 139 \\
\hline 53 & yomw & 18 & 69 & 19 \\
\hline 54 & орисC & 165 & 618 & 103 \\
\hline 55 & opuCD & 169 & 628 & 98 \\
\hline 56 & yon $B$ & 48 & 180 & 51 \\
\hline 57 & $x k d V$ & 534 & 1977 & 77 \\
\hline 58 & $y k z l$ & 15 & 55 & 25 \\
\hline 59 & speA & 4381 & 16034 & 6933 \\
\hline 60 & feuB & 446 & 1623 & 484 \\
\hline 61 & murAA & 39336 & 142509 & 56216 \\
\hline 62 & $x k d H$ & 350 & 1269 & 59 \\
\hline 63 & $x k d P$ & 247 & 888 & 64 \\
\hline 64 & $\operatorname{roc} D$ & 539 & 1933 & 455 \\
\hline 65 & spollAB & 25 & 88 & 44 \\
\hline 66 & yomU & 40 & 141 & 46 \\
\hline 67 & yist & 40 & 140 & 65 \\
\hline 68 & $x k d K$ & 1439 & 5052 & 229 \\
\hline 69 & $x k d T$ & 263 & 921 & 45 \\
\hline 70 & yomX & 25 & 86 & 27 \\
\hline 71 & $x h / B$ & 155 & 539 & 19 \\
\hline 72 & yonA & 30 & 104 & 31 \\
\hline 73 & feuc & 419 & 1437 & 488 \\
\hline 74 & $x l y A$ & 795 & 2716 & 137 \\
\hline 75 & $x k d l$ & 461 & 1562 & 55 \\
\hline 76 & yerD & 52 & 174 & 79 \\
\hline 77 & bacc & 69 & 233 & 106 \\
\hline 78 & $x k d O$ & 1611 & 5392 & 722 \\
\hline 79 & $x k d J$ & 364 & 1202 & 59 \\
\hline 80 & $p k s E$ & 43 & 140 & 29 \\
\hline 81 & yobo & 62 & 201 & 64 \\
\hline 82 & yonD & 65 & 203 & 72 \\
\hline 83 & $x k d N$ & 295 & 863 & 51 \\
\hline 84 & yonF & 15 & 45 & 17 \\
\hline 85 & azoR2 & 407 & 1181 & 518 \\
\hline 86 & $y d d J$ & 41 & 115 & 41 \\
\hline
\end{tabular}




\begin{tabular}{|c|c|c|c|c|}
\hline 87 & yonc & 26 & 73 & 21 \\
\hline 88 & yorG & 37 & 104 & 30 \\
\hline 89 & gmuA & 277 & 766 & 53 \\
\hline 90 & yorf & 30 & 83 & 14 \\
\hline 91 & yisk & 335 & 922 & 408 \\
\hline 92 & $r o c B$ & 119 & 323 & 56 \\
\hline 93 & $y k z M$ & 239 & 646 & 35 \\
\hline 94 & yono & 16 & 43 & 12 \\
\hline 95 & spollT & 4930 & 13052 & 5899 \\
\hline 96 & $g m u D$ & 1313 & 3471 & 215 \\
\hline 97 & $g / t B$ & 936 & 2468 & 987 \\
\hline 98 & $x h / A$ & 248 & 651 & 21 \\
\hline 99 & yomE & 18 & 46 & 12 \\
\hline 100 & yybF & 464 & 1212 & 436 \\
\hline 101 & yonE & 35 & 87 & 41 \\
\hline 102 & $\operatorname{tag} B$ & 1918 & 4643 & 1720 \\
\hline 103 & yos $P$ & 51 & 121 & 38 \\
\hline 104 & $\operatorname{tag} A$ & 2932 & 6995 & 2221 \\
\hline 105 & $n r d E B$ & 40 & 94 & 38 \\
\hline 106 & $y q g Y$ & 282 & 659 & 325 \\
\hline 107 & spoVG & 2028 & 4548 & 1825 \\
\hline 108 & cw/S & 222 & 495 & 118 \\
\hline 109 & $b a c D$ & 193 & 429 & 195 \\
\hline 110 & уотм & 16 & 36 & 16 \\
\hline 111 & $b d h A$ & 5374 & 11288 & 2499 \\
\hline 112 & opuD & 2318 & 4837 & 2384 \\
\hline 113 & galM & 80 & 165 & 79 \\
\hline 114 & $g m u R$ & 749 & 1533 & 212 \\
\hline 115 & yorl & 24 & 48 & 22 \\
\hline \multicolumn{5}{|c|}{ Genes downregulated in the rny mutant } \\
\hline 1 & artP & 489 & 27 & 57 \\
\hline 2 & $s n d B$ & 836 & 68 & 265 \\
\hline 3 & $y v y C$ & 447 & 39 & 94 \\
\hline 4 & fliD & 4264 & 374 & 777 \\
\hline 5 & yxeQ & 935 & 103 & 319 \\
\hline 6 & spoom & 7850 & 954 & 2678 \\
\hline 7 & epsD & 185 & 25 & 52 \\
\hline 8 & $\operatorname{cyd} A$ & 83 & 13 & 81 \\
\hline 9 & $n r g A$ & 404 & 63 & 359 \\
\hline 10 & $x p t$ & 378 & 68 & 141 \\
\hline 11 & epsN & 61 & 12 & 28 \\
\hline 12 & $y w / D$ & 232 & 45 & 132 \\
\hline 13 & $y x e R$ & 1218 & 237 & 478 \\
\hline 14 & yteJ & 2911 & 579 & 1208 \\
\hline 15 & $q d o R$ & 1982 & 436 & 1148 \\
\hline
\end{tabular}




\begin{tabular}{|c|c|c|c|c|}
\hline 16 & $s k f B$ & 74 & 17 & 35 \\
\hline 17 & $y x j l$ & 781 & 190 & 424 \\
\hline 18 & yvav & 1228 & 311 & 757 \\
\hline 19 & $t s a C$ & 1244 & 320 & 683 \\
\hline 20 & $y j o B$ & 2355 & 606 & 1312 \\
\hline 21 & $p u c R$ & 235 & 64 & 173 \\
\hline 22 & $n a s B$ & 43 & 13 & 41 \\
\hline 23 & сурА & 101 & 30 & 107 \\
\hline 24 & exoA & 454 & 138 & 402 \\
\hline 25 & $y b a E$ & 2328 & 739 & 1821 \\
\hline 26 & $y d g G$ & 94 & 30 & 73 \\
\hline 27 & $h m p$ & 92 & 29 & 86 \\
\hline 28 & yqas & 45 & 15 & 163 \\
\hline 29 & hisz & 38 & 13 & 47 \\
\hline 30 & yqaT & 46 & 16 & 131 \\
\hline 31 & $y q b B$ & 45 & 15 & 72 \\
\hline 32 & yоуA & 74 & 25 & 61 \\
\hline 33 & hisD & 63 & 22 & 58 \\
\hline 34 & comFA & 142 & 52 & 146 \\
\hline 35 & hisA & 82 & 31 & 97 \\
\hline 36 & hutU & 98 & 37 & 95 \\
\hline 37 & spollB & 42 & 16 & 42 \\
\hline 38 & fra & 559 & 216 & 551 \\
\hline 39 & $c y d B$ & 81 & 31 & 77 \\
\hline 40 & hisF & 102 & 40 & 100 \\
\hline 41 & $p h o D$ & 75 & 31 & 65 \\
\hline 42 & prol & 584 & 244 & 1099 \\
\hline 43 & $y w p B$ & 2387 & 999 & 2194 \\
\hline 44 & hutl & 111 & 47 & 103 \\
\hline 45 & hisH & 39 & 16 & 53 \\
\hline 46 & fos $B$ & 352 & 150 & 302 \\
\hline 47 & alat & 4956 & 2120 & 5504 \\
\hline 48 & $y c / G$ & 142 & 61 & 127 \\
\hline 49 & leuB & 61 & 26 & 56 \\
\hline 50 & yqaR & 73 & 32 & 128 \\
\hline 51 & bofA & 235 & 102 & 252 \\
\hline 52 & $c y d C$ & 220 & 96 & 211 \\
\hline 53 & $s p s B$ & 26 & 11 & 27 \\
\hline 54 & alaR & 1460 & 647 & 1733 \\
\hline 55 & $y r p G$ & 46 & 20 & 68 \\
\hline 56 & $f n r$ & 391 & 175 & 562 \\
\hline 57 & trmFO & 3176 & 1438 & 3014 \\
\hline 58 & $g a p B$ & 268 & 130 & 294 \\
\hline 59 & hisB & 37 & 18 & 39 \\
\hline 60 & $y q b A$ & 45 & 22 & 100 \\
\hline 61 & $s p s G$ & 39 & 19 & 41 \\
\hline
\end{tabular}




\subsection{Bacterial strains, plasmids and oligonucleotides}

Table S3: Bacterial strains used in this study

\begin{tabular}{|c|c|c|}
\hline Strain & Genotype $^{c}$ & Source ${ }^{a}$ \\
\hline \multicolumn{3}{|l|}{ B. subtilis } \\
\hline 168 & $\operatorname{trpC2}$ & Laboratory collection \\
\hline BSB1 & Wild type & Nicolas et al., 2012 \\
\hline BKE30420 & $\operatorname{trpC2} \Delta y \operatorname{trE}:: e r m C$ & Koo et al., 2017 \\
\hline BKE30430 & $\operatorname{trpC2} \Delta y \operatorname{trD}:: e r m C$ & Koo et al., 2017 \\
\hline BKE30440 & $\operatorname{trpC2} \Delta y \operatorname{trC}:: \operatorname{ermC}$ & Koo et al., 2017 \\
\hline BKE30450 & $\operatorname{trpC2} \Delta y \operatorname{trB}:: e r m C$ & Koo et al., 2017 \\
\hline BP351 & $\operatorname{trp} C 2 \Delta g r e A:: c a t$ & F. Commichau \\
\hline CCB434 & $\Delta r n j A:: s p c$ & Figaro et al., 2013 \\
\hline CCB441 & $\Delta r n y:: s p c$ & Figaro et al., 2013 \\
\hline DK1042 & $\mathrm{com}^{\mathrm{Q} 12 L}$ & Konkol et al., 2013 \\
\hline LK633 & MO1099 rpoE::aphA3 amyE::m/s & Rabatinová et al., 2013 \\
\hline LK1098 & $\Delta r p o E:: a p h A 3$ & LK633 $\rightarrow$ BSB1 \\
\hline PG389 & amyE::P $P_{c o m G}-l a c Z-g f p-c a t$ & Gamba et al., 2015 \\
\hline PG10 b & $y v c A::\left(P_{m t \mid A}-c o m K S\right)$ & Reuß et al., 2017 \\
\hline GP811 & $\begin{array}{l}\operatorname{trpC2} \Delta \text { gudB::cat rocG::Tn10 spc amyE::(gltA-lacZ } \\
\text { aphA3) } \triangle a n s R:: \text { tet }\end{array}$ & Flórez et al., 2011 \\
\hline GP1152 & $\operatorname{trpC2} \triangle a n s R:: t e t R$ & $\mathrm{GP} 811 \rightarrow 168$ \\
\hline GP1748 & $\operatorname{trp} C 2 \triangle p n p A:: a p h A 3$ & Cascante-Estepa et al., 2016 \\
\hline GP2155 & $\operatorname{trpC2} \triangle n r n A:: a p h A 3$ & $\mathrm{LFH} \rightarrow 168$ \\
\hline GP2501 ${ }^{d}$ & $\operatorname{trp} C 2 \Delta r n y:: s p c$ & CCB441 $\rightarrow 168$ \\
\hline GP2503 ${ }^{d}$ & $\operatorname{trpC2} \Delta r n y:: s p c$ greA (C374T - Ser125Leu) $(r r n W-r r n l)_{2}$ & Evolution of GP2501 at $22^{\circ} \mathrm{C}$ \\
\hline GP2504 & $\operatorname{trpC2} \Delta r n y:: s p c$ greA (G169T - Glu57Stop) & Evolution of GP2501 at $22^{\circ} \mathrm{C}$ \\
\hline GP2506 & $\operatorname{trpC2} \Delta r n j A:: s p c$ & CCB434 $\rightarrow 168$ \\
\hline GP2518 ${ }^{d}$ & $\operatorname{trpC2} \Delta$ greA::cat $\Delta r n y:: s p c(r r n W-r r n l)_{2}$ & $\begin{array}{l}\text { Evolution of GP2628 on LB } \\
\text { agar at } 37^{\circ} \mathrm{C}\end{array}$ \\
\hline GP2524 & $\operatorname{trpC2} \Delta r n y:: e r m C$ & $\mathrm{LFH} \rightarrow 168$ \\
\hline GP2525 & $\operatorname{trpC2}$ greA-3xflag spc & pGP2542 $\rightarrow 168$ \\
\hline GP2529 & $\operatorname{trpC2} \Delta r n y:: e r m C$ greA-3xflag spc & GP2524 $\rightarrow$ GP2525 \\
\hline GP2538 & $\operatorname{trpC2} \Delta r n y:: e r m C$ greA (Insertion A406)-3xflag spc & Evolution of GP2529 at $22^{\circ} \mathrm{C}$ \\
\hline GP2539 & $\operatorname{trpC2} \Delta r n y:: e r m C$ greA (Deletion A66)-3xflag spc & Evolution of GP2529 at $22^{\circ} \mathrm{C}$ \\
\hline
\end{tabular}




\begin{tabular}{|c|c|c|}
\hline GP2542 & $\operatorname{trp} C 2 \Delta r e c A:: s p c$ & Reuß et al., 2019 \\
\hline GP2559 & $\operatorname{coml}^{Q 12 L} \Delta y m d B:: c a t$ & Kampf et al., 2018 \\
\hline GP2612 & $\operatorname{trpC2} \triangle g r e A:: a p h A 3$ & LFH $\rightarrow 168$ \\
\hline GP2614 & 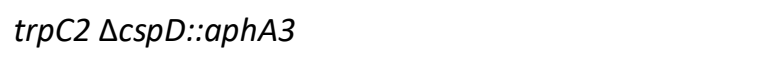 & $\mathrm{LFH} \rightarrow 168$ \\
\hline GP2615 & $\operatorname{trpC2} \Delta c s p D:: a p h A 3 \Delta r n y:: s p c$ & GP2501 $\rightarrow$ GP2614 \\
\hline GP2618 & $\operatorname{trpC2}$ yvcA-P $P_{m t / A}$-comKS-ermC-hisl & $\mathrm{LFH} \rightarrow 168$ \\
\hline GP2620 & $\operatorname{trpC2}$ yvcA-P $P_{m t \mid A}$-comKS-cat-hisl & LFH $\rightarrow 168$ \\
\hline GP2621 & $\operatorname{trpC2}$ yvcA-P $P_{m t / A}$-comKS-ermC-hisl $\triangle p n p A:: a p h A 3$ & GP1748 $\rightarrow$ GP2618 \\
\hline GP2624 & $\operatorname{trpC2}$ yvcA-P $P_{m t / A}$-comKS-ermC-hisl $\Delta r n y:: s p c$ & GP2501 $\rightarrow$ GP2618 \\
\hline GP2626 & $\operatorname{trpC2}$ yvcA-P $P_{m t \mid A}$-comKS-ermC-hisl $\Delta r n j A:: s p c$ & GP2506 $\rightarrow$ GP2618 \\
\hline GP2628d & $\operatorname{trp} C 2 \Delta g r e A:: c a t \Delta r n y:: s p c$ & BP351 + GP2501 $\rightarrow 168$ \\
\hline GP2630 & $\operatorname{trpC}$ amyE::P ${ }_{\text {comG }}-l a c Z-g f p-c a t$ & PG389 $\rightarrow 168$ \\
\hline GP2636 ${ }^{d}$ & $\operatorname{trp} C 2 \Delta r n y:: s p c \operatorname{cspD}(\mathrm{G} 23 \mathrm{~A}-\mathrm{Trp} 8 \mathrm{Stop})(r r n W-r r n l)_{2}$ & $\begin{array}{l}\text { Evolution of GP2501 on LB } \\
\text { agar at } 37^{\circ} \mathrm{C}\end{array}$ \\
\hline GP2637 ${ }^{d}$ & $\begin{array}{l}\operatorname{trpC2} \Delta r n y:: s p c \text { adeR (T163A - Tyr55Asn) } \\
\text { rpoE- } \Delta 199-208 \Delta \text { skin (rrnW-rrnl) }\end{array}$ & $\begin{array}{l}\text { Evolution of GP2501 on LB } \\
\text { agar at } 22^{\circ} \mathrm{C}\end{array}$ \\
\hline GP2640 & $\operatorname{trpC2} \Delta f t s H:: a p h A 3$ & $\mathrm{LFH} \rightarrow 168$ \\
\hline GP2641 & $\operatorname{trp} C 2 \Delta y \operatorname{trA}:: s p c$ & $\mathrm{LFH} \rightarrow 168$ \\
\hline GP2643 & $\operatorname{trpC2} \triangle \mathrm{comEC::spc}$ & $\mathrm{LFH} \rightarrow 168$ \\
\hline GP2644 & $\operatorname{trpC2} \triangle \operatorname{deg} U:: a p h A 3$ & $\mathrm{LFH} \rightarrow 168$ \\
\hline GP2646 & $\operatorname{trpC2} \triangle y \operatorname{trGABCDEF::ermC}$ & $\mathrm{LFH} \rightarrow 168$ \\
\hline GP2647 & $\operatorname{trpC2} \Delta y \operatorname{trA}:: e r m C$ & $\mathrm{LFH} \rightarrow 168$ \\
\hline GP2652 & $\operatorname{trpC2}$ yvcA-P $P_{m t / A}-c o m K S-c a t-h i s l \Delta f t s H:: a p h A 3$ & GP2640 $\rightarrow$ GP2620 \\
\hline GP2653 & $\operatorname{trpC2}$ yvcA-P $P_{m t / A}-c o m K S$-cat-hisl $\Delta n r n A:: a p h A 3$ & GP2155 $\rightarrow$ GP2620 \\
\hline GP2654 & $\operatorname{trpC2}$ yvcA-P $P_{m t / A}-c o m K S$-cat-hisl $\Delta g r e A:: a p h A 3$ & GP2612 $\rightarrow$ GP2620 \\
\hline GP2655 & $\operatorname{trpC2}$ yvcA-P $P_{m t \mid A}-c o m K S$-cat-hisl $\Delta y \operatorname{trA}:: s p c$ & GP2641 $\rightarrow$ GP2620 \\
\hline GP2659 & $\operatorname{trpC2}$ yvcA-P $P_{m t / A}-c o m K S$-cat-hisl $\triangle$ comEC::spc & GP2643 $\rightarrow$ GP2620 \\
\hline GP2660 & $\operatorname{trpC2}$ yvcA-P $P_{m t \mid A}-c o m K S-c a t-h i s l \Delta d e g U:: a p h A 3$ & GP2644 $\rightarrow$ GP2620 \\
\hline GP2664 & 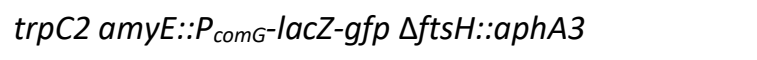 & GP2640 $\rightarrow$ GP2630 \\
\hline GP2665 & $\operatorname{trpC2}$ amyE::P comG - lacZ-gfp $\triangle n r n A:: a p h A 3$ & GP2155 $\rightarrow$ GP2630 \\
\hline GP2666 & 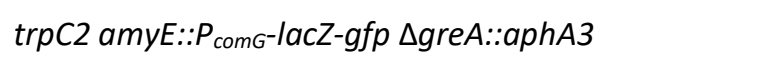 & $\mathrm{GP} 2612 \rightarrow \mathrm{GP} 2630$ \\
\hline GP2667 & $\operatorname{trpC2}$ amyE::P $P_{\text {comG }}-l a c Z-g f p \Delta y \operatorname{trA}:: s p c$ & GP2641 $\rightarrow$ GP2630 \\
\hline GP2671 & $\operatorname{trpC2}$ amyE::P $P_{\text {comG }}-$ lacZ-gfp $\Delta$ comEC::spc & GP2643 $\rightarrow$ GP2630 \\
\hline GP2672 & $\operatorname{trpC2}$ amyE::P comG -lacZ-gfp $\triangle$ degU::aphA3 & GP2644 $\rightarrow$ GP2630 \\
\hline GP2678 & $\operatorname{trpC2} \Delta r n y:: s p c$ RBS of $\operatorname{csp} D(G G A G G A \rightarrow G G A A G A)$ & $\begin{array}{l}\text { Evolution of GP2501 on LB } \\
\text { agar at } 37^{\circ} \mathrm{C}\end{array}$ \\
\hline GP2700 & $\operatorname{trpC2} \Delta y \operatorname{trF}:: c a t$ & $\mathrm{LFH} \rightarrow 168$ \\
\hline GP2901 & trpC2 rae1 (insertion T33) & pGP2826 $\rightarrow 168$ \\
\hline
\end{tabular}




\begin{tabular}{|c|c|c|}
\hline GP2902 & $\operatorname{trpC2} d g k-r p o A-c a t-y a a H$ & $\mathrm{LFH} \rightarrow 168$ \\
\hline GP2903 & $\operatorname{trpC2}$ dgk-rpoA-cat-yaaH $\Delta r p o A:: a p h A 3$ & LFH $\rightarrow 2902$ \\
\hline GP2904 & $\operatorname{trpC2}$ dgk-rpoA-cat-yaaH $\Delta r p o A:: a p h A 3 \Delta r n y:: s p c$ & GP2501 $\rightarrow$ GP2903 \\
\hline GP2907 & $\operatorname{trpC2}$ rael $\mathrm{P}_{\text {alf4- }}$ gfp-ermC sigH & $\mathrm{LFH} \rightarrow 168$ \\
\hline GP2909 & $\begin{array}{l}\operatorname{trpC2} \text { dgk-rpoA-cat-yaaH } \triangle r p o A:: a p h A 3 \text { (rae1 } \mathrm{P}_{\text {alf4- }} \\
\text { gfp-ermC sigH) }\end{array}$ & GP2907 $\rightarrow$ GP2903 \\
\hline GP2910 & $\begin{array}{l}\operatorname{trpC2} \text { dgk-rpoA-cat-yaaH } \Delta r p o A:: a p h A 3 \text { (rae1 } \mathrm{P}_{\text {alf4- }} \\
\text { gfp-ermC sigH) } \Delta r n y:: s p c\end{array}$ & GP2501 $\rightarrow 2909$ \\
\hline GP2912 d & $\begin{array}{l}\operatorname{trpC2} \text { dgk-rpoA-cat-yaaH } \Delta r p o A:: a p h A 3 \Delta r n y:: s p c \text { rpoC } \\
\text { (G263A - Arg88His) } \Delta \text { skin trnSL-Val1 (bp55T -> C) }\end{array}$ & $\begin{array}{l}\text { Evolution of GP2904 on LB } \\
\text { agar at } 37^{\circ} \mathrm{C}\end{array}$ \\
\hline GP2913 ${ }^{d}$ & $\begin{array}{l}\operatorname{trpC2} \text { dgk-rpoA-cat-yaaH } \Delta r p o A:: a p h A 3 \text { (rae1 } \mathrm{P}_{\text {alf4- }} \\
\text { gfp-ermC sigH) } \Delta r n y:: s p c \text { rpoB (G3160T - Gly1054Cys) }\end{array}$ & $\begin{array}{l}\text { Evolution of GP2910 on LB } \\
\text { agar at } 37^{\circ} \mathrm{C}\end{array}$ \\
\hline GP2915 & $\begin{array}{l}\Delta s k i n \\
\operatorname{trpC2} \text { dgk-rpoA-cat-yaaH } \Delta r p o A:: a p h A 3 \text { (rae1 } \mathrm{P}_{a l f 4^{-}} \\
\text {gfp-ermC sigH) } \Delta r n y:: s p c \text { rpoC (G134A - Gly45Asp) }\end{array}$ & $\begin{array}{l}\text { Evolution of GP2910 on LB } \\
\text { agar at } 37^{\circ} \mathrm{C}\end{array}$ \\
\hline GP3186 & $\operatorname{trp} C 2 \triangle y \operatorname{trGABCDE::ermC}$ & $\mathrm{LFH} \rightarrow 168$ \\
\hline GP3187 & $\operatorname{trpC2} \Delta y \operatorname{trF}:: c a t \Delta y \operatorname{trA}:: e r m C$ & GP2647 $\rightarrow$ GP2700 \\
\hline GP3188 & $\operatorname{trp} C 2 \Delta y \operatorname{tr} B$ & pDR244 $\rightarrow$ BKE30450 \\
\hline GP3189 & $\operatorname{trpC2} \Delta y \operatorname{trC}$ & pDR244 $\rightarrow$ BKE30440 \\
\hline GP3190 & $\operatorname{trp} C 2 \Delta y \operatorname{tr} D$ & pDR244 $\rightarrow$ BKE30430 \\
\hline GP3191 & $\operatorname{trp} C 2 \Delta y \operatorname{tr} E$ & pDR244 $\rightarrow$ BKE30420 \\
\hline GP3193 & $\operatorname{trpC2} \Delta y \operatorname{trA}:: e r m C \Delta y \operatorname{tr} B$ & LFH $\rightarrow$ GP3188 \\
\hline GP3194 & $\operatorname{trpC2} \Delta y \operatorname{trA}:: e r m C \Delta y \operatorname{trC}$ & LFH $\rightarrow$ GP3189 \\
\hline GP3195 & $\operatorname{trpC2} \Delta y \operatorname{trA}:: e r m C \Delta y \operatorname{tr} D$ & GP2647 $\rightarrow$ GP3190 \\
\hline GP3196 & $\operatorname{trpC2} \Delta y \operatorname{trA}:: e r m C \Delta y \operatorname{tr} E$ & LFH $\rightarrow$ GP3191 \\
\hline GP3197 & 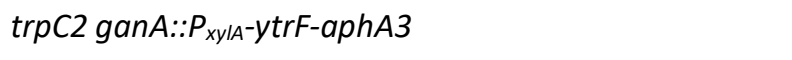 & pGP2184 $\rightarrow 168$ \\
\hline GP3200 & $\operatorname{trpC2}$ amyE::P comG -lacZ-gfp-cat ytrGABCDEF::ermC & GP2646 $\rightarrow$ GP2630 \\
\hline GP3205 & $\operatorname{trp} C 2 \Delta y \operatorname{trCD}:: c a t$ & $\mathrm{LFH} \rightarrow 168$ \\
\hline GP3206 & $\operatorname{trpC2} \Delta y \operatorname{trA}:: e r m C \Delta y \operatorname{tr} B \Delta y \operatorname{tr} E$ & $\mathrm{LFH} \rightarrow \mathrm{GP3} 188$ \\
\hline GP3207 & coml $^{Q 12 L} \triangle y \operatorname{trGABCDEF::ermC}$ & GP2646 $\rightarrow$ DK1042 \\
\hline GP3210 & $\operatorname{trpC2} \Delta r n y:: s p c$ rpoE (Insertion A88) & $\begin{array}{l}\text { Evolution of GP2501 on LB } \\
\text { agar at } 22^{\circ} \mathrm{C}\end{array}$ \\
\hline GP3211d & $\operatorname{trpC2} \Delta r n y:: s p c(r r n W-r r n l)_{2}$ & Evolution of GP2501 at $37^{\circ} \mathrm{C}$ \\
\hline GP3212 & $\operatorname{coml}^{012 L} \Delta y \operatorname{trA}:: s p c$ & GP2641 $\rightarrow$ DK1042 \\
\hline GP3213 & $\operatorname{trp} C 2 \Delta y \operatorname{trA}:: s p c \Delta y \operatorname{trCD}: c a t$ & GP2641 $\rightarrow$ GP3205 \\
\hline GP3216 & $\operatorname{trpC2} \Delta r p o E:: a p h A 3$ & LK1098 $\rightarrow 168$ \\
\hline GP3217 & $\operatorname{trpC2} \Delta r p o E:: a p h A 3 \Delta r n y:: s p c$ & GP2501 $\rightarrow$ GP3216 \\
\hline
\end{tabular}


E. coli

BL21

F ompT gal dcm lon $h s d S_{B}\left(r_{B}^{-} m_{B}^{-}\right) \lambda(D E 3)$

Sambrook et al., 1989

$\operatorname{pLysS}\left(\mathrm{cm}^{R}\right)$

$\mathrm{DH} 5 \alpha$

F- endA1 gInV44 thi-1 recA1 relA1 gyrA96

Sambrook et al., 1989

deoR nupG Ф80dlacZAM15 $\Delta$ (lacZYA-argF)U169,

$h s d R 17\left(r_{K}^{-} m_{K}^{+}\right), \lambda-$

\footnotetext{
a Arrows indicate construction by transformation.

b This genome-reduced strain (see Reuß et al., 2017 for details) was used to amplify the $P_{m t / A}$-ComKS cassette.

c For strains with suppressing point mutations the mutations are indicated using the one- and three letter code for nucleotide and amino acid substitutions, respectively.

d These strains were analyzed by whole genome sequencing.
} 
Table S4: Plasmids used in this study

\begin{tabular}{|c|c|c|c|}
\hline Plasmid & Relevant characteristics & Primers & Reference \\
\hline pDR244 & cre + Ts origin & - & Koo et al., 2017 \\
\hline pGEM-cat & Amplification of the cat cassette & - & Youngman, 1990 \\
\hline pDG646 & Amplification of the ermC cassette & - & Guérout-Fleury et al., 1995 \\
\hline pDG780 & Amplification of the aphA3 cassette & - & Guérout-Fleury et al., 1995 \\
\hline pDG1726 & Amplification of the $s p c$ cassette & - & Guérout-Fleury et al., 1995 \\
\hline pGP888 & $\operatorname{gan} A:: \mathrm{P}_{x y \mid A} ;$ aphA3 & - & Diethmaier et al., 2011 \\
\hline pGP2184 & pGP888-ytrF & MB186/MB187 & This study \\
\hline $\mathrm{pCD} 2$ & Overexpression of $B$. subtilis $\sigma^{A}$ & - & Chang and Doi, 1990 \\
\hline pJOE8999 & CRISPR-Cas9 vector & - & Altenbuchner, 2016 \\
\hline pBSURNAP & $\begin{array}{l}P_{T 7} \text { rpoA rpoZ rpoE rpoY rpoB-rpoC- } \\
8 \times \text { His }\end{array}$ & - & $\begin{array}{l}\text { See Experimental procedures of } \\
\text { Chapter } 2\end{array}$ \\
\hline pGP1331 & Construction of triple FLAG-tag & - & Lehnik-Habrink et al., 2010 \\
\hline pGP2181 & $\begin{array}{l}P_{T 7} \text { rpoA rpoZ rpoE rpoY rpoB-rpoC*- } \\
8 \times \text { His (RpoC-R88H) }\end{array}$ & MB167/MB168 & This study \\
\hline pGP2182 & 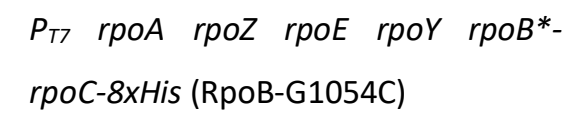 & MB169/MB170 & This study \\
\hline pGP2542 & pGP1331/ greA-3xflag spc & KG412/KG413 & This study \\
\hline pGP2825 & pJOE8999/ rpoC (G263A) & See Table S3 & This study \\
\hline pGP2826 & pJOE8999/rea1 (insertion T33) & See Table S3 & This study \\
\hline pRLG770 & promoter vector & - & Ross et al., 1990 \\
\hline pRLG7558 & $\begin{array}{l}\text { pRLG770 with B. subtilis } \mathrm{P}_{\text {veg }}(-38 /-1 \text {, } \\
+1 G)\end{array}$ & - & Krásný and Gourse, 2004 \\
\hline pRLG7596 & $\begin{array}{l}\text { pRLG770 with } B \text {. subtilis rrnB P1 (- } \\
39 /+1)\end{array}$ & - & Krásný and Gourse, 2004 \\
\hline pLK502 & $\begin{array}{l}\text { pRLG770 with B. subtilis } P_{i / v B}(-262 /-1 \text {, } \\
+1 G G)\end{array}$ & LK\#125/ LK\#127 & This study \\
\hline
\end{tabular}


Table S5: Oligonucleotides used in this study.

\begin{tabular}{|c|c|c|}
\hline Primer & Sequence $^{\mathrm{a}}$ & Purpose \\
\hline MB30 & CTGTATGTCTTTGACCCCTAACTTTTC & $\begin{array}{l}\text { fwd; Detection of ctsR-pdaB region } \\
\text { duplication }\end{array}$ \\
\hline MB208 & СCTCTTTCGCTTGTAAATCTGGT & $\begin{array}{l}\text { rev; Detection of } c t s R-p d a B \text { region } \\
\text { duplication }\end{array}$ \\
\hline NC9 & CTATGAAAAGATGTTTACGCCAGGG & fwd; Control of rny deletion \\
\hline ML101 & CTGCAAATTAATGACTGCTAGTTCTT & rev; Control of rny deletion \\
\hline LK\#2684 & $\begin{array}{l}\text { GGTCTAGAGCGGCCGCTTTAAGAAGGAGATATAT } \\
\text { CTATGACAGGTCAACTAGTTC }\end{array}$ & Construction of pBSURNAP \\
\hline LK\#2685 & $\begin{array}{l}\text { CGCGGATCCGGTACCCCATGGCGCGCAAGTTCTTT } \\
\text { TGTTACTACATCG }\end{array}$ & Construction of pBSURNAP \\
\hline LK\#2686 & $\begin{array}{l}\text { GCGCCATGGTGGCTCGGGTGCAATGCTAGATGTG } \\
\text { AACAATTTTGAG }\end{array}$ & Construction of pBSURNAP \\
\hline LK\#2687 & $\begin{array}{l}\text { GCGGTACCTTAGTGATGGTGATGGTGATGGTGAT } \\
\text { GTTCAACCGGGACCATATCG }\end{array}$ & Construction of pBSURNAP \\
\hline MB167 & $\begin{array}{l}\text { AAACCATGGTGGCTCGGGTGCAATGCTAGATGTG } \\
\text { AACAATTTTGAGTATATGAAC }\end{array}$ & $\begin{array}{l}\text { fwd; Amplification of } r p o C \text { from GP2912 for } \\
\text { cloning into pBSURNAP, } \underline{\mathrm{Ncol}}\end{array}$ \\
\hline MB168 & $\begin{array}{l}\text { AAAGGTACCCTAGTGATGGTGATGGTGATGGTGA } \\
\text { TGTTCAACCGGGACCATATCGT }\end{array}$ & $\begin{array}{l}\text { fwd; Amplification of } r p o C \text { for cloning into } \\
\text { pBSURNAP, } \underline{\text { Kpnl }}\end{array}$ \\
\hline MB169 & $\begin{array}{l}\text { AAAGCGGCCGCTTTAAGAAGGAGATATATCTATGA } \\
\text { CAGGTCAACTAGTTCAGTATGGAC }\end{array}$ & $\begin{array}{l}\text { fwd; Amplification of } r p o B \text { from GP2913 for } \\
\text { cloning into pBSURNAP, №tl }\end{array}$ \\
\hline MB170 & $\begin{array}{l}\text { AAACCATGGCGCGCAAGTTCTTTTGTTACTACATC } \\
\text { GCGTTCAA }\end{array}$ & $\begin{array}{l}\text { fwd; Amplification of } r p o B \text { from GP2913 for } \\
\text { cloning into pBSURNAP, } \underline{\mathrm{Ncol}}\end{array}$ \\
\hline MB17 & CGCCGAACTGGAAGAGTCATTCC & $\begin{array}{l}\text { rev; Amplification of upstream fragment } \\
\text { (deletion of } \operatorname{csp} D \text { ) }\end{array}$ \\
\hline MB18 & $\begin{array}{l}\text { CCTATCACCTCAAATGGTTCGCTGGTTGAACCATTT } \\
\text { TACTTTACCGTTTTGCAT }\end{array}$ & $\begin{array}{l}\text { fwd; Amplification of upstream fragment } \\
\text { (deletion of } c s p D \text { ) }\end{array}$ \\
\hline MB19 & $\begin{array}{l}\text { CCGAGCGCCTACGAGGAATTTGTATCGGTAATCGT } \\
\text { GGACCTCAAGCTTCTAATGTTG }\end{array}$ & $\begin{array}{l}\text { fwd; Amplification of downstream } \\
\text { fragment (deletion of } c s p D \text { ) }\end{array}$ \\
\hline MB20 & GAAGCACTCCTTGAATCGCTGAAGC & $\begin{array}{l}\text { rev; Amplification of downstream fragment } \\
\text { (deletion of } c s p D \text { ) }\end{array}$ \\
\hline MB21 & GGCGAACTTGTCGATGAACATCAG & fwd; Sequencing $\operatorname{csp} D$ deletion \\
\hline MB22 & GGCAGCTGGCCTTGTTATGATC & rev; Sequencing $\operatorname{csp} D$ deletion \\
\hline VK17 & GACGAAGACGGAAATGAGCTAGATGC & $\begin{array}{l}\text { fwd; Amplification of upstream fragment } \\
\text { (deletion of greA) }\end{array}$ \\
\hline VK18 & $\begin{array}{l}\text { СCTATCACCTCAAATGGTTCGCTGGTTCAAGTTTTT } \\
\text { GTTTTCCTTCTGCAGTCATAGG }\end{array}$ & $\begin{array}{l}\text { rev; Amplification of upstream fragment } \\
\text { (deletion of greA) }\end{array}$ \\
\hline VK19 & $\begin{array}{l}\text { CGAGCGCCTACGAGGAATTTGTATCGGATGAAGA } \\
\text { AGTCACAGTACAAACACCGG }\end{array}$ & $\begin{array}{l}\text { fwd; Amplification of downstream } \\
\text { fragment (deletion of greA) }\end{array}$ \\
\hline VK20 & TGCAGCTGCGGCAATGACTGTTTTAAAAAC & $\begin{array}{l}\text { rev; Amplification of downstream fragment } \\
\text { (deletion of greA) }\end{array}$ \\
\hline
\end{tabular}




\begin{tabular}{|c|c|c|}
\hline VK21 & GGCTTAGTGCTGAATTATGATGAAGATACAC & fwd; Sequencing greA deletion \\
\hline VK22 & GTGCCTTTGTCGTCCCCCGG & rev; Sequencing greA deletion \\
\hline ML47 & 5'-GAAGAATCTGCTTACACATACATCG & $\begin{array}{l}\text { fwd; Amplification of upstream fragment } \\
\text { (deletion of } r n y \text { ) }\end{array}$ \\
\hline KG409 & $\begin{array}{l}\text { GACTGTGTTTTATATTTTTCTCGTTCATACTTTCACC } \\
\text { TCCTCTTGCTATGAACT }\end{array}$ & $\begin{array}{l}\text { rev; Amplification of upstream fragment } \\
\text { (deletion of } r n y \text { ) }\end{array}$ \\
\hline KG410 & $\begin{array}{l}\text { CCGAGCGCCTACGAGGAATTTGTATCGAGTGATGC } \\
\text { GCTAAGCATCACTTTATTTTTTG }\end{array}$ & $\begin{array}{l}\text { fwd; Amplification of downstream } \\
\text { fragment (deletion of } r n y)\end{array}$ \\
\hline NP60 & $\begin{array}{l}\text { GCAGACACATACTCTCCCACTTTTACACTGCTGACA } \\
\mathrm{T}\end{array}$ & $\begin{array}{l}\text { rev; Amplification of downstream fragment } \\
\text { (deletion of } r n y \text { ) }\end{array}$ \\
\hline KG411 & ATGAACGAGAAAAATATAAAACACAGTC & $\begin{array}{l}\text { fwd; Amplification of ermC cassette } \\
\text { (deletion of } r n y \text { ) }\end{array}$ \\
\hline CZ68 & $\begin{array}{l}\text { CGATACAAATTCCTCGTAGGCGCTCGGTTACTTATT } \\
\text { AAATAATTTATAGCTATTG }\end{array}$ & $\begin{array}{l}\text { rev; Amplification of ermC cassette } \\
\text { (deletion of } r n y \text { ) }\end{array}$ \\
\hline KG414 & GTCGGTTCATCACAAAAAGCGCTGAT & fwd; Sequencing rny deletion \\
\hline NP61 & AGTATTGGTACACACATGAGATTTTCCTGTTAG & rev; Sequencing rny deletion \\
\hline NC16 & CTGCCACTGAATTTGGACTCG & rev; Sequencing rny deletion \\
\hline JN420 & $\begin{array}{l}\text { CCTATCACCTCAAATGGTTCGCTGCGCACATGTCTA } \\
\text { TGTAAGATAATCGT }\end{array}$ & $\begin{array}{l}\text { rev; Amplification of upstream fragment } \\
\text { (deletion of } n r n A \text { ) }\end{array}$ \\
\hline JN421 & GGGATCGAAGTGCTTCCCG & $\begin{array}{l}\text { fwd; Amplification of upstream fragment } \\
\text { (deletion of } n r n A \text { ) }\end{array}$ \\
\hline JN422 & $\begin{array}{l}\text { CCGAGCGCCTACGAGGAATTTGTATCGGCTGGGAT } \\
\text { GAAGCTGATCGTA }\end{array}$ & $\begin{array}{l}\text { fwd; Amplification of downstream } \\
\text { fragment (deletion of } n r n A \text { ) }\end{array}$ \\
\hline JN423 & GCGGCATACTCGAAGGCA & $\begin{array}{l}\text { rev; Amplification of downstream fragment } \\
\text { (deletion of } n r n A \text { ) }\end{array}$ \\
\hline JN424 & GACCAAAAATCCCGTCACGG & fwd; Sequencing $n r n A$ deletion \\
\hline JN425 & GCTTGCCAACCGGTTAAAAATATG & rev; Sequencing $n r n A$ deletion \\
\hline MB31 & CTGCGTATATCTGCTTCGAAATCCTTC & $\begin{array}{l}\text { fwd; Amplification of upstream fragment } \\
\text { (integration of } \mathrm{P}_{m t / A} \text {-comKS) }\end{array}$ \\
\hline MB32 & $\begin{array}{l}\text { TAAAAATAAAAAGCTAGCGGGGATCCCAAGTCAA } \\
\text { AACCGAGTCTCATTTCCTATTTATCC }\end{array}$ & $\begin{array}{l}\text { rev; Amplification of upstream fragment } \\
\text { (integration of } \mathrm{P}_{m t / A} \text {-comKS) }\end{array}$ \\
\hline MB33 & CTTGGGATCCCCGCTAGCTTTTTATTTTTA & $\begin{array}{l}\text { fwd; Amplification of } \mathrm{P}_{m t / A} \text {-comKS for its } \\
\text { insertion into yvcA-hisl locus }\end{array}$ \\
\hline MB34 & $\begin{array}{l}\text { CCTATCACCTCAAATGGTTCGCTGCGGAGGATTTC } \\
\text { GTGCCGGTTGATTA }\end{array}$ & $\begin{array}{l}\text { rev; Amplification of } \mathrm{P}_{m t \mid A} \text {-comKS for its } \\
\text { insertion into } y v c A-h i s / \text { locus }\end{array}$ \\
\hline MB35 & $\begin{array}{l}\text { CCGAGCGCCTACGAGGAATTTGTATCG } \\
\text { GCCGGCTAGCACCCAATATAAATCTAAAT }\end{array}$ & $\begin{array}{l}\text { fwd; Amplification of downstream } \\
\text { fragment (integration of } \mathrm{P}_{m t / A} \text {-comKS) }\end{array}$ \\
\hline MB36 & GTGCTGACACTTGCGTATATGAACAAG & $\begin{array}{l}\text { rev; Amplification of downstream fragment } \\
\text { (integration of } \mathrm{P}_{m t / A}-\text { comKS) }\end{array}$ \\
\hline MB37 & GTAAACTCCTTTGTAGCCTCATACTGAC & fwd; Sequencing $P_{m t / A}$-comKS insertion \\
\hline
\end{tabular}




\begin{tabular}{|c|c|c|}
\hline MB38 & GAATGTGAGATGAAACAGGCAGATGAAC & rev; Sequencing $\mathrm{P}_{m t / A}$-comKS insertion \\
\hline MB43 & CTTGATAGATACTTTCCATCCTCCGG & fwd; Sequencing $\mathrm{P}_{m t / A}$-comKS insertion \\
\hline MB44 & СССТАСАСТTTCTTCGACAAGACCC & fwd; Sequencing $\mathrm{P}_{m t / A}-$ comKS insertion \\
\hline MB60 & GCTGATGAAACGGCAGTGCT & $\begin{array}{l}\text { fwd; Amplification of upstream fragment } \\
\text { (deletion of } f t s H \text { ) }\end{array}$ \\
\hline MB61 & $\begin{array}{l}\text { CCTATCACCTCAAATGGTTCGCTGTCCTTACСTССТC } \\
\text { CCACAG }\end{array}$ & $\begin{array}{l}\text { rev; Amplification of upstream fragment } \\
\text { (deletion of } f t s H \text { ) }\end{array}$ \\
\hline MB62 & $\begin{array}{l}\text { CCGAGCGCCTACGAGGAATTTGTATCGAAGACGAT } \\
\text { ACGAAAGAGTAATTCGC }\end{array}$ & $\begin{array}{l}\text { fwd; Amplification of downstream } \\
\text { fragment (deletion of } f t s H \text { ) }\end{array}$ \\
\hline MB63 & СТССТАTACACTTCCTACGCGG & $\begin{array}{l}\text { rev; Amplification of downstream fragment } \\
\text { (deletion of } f t s H \text { ) }\end{array}$ \\
\hline MB64 & GGGCTGAAGGTGGTCAAATC & fwd; Sequencing $f t s H$ deletion \\
\hline MB65 & CATATCAGTCGTTCTCGCTGCA & rev; Sequencing $\mathrm{ftsH}$ deletion \\
\hline MB66 & CATCGGTCCGGTTTCCAGCA & $\begin{array}{l}\text { fwd; Amplification of upstream fragment } \\
\text { (deletion of } y \text { trA) }\end{array}$ \\
\hline MB67 & $\begin{array}{l}\text { CCTATCACCTCAAATGGTTCGCTGGGGTGTTGAGC } \\
\text { TTCTTGGATC }\end{array}$ & $\begin{array}{l}\text { rev; Amplification of upstream fragment } \\
\text { (deletion of } y t r A \text { ) }\end{array}$ \\
\hline MB68 & $\begin{array}{l}\text { CCGAGCGCCTACGAGGAATTTGTATCGGCTGATGT } \\
\text { GAAGGGAGGCAA }\end{array}$ & $\begin{array}{l}\text { fwd; Amplification of downstream } \\
\text { fragment (deletion of } y t r A \text { ) }\end{array}$ \\
\hline MB69 & GGCGATCAAGACACCCTTGA & $\begin{array}{l}\text { rev; Amplification of downstream fragment } \\
\text { (deletion of } y \operatorname{trA} \text { ) }\end{array}$ \\
\hline MB70 & GATGTACTTGCCGTCCTTCCA & fwd; Sequencing ytrA deletion \\
\hline MB71 & ACCCGGCACCCAGTTGATAT & rev; Sequencing ytrA deletion \\
\hline MB72 & AGGGGACAGAGTATCTCAGGCA & $\begin{array}{l}\text { fwd; Amplification of upstream fragment } \\
\text { (deletion of comEC) }\end{array}$ \\
\hline MB73 & $\begin{array}{l}\text { CCTATCACCTCAAATGGTTCGCTGCGCATTCATCAC } \\
\text { ACGTAGCTC }\end{array}$ & $\begin{array}{l}\text { rev; Amplification of upstream fragment } \\
\text { (deletion of comEC) }\end{array}$ \\
\hline MB74 & $\begin{array}{l}\text { CCGAGCGCCTACGAGGAATTTGTATCGAAAGACTG } \\
\text { CCGAGAAATCAGCA }\end{array}$ & $\begin{array}{l}\text { fwd; Amplification of downstream } \\
\text { fragment (deletion of comEC) }\end{array}$ \\
\hline MB75 & TCTCCAATAAACGTGCAGAGCTT & $\begin{array}{l}\text { rev; Amplification of downstream fragment } \\
\text { (deletion of comEC) }\end{array}$ \\
\hline MB76 & AACAACGACGAGTCAAACGAAACAA & fwd; Sequencing comEC deletion \\
\hline MB77 & CTCTGTTCGTTTTCGGTTGACG & rev; Sequencing comEC deletion \\
\hline MB78 & AACCGTTTATCCGAGGTCAGCC & $\begin{array}{l}\text { fwd; Amplification of upstream fragment } \\
\text { (deletion of } d e g U \text { ) }\end{array}$ \\
\hline MB79 & $\begin{array}{l}\text { CCTATCACCTCAAATGGTTCGCTGGTTTACTTTAGT } \\
\text { CACAAGCCACGC }\end{array}$ & $\begin{array}{l}\text { rev; Amplification of upstream fragment } \\
\text { (deletion of } \operatorname{deg} U \text { ) }\end{array}$ \\
\hline MB80 & $\begin{array}{l}\text { CCGAGCGCCTACGAGGAATTTGTATCGGGCTGGGT } \\
\text { AGAAATGAGATAGTA }\end{array}$ & $\begin{array}{l}\text { fwd; Amplification of downstream } \\
\text { fragment (deletion of } \operatorname{deg} U \text { ) }\end{array}$ \\
\hline
\end{tabular}




\begin{tabular}{|c|c|c|}
\hline MB81 & AGCACGCCTCCTTTCGAAACAG & $\begin{array}{l}\text { rev; Amplification of downstream fragment } \\
\text { (deletion of } d e g U \text { ) }\end{array}$ \\
\hline MB82 & GCAGGTGTATGAAGTGATTGAGC & fwd; Sequencing degU deletion \\
\hline MB83 & TCGAAGCGTCTGCTGCAATTC & rev; Sequencing degU deletion \\
\hline MB70 & GATGTACTTGCCGTCCTTCCA & $\begin{array}{l}\text { fwd; Amplification of upstream fragment } \\
\text { (deletion of ytr operon) }\end{array}$ \\
\hline MB118 & $\begin{array}{l}\text { CCTATCACCTCAAATGGTTCGCTGCACTTAATACAA } \\
\text { TAAATACTTTGACTCACA }\end{array}$ & $\begin{array}{l}\text { rev; Amplification of upstream fragment } \\
\text { (deletion of ytr operon) }\end{array}$ \\
\hline MB119 & $\begin{array}{l}\text { CCGAGCGCCTACGAGGAATTTGTATCGTATAATGC } \\
\text { GAACGAGCCGGC }\end{array}$ & $\begin{array}{l}\text { fwd; Amplification of downstream } \\
\text { fragment (deletion of ytr operon) }\end{array}$ \\
\hline MB120 & GCACAAATACACCATATAAAGTACATTCC & $\begin{array}{l}\text { rev; Amplification of downstream fragment } \\
\text { (deletion of ytr operon) }\end{array}$ \\
\hline MB121 & CGATCGAAATGCCGACCAC & fwd; Sequencing ytr operon deletion \\
\hline MB122 & GTTCATTTATGGCTGTCACATCGAG & rev; Sequencing ytr operon deletion \\
\hline MB70 & GATGTACTTGCCGTCCTTCCA & $\begin{array}{l}\text { fwd; Amplification of upstream fragment } \\
\text { (deletion of } y \operatorname{trG}-E \text { region) }\end{array}$ \\
\hline MB118 & $\begin{array}{l}\text { CCTATCACCTCAAATGGTTCGCTGCACTTAATACAA } \\
\text { TAAATACTTTGACTCACA }\end{array}$ & $\begin{array}{l}\text { rev; Amplification of upstream fragment } \\
\text { (deletion of } y \operatorname{trG}-E \text { region) }\end{array}$ \\
\hline MB194 & $\begin{array}{l}\text { CCGAGCGCCTACGAGGAATTTGTATCGTTGAGGTT } \\
\text { TAAGGATCAGGTTCATTTTAT }\end{array}$ & $\begin{array}{l}\text { fwd; Amplification of downstream } \\
\text { fragment (deletion of } y \operatorname{trG}-E \text { region) }\end{array}$ \\
\hline MB195 & GATACATCCGACAAAGATCAGTCC & $\begin{array}{l}\text { rev; Amplification of downstream fragment } \\
\text { (deletion of } y \operatorname{trG}-E \text { region) }\end{array}$ \\
\hline MB121 & CGATCGAAATGCCGACCAC & fwd; Sequencing ytrG-E region deletion \\
\hline MB187 & TTATAATTCTCTTCTCAACGCTGTCAG & rev; Sequencing ytrG-E region deletion \\
\hline MB68 & $\begin{array}{l}\text { CCGAGCGCCTACGAGGAATTTGTATCGGCTGATGT } \\
\text { GAAGGGAGGCAA }\end{array}$ & fwd; Confirmation of ytrC deletion \\
\hline MB180 & GACACAGCCTTGATAGATGAGATAC & rev; Confirmation of $y \operatorname{trC}$ deletion \\
\hline CB449 & $\begin{array}{l}\text { CCTATCACCTCAAATGGTTCGCTGTCCTTAAACCTC } \\
\text { AACGGTAATTCCT }\end{array}$ & fwd; Confirmation of $y \operatorname{trD}$ deletion \\
\hline MB179 & CTGGATTCTTTGTGAGCTACTTCTC & rev; Confirmation of $y \operatorname{tr} D$ deletion \\
\hline CB448 & TCACCATATTATTTAGTCATTCCGGC & fwd; Confirmation of ytrE deletion \\
\hline CB449 & $\begin{array}{l}\text { CCTATCACCTCAAATGGTTCGCTGTCCTTAAACCTC } \\
\text { AACGGTAATTCCT }\end{array}$ & rev; Confirmation of $y \operatorname{trE}$ deletion \\
\hline MB121 & CGATCGAAATGCCGACCAC & $\begin{array}{l}\text { Fwd; Amplification of ytrAB:ermC from } \\
\text { GP3193 }\end{array}$ \\
\hline MB180 & GACACAGCCTTGATAGATGAGATAC & $\begin{array}{l}\text { rev; Amplification of ytrAB:ermC from } \\
\text { GP3193 }\end{array}$ \\
\hline MB186 & $\begin{array}{l}\text { TTTTCTAGATATGAGGTTTAAGGATCAGGTTCATTT } \\
\text { TAT }\end{array}$ & $\begin{array}{l}\text { fwd; Amplification of } y \operatorname{tr} F \text { for cloning into } \\
\text { pGP888, } \underline{\text { bal }}\end{array}$ \\
\hline
\end{tabular}




\begin{tabular}{|c|c|}
\hline MB187 & ATTGGTACCTTATAATTCTCTTCTCAACGCTGTCAG \\
\hline MB198 & GCGGCAGCTGTCAAAAGC \\
\hline MB199 & $\begin{array}{l}\text { CСTATCACCTCAAATGGTTCGCTGCTACCATCTCCG } \\
\text { СТTСССТC }\end{array}$ \\
\hline MB200 & $\begin{array}{l}\text { CCGAGCGCCTACGAGGAATTTGTATCGGTAAGGG } \\
\text { AGAGAGAACATATGATTG }\end{array}$ \\
\hline MB201 & CTCCTTCCTTGCCCATTACG \\
\hline MB202 & CACTATGCAGGGGTTGAGCT \\
\hline MB203 & GTTTGGTTCATACACTTGCGTTC \\
\hline CB448 & TCACCATATTATTTAGTCATTCCGGC \\
\hline CB449 & $\begin{array}{l}\text { CCTATCACCTCAAATGGTTCGCTGTCCTTAAACCTC } \\
\text { AACGGTAATTCCT }\end{array}$ \\
\hline CB450 & $\begin{array}{l}\text { CGAGCGCCTACGAGGAATTTGTATCGTTATAATGC } \\
\text { GAACGAGCCGGCT }\end{array}$ \\
\hline CB451 & TCCCATGTTTTCAAGCTTTTATAAAACG \\
\hline CB452 & ACCTCGAGATCCTTTTTTGGCG \\
\hline CB453 & TGCTAAGCGATGCCGTGCT \\
\hline SW17 & GACATTGTCCCTTTATCAGC \\
\hline SW18 & $\begin{array}{l}\text { GGGGTGTGAGCTGAATTCCTGCTGTCTGATCAATT } \\
\text { TAATG }\end{array}$ \\
\hline SW19 & $\begin{array}{l}\text { CCGAGCGCCTACGAGGAATTTGTATCGCCCCCATG } \\
\text { AAAAAAAGAC }\end{array}$ \\
\hline SW20 & CGAATCAAATGCTTATTTGG \\
\hline SW21 & GAATTCAGCTCACACCCC \\
\hline SW40 & $\begin{array}{l}\text { TGGTTTTTCAATCTCGATCATTATTTTCCCTCCTTTT } \\
\text { C }\end{array}$ \\
\hline SW23 & ATGATCGAGATTGAAAAACCA \\
\hline SW24 & $\begin{array}{l}\text { CCTATCACCTCAAATGGTTCGCTGTCAATCGTCTTT } \\
\text { GCGAAG }\end{array}$ \\
\hline SW25 & GATCATAATCTTCAATGCGAAG \\
\hline SW27 & GAACAACCACAAATGACATC \\
\hline SW28 & GTGATCTGTGAAAATCCAAAG \\
\hline
\end{tabular}

rev; Amplification of $y t r F$ for cloning into pGP888, Kpnl

fwd; Amplification of upstream fragment (deletion of $y \operatorname{trCD}$ )

rev; Amplification of upstream fragment (deletion of $y \operatorname{trCD}$ )

fwd; Amplification of downstream fragment (deletion of ytrCD)

rev; Amplification of downstream fragment (deletion of $y \operatorname{trCD}$ )

fwd; Sequencing ytrCD deletion

rev; Sequencing ytrCD deletion

fwd; Amplification of upstream fragment (deletion of ytrF)

rev; Amplification of upstream fragment (deletion of $y \operatorname{trF}$ )

fwd; Amplification of downstream fragment (deletion of $y t r F$ )

rev; Amplification of downstream fragment (deletion of $y$ trF)

fwd; Sequencing ytrF deletion

rev; Sequencing ytrF deletion

fwd; Amplification of upstream fragment (insertion of rpoA)

rev; Amplification of upstream fragment (insertion of rpoA)

fwd; Amplification of downstream fragment (insertion of $r p o A$ )

rev; Amplification of downstream fragment (insertion of rpoA)

fwd; Amplification of $\mathrm{P}_{\text {rps }}$

rev; Amplification of $\mathrm{P}_{\text {rps }}$

fwd; Amplification of $r p o A$

rev; Amplification of rpoA

fwd; Sequencing rpoA insertion

rev; Sequencing rpoA insertion

fwd; Amplification of upstream fragment (deletion of rpoA) 


\begin{tabular}{|c|c|}
\hline SW29 & $\begin{array}{l}\text { CCTATCACCTCAAATGGTTCGCTGTACTTAAAACCC } \\
\text { TCCTTCAAAAC }\end{array}$ \\
\hline SW30 & $\begin{array}{l}\text { ATATTTTACTGGATGAATTGTTTTAGTAACTAGTTT } \\
\text { CCCTTGTGAACTAGG }\end{array}$ \\
\hline SW31 & CAACTCTCTGCTTTTGGC \\
\hline SW32 & GCGATGTTCAAAGTTGAAC \\
\hline SW33 & CATATTTTTTTACCGCCATTCA \\
\hline SW41 & TTGTCAAGTGAAGGCGCGCTAT \\
\hline mls-rev (kan) & $\begin{array}{l}\text { CGATACAAATTCCTCGTAGGCGCTCGGGCCGACTG } \\
\text { CGCAAAAGACATAATCG }\end{array}$ \\
\hline SW42 & GTGAAGGAAAAGGGATG \\
\hline SW43 & $\begin{array}{l}\text { CCGAGCGCCTACGAGGAATTTGTATCGGTTCTGTA } \\
\text { GATTCACTCCGA }\end{array}$ \\
\hline SW44 & $\begin{array}{l}\text { ATAGCGCGCCTTCACTTGACAAGAGACTTAGATTA } \\
\text { AGTTGACGC }\end{array}$ \\
\hline SW45 & ACTGTCAATATAGCATAAATTCC \\
\hline KG412 & $\begin{array}{l}\text { AAAGGATCCATGGCACAAGAGAAAGTTTTTCCTAT } \\
\mathrm{G}\end{array}$ \\
\hline KG413 & $\begin{array}{l}\text { TTTGTCGACTGAAATTTTCACAATTTTCACGAGCAT } \\
\text { TTC }\end{array}$ \\
\hline LK\#125 & GGGAATTCATGGATTGCAAGATGATCTG \\
\hline LK\#127 & CCAAGCTTAGACCGAACTCATATTACGCCGC \\
\hline SW69 & AAGGCCAACGAGGCCCTTACTCACTTGTTAC \\
\hline SW70 & AAGGCCTTATTGGCCTCTTGAAGCATACG \\
\hline SW73 & aAGgATGGGaCACATTGAACTGGCTG \\
\hline SW74 & tCCCATcCTtTCACGAtGGACTTTAGC \\
\hline SW93 & (p)IACGTAAAGTCCGTCGTGAGAGAA \\
\hline SW94 & 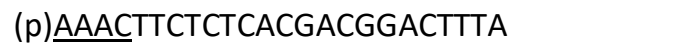 \\
\hline SW81 & AAGGCCAACGAGGCCCAAGCACAGCAAGTGATT \\
\hline SW82 & $\begin{array}{l}\text { aATGTTGTAgCCaTCgACcAACAGGATATCCATGGG } \\
\mathrm{T}\end{array}$ \\
\hline
\end{tabular}

rev; Amplification of upstream fragment (deletion of $r p o A$ )

fwd; Amplification of downstream fragment (deletion of rpoA)

rev; Amplification of downstream fragment (deletion of $r p o A$ )

fwd; Sequencing rpoA deletion

rev; Sequencing rpoA deletion

fwd; Amplification of $\mathrm{P}_{\text {alf4- }}$-gfp-ermC

rev; Amplification of $\mathrm{P}_{\text {alf4-gfp-ermC }}$

fwd; Amplification of upstream fragment (insertion of $\mathrm{P}_{\text {alf4-gfp-ermC) }}$

rev; Amplification of upstream fragment (insertion of $\mathrm{P}_{\text {alf } 4 \text {-gfp-ermC) }}$

fwd; Amplification of downstream fragment (insertion of $\mathrm{P}_{\text {alf4-gfp-ermC) }}$

fwd; Amplification of downstream fragment (insertion of $\mathrm{P}_{\text {alf4-gfp-ermC) }}$

fwd; Amplification of greA for cloning into pGP1331, BamHI

rev; Amplification of greA for cloning into pGP1331, Sall

rev; Amplification of $\mathrm{P}_{\text {ilvB }}$ for cloning into pRLG770, EcoRI

rev; Amplification of $\mathrm{P}_{i l v B}$ for cloning into pRLG770, $\underline{\text { HindIII }}$

fwd; rpoC CRISPR/Cas9 template; Sfil; to create $\mathrm{PGP} 2825$

rev; rpoC CRISPR/Cas9 template; Sfil; to create PGP2825

fwd; rpoC CRISPR/Cas9 mutagenesis; to create $\mathrm{PGP} 2825$

rev; rpoC CRISPR/Cas9 mutagenesis; to create PGP2825

fwd; rpoC CRISPR/Cas9 target sequnce; Bsal; to create pGP2825

rev; rpoC CRISPR/Cas9 target sequnce; Bsal; to create pGP2825

fwd; rae1 CRISPR/Cas9 template; Sfil; to create PGP2826

rev; rae1 CRISPR/Cas9 mutagenesis; to create pGP2826 


\begin{tabular}{|c|c|}
\hline SW83 & gGTcGAtGGcTACAACATtGATTGGAGCC \\
\hline SW84 & AAGGCCTTATTGGCCCCTGAACAATATCCTCTCTG \\
\hline SW85 & (p)IACGTGGATATCCTGTTAGTAGAC \\
\hline SW86 & 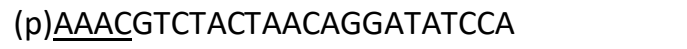 \\
\hline KG227 & GAAGGAATCAGAAATGATGACCGCCA \\
\hline KG228 & CGCTGTTTCCACCGCTAGTTCCA \\
\hline MB5 & CACGCAAATCTATGAAGGCACTC \\
\hline MB6 & GCTACAATACCCTTTCCAAGTGAG \\
\hline MB206 & GTCTTGCCTCCGATGACTTTC \\
\hline MB207 & GCGCCTGTTTCAACCAGCA \\
\hline KG384 & GAACGAGGACTGCCCTGTGTTCTC \\
\hline KG385 & CTGCCAGCTTCATTCGTTTCGATATCTTC \\
\hline MB9 & GAAGGCGTATCTGAGCGTGACG \\
\hline MB176 & GAATCGCCTCTTCAATCAGAGAC \\
\hline MB177 & TGGATGTATCGCCTAAGCAGGTT \\
\hline SW63 & GATTCTTCCTGAAGAGGATATG \\
\hline KG422 & GGATCAGTTACAACGTAAGAAGC \\
\hline MB108 & GCGCTCAATTGTTTCAGTTCCTTC \\
\hline MB175 & CAATTGTCCCGCAGTATAAGCTG \\
\hline SW77 & GATACCGCTCTTAAAACTGC \\
\hline SW87 & GAAACAAGCCTTCTTGGA \\
\hline SW88 & CGTACCATCACGTATGAAC \\
\hline SW79 & GAATACCGGTTGCATCTG \\
\hline SW80 & AGATTATTGAGCAAATCAGTG \\
\hline
\end{tabular}

fwd; rae 1 CRISPR/Cas9 mutagenesis; to create $\mathrm{PGP} 2826$

rev; rae1 CRISPR/Cas9 template; Sfil; to create PGP2826

fwd; rae1 CRISPR/Cas9 target sequnce; Bsal; to create pGP2826

rev; rae1 CRISPR/Cas9 target sequnce; Bsal; to create pGP2826

fwd; Sequencing $\operatorname{csp} D$

rev; Sequencing $\operatorname{csp} D$

fwd; Sequencing rpoE

rev; Sequencing rpoE

fwd; Sequencing adeR

rev; Sequencing adeR

fwd; Sequencing greA

rev; Sequencing greA

fwd; Sequencing rpoB

fwd; Sequencing rpoB

fwd; Sequencing rpoB

fwd; Sequencing rpoB

rev; Sequencing rpoB

rev; Sequencing rpoC

rev; Sequencing rpoC

fwd; Sequencing rpoC

fwd; Sequencing rpoC

fwd; Sequencing rpoC

fwd; Sequencing of pGP2825/pGP2826

fwd; Sequencing of pGP2825/pGP2826 


\begin{tabular}{|c|c|c|}
\hline M13fwd & GTAAAACGACGGCCAGTG & fwd; Sequencing of pGP2542 \\
\hline cat-fwd (kan) & $\begin{array}{l}\text { CAGCGAACCATTTGAGGTGATAGGCGGCAATAGT } \\
\text { TACCCTTATTATCAAG }\end{array}$ & $\begin{array}{l}\text { fwd; Amplification of chloramphenicol } \\
\text { resistance cassette }\end{array}$ \\
\hline cat-rev (kan) & $\begin{array}{l}\text { CGATACAAATTCCTCGTAGGCGCTCGGCCAGCGTG } \\
\text { GACCGGCGAGGCTAGTTACCC }\end{array}$ & $\begin{array}{l}\text { rev; Amplification of chloramphenicol } \\
\text { resistance cassette }\end{array}$ \\
\hline $\begin{array}{l}\text { cat-rev w/o T. } \\
\text { (kan) }\end{array}$ & $\begin{array}{l}\text { CGATACAAATTCCTCGTAGGCGCTCGGTTATAAAA } \\
\text { GCCAGTCATTAGGCCTATC }\end{array}$ & $\begin{array}{l}\text { rev; Amplification of chloramphenicol } \\
\text { resistance cassette without Term. }\end{array}$ \\
\hline kan-fwd & CAGCGAACCATTTGAGGTGATAGG & $\begin{array}{l}\text { fwd; Amplification of kanamycin resistance } \\
\text { cassette }\end{array}$ \\
\hline kan-rev & CGATACAAATTCCTCGTAGGCGCTCGG & $\begin{array}{l}\text { rev; Amplification of kanamycin resistance } \\
\text { cassette }\end{array}$ \\
\hline $\begin{array}{l}\text { kan-rev w/o } \\
\text { T. }\end{array}$ & TTACTAAAACAATTCATCCAGTAAAATAT & $\begin{array}{l}\text { rev; Amplification of kanamycin resistance } \\
\text { cassette without Term. }\end{array}$ \\
\hline mls-fwd (kan) & $\begin{array}{l}\text { CAGCGAACCATTTGAGGTGATAGGGATCCTTTAAC } \\
\text { TCTGGCAACCCTC }\end{array}$ & $\begin{array}{l}\text { fwd; Amplification of erythromycin } \\
\text { resistance cassette }\end{array}$ \\
\hline mls-rev (kan) & $\begin{array}{l}\text { CGATACAAATTCCTCGTAGGCGCTCGGGCCGACTG } \\
\text { CGCAAAAGACATAATCG }\end{array}$ & $\begin{array}{l}\text { rev; Amplification of erythromycin } \\
\text { resistance cassette }\end{array}$ \\
\hline $\begin{array}{l}\text { mls-rev w/o } \\
\text { T. (kan) }\end{array}$ & $\begin{array}{l}\text { CGATACAAATTCCTCGTAGGCGCTCGGTTACTTATT } \\
\text { AAATAATTTATAGCTATTG }\end{array}$ & $\begin{array}{l}\text { rev; Amplification of erythromycin } \\
\text { resistance cassette without Term. }\end{array}$ \\
\hline spc-fwd (kan) & $\begin{array}{l}\text { CAGCGAACCATTTGAGGTGATAGGGACTGGCTCG } \\
\text { CTAATAACGTAACGTGACTGGCAAGAG }\end{array}$ & $\begin{array}{l}\text { fwd; Amplification of spectinomycin } \\
\text { resistance cassette }\end{array}$ \\
\hline $\begin{array}{l}\text { spc-rev w/o } \\
\text { T. (kan) }\end{array}$ & $\begin{array}{l}\text { CGATACAAATTCCTCGTAGGCGCTCGGGTAGTATT } \\
\text { TTTTGAGAAGATCAC }\end{array}$ & $\begin{array}{l}\text { rev; Amplification of spectinomycin } \\
\text { resistance cassette without Term. }\end{array}$ \\
\hline
\end{tabular}

a Homologous bases for joining PCR are shown in italics, restriction sites are underlined, phosphorylated primers are indicated with (p). Mutation positions are written in lower case. 


\section{Curriculum Vitae}

\section{Personal information}

Name: $\quad$ Martin Benda

Date of birth: $\quad 5^{\text {th }}$ April 1992

Place of birth: $\quad$ Prague, Czech Republic

Nationality: $\quad$ Czech

Contact: $\quad$ mb.martin.benda[at]gmail.com

\section{Education}

- 2017 - 2020: PhD studies at the GGNB program Microbiology and Biochemistry

Georg August University of Göttingen (Germany)

Title of thesis: "A possible functional link between RNA degradation and transcription in Bacillus subtilis"

Supervisor: Prof. Dr. Jörg Stülke

- 2014 - 2016: Master studies in Genetics, Molecular Biology and Virology

Charles University in Prague (Czech Republic), Faculty of Science

Specialization: Molecular Biology and Genetics of Prokaryotes

Title of thesis: Transcription regulation by sigma factors in Bacillus subtilis

Supervisor: Libor Krásný PhD.

- 2011 - 2014: Bachelor studies in Molecular Biology and Biochemistry of Organisms Charles University in Prague (Czech Republic), Faculty of Science

Title of thesis: Regulation of bacterial transcription by alternative sigma factors Supervisor: Libor Krásný PhD. 